Chapter 18

\title{
Prediction of the Stability of Unsteady Motions in Solid-Propellant Rocket Motors
}

\author{
F. E. C. Culick* \\ California Institute of Technology, Pasadena, California \\ V. Ÿangt \\ Pennsylvania State University, University Park, Pennsylvania
}

\section{Nomenclature}

$a \quad$ = speed of sound, Eq. (23)

$A_{b}=$ defined in Eq. (92b)

$A_{n}=$ defined in Eq. (58)

$A_{n i j}=$ defined in Eq. (75)

$B_{n}=$ defined in Eq. (58)

$B_{n i j}=$ defined in Eq. (75)

$C=$ specific heat of particle material

$C_{m}=$ mass fraction of particles, $C_{m}=\rho_{p} / \rho_{g}$

$\bar{C}_{P}=$ mass-averaged specific heat, Eq. $(20)$

$\bar{C}_{v}=$ mass-averaged specific heat, Eq. (20)

$D_{n i}=$ defined in Eq. (75)

$e_{0}=$ stagnation energy, gas phase

$E_{n}^{2}=$ defined in $\mathrm{Eq}$. (47b)

$E_{n i}=$ defined in Eq. (75)

$\langle\mathscr{E}\rangle=$ time-averaged energy

$f=$ defined in Eq. (42)

$f_{1}=$ defined in Eq. (52b)

Copyright $\mathbb{C} 1990$ by F. E. C. Culick and V. Yang. Published by the American Institute of Aeronautics and Astronautics, Inc, with permission.

${ }^{*}$ Professor of Mechanical Engineering and Jet Propulsion.

†Associate Professor of Mechanical Engineering. 
$F_{n}=$ defined in Eq. (49)

$F_{p}=$ force of interaction, Eq. (13)

$\delta F_{p}=$ defined in Eq. (18a)

$\mathscr{F}^{p}=$ defined in Eq. (28)

$\mathscr{F}_{1 s}=$ defined in Eq. (36b)

$h=$ defined in Eq. (40)

$h_{1}=$ defined in Eq. (52a)

$k=$ complex wave number, $(\omega-i \alpha) / \bar{a}$

$k_{t}=$ wave number, $w_{\ell} / a$

$K=$ defined in Eq. (142)

$m_{b}^{(g)}=$ mass flux of gas inward

$m_{b}^{(p)}=$ mass flux of particles inward

$\bar{M}_{b}=$ average Mach number of flow at the propellant surface

$p=$ pressure

$\mathscr{P}=$ defined in Eq. (29)

$\mathscr{P}_{1 s}=$ defined in Eq. (36c)

$Q=$ energy addition (energy/vol-s)

$Q_{p}=$ energy transfer, Eq. (14)

$\delta Q_{p}=$ defined in Eq. (18b)

$\bar{R}^{p}=$ mass-averaged gas constant, Eq.

$R_{b}=$ defined in Eq. (92a)

$r_{n}=$ defined in Eq. (58)

$S_{c}=$ cross-sectional area

$T=$ temperature

$\Delta \hat{T}_{s}=$ defined in Eq. (90)

$u \quad=$, velocity

$w_{p}=$ "mass source (mass/vol-s), Eq. (7)

$\mathscr{W}=$ defined in Eq. (27)

$\mathcal{W}_{1 s}=$ defined in Eq. (36a)

$X_{1}=$ defined in Eq. (124a)

$X_{2}=$ defined in Eq (124b)

$\alpha=$ growth constant

$\alpha_{c}=$ defined in Eq. (99)

$\alpha_{F T}=$ defined in Eq. (151)

$\alpha_{N}=$ defined in Eq. (100)

$\alpha_{P}=$ defined in Eq. (125a)

$\bar{\gamma}=$ ratio of specific heats

$\eta_{n}=$ time-dependent amplitude, Eq. (58)

$\kappa_{p}=$ thermal diffusivity of propellant

$\lambda=$ defined in Eq. (95)

$\mu \quad=$ viscosity

$\bar{v} \quad=$ kinematic viscosity

$\rho=$ density (mass/vol)

$\vec{\sigma} \quad=$ defined in Eq. (15)

$\sigma=$ particle diameter

$\tau_{d}=$ defined in Eq. (122a)

$\tau_{t}=$ defined in Eq. (122b)

$\phi_{n}=$ defined in Eq. (58) 
$\psi_{n}=$ classical mode shape

$\omega=$ frequency

$\omega_{n}=$ classical acoustic frequency

\author{
Subscripts \\ $g \quad$ = gas properties \\ $p \quad=$ particle properties \\ $1=$ one-dimensional values \\ $s \quad=$ evaluated at the burning surface
}

Superscripts

$\left(^{-}\right)$mass-averaged or time-averaged property

()$^{\prime}=$ fluctuation

(^) = complex amplitude

\title{
I. Introduction
}

THE phenomena called "combustion instabilities" in a solid rocket motor may be viewed as the unsteady motions of a dynamic system capable of sustaining oscillations over a broad range of frequencies. Energy is supplied by combustion processes confined chiefly to thin regions near the surface of the burning propellant. Interactions between the unsteady motions and the burning convert a small fraction of the heat released by combustion to potential and kinetic energy of the gases within the chamber.

It is somewhat misleading that the term combustion instability has become standard. The presence of an instability in any combustion chamber is established by observing either the behavior of the gas pressure or accelerations of the enclosure, and occasionally both. Combustion itself is not unstable except possibly in some cases when explosion ensues or in some instances of extinction. An instability arises because of the coupling between the burning processes and the gasdynamic motions, both of which alone may be stable.

An observer perceives an unstable motion as "self-excited": the amplitude of the pressure fluctuation grows out of the noise without benefit of any external influence. Hence, the theory of these phenomena belongs to the general theory of self-excited dynamic systems. The prevalence of instabilities in combustion systems is due primarily to two fundamental causes:

1) The energy required to generate an unacceptably large amplitude of unsteady motion is a negligibly small part of the energy available and released in combustion of the reactants.

2) Combustion chambers are almost entirely closed, and the internal processes tending to attenuate unsteady motions are weak.

These causes are present in any combustion chamber but are especially consequential for solid-propellant rockets in which the energy release per unit volume (or surface area) is extremely high. Two conclusions are im- 
mediate: 1) the possibility of instabilities must be recognized and anticipated from the beginning of a development program, and 2) the occurrence of an instability in a motor is not necessarily the result of stupidity or of maladroit design procedures. Occurrence of the problem must be regarded as part of the price for the development of a new system.

It is therefore essential that theory be constructed to provide a basis for analyzing and predicting unsteady motions in combustion chambers. The phenomena to be treated are extremely complicated, involving nonlinear gasdynamics and the combustion of chemical systems that cannot theoretically be described in all necessary detail. Hence, we must be guided at all stages by observational results. The theory is substantially a vehicle for organizing and interpreting experimental results. It is not possible to predict accurately the occurrence of instabilities from first principles. Nevertheless, with judicious melding of theory, measurements, and observations, we can construct a framework that provides the basis both for understanding the general behavior and for formulating simple statements succinctly summarizing the general characteristics. For practical purposes, achievements of the theory consist mainly in the ability to analyze, understand, and predict trends of behavior. The influences of geometric characteristics and combustion processes can be assessed but, in any event, some experimental data are required to provide quantitative results.

We may distinguish two sorts of theoretical work: analytical and numerical. By "numerical" we mean results obtained by numerical solution of the partial differential equations governing the behavior of the system. At present, it is not possible to progress with exact formal analysis of those equations; "analytical" therefore implies some sort of approximate method.

Numerical methods have the disadvantage that, even when dimensionless variables are used, only restricted results can be obtained. It is therefore tedious and expensive to discover general trends of behavior or summarize qualitative features. On the other hand, approximate analytical methods carry with them inaccuracies that cannot usually be estimated with confidence. Because of the semiempirical nature of the theory, comparison with experimental results does not afford a sufficient basis for confirming the validity of any approximations. Thus, an important function of numerical methods is to provide a means for checking the accuracy of approximate methods. This strategy works only if the methods being compared are applied to the same problems. Care must be exercised because differences in the formulations (approximate or numerical) often do not permit a precise correspondence between the required input data and, possibly, the boundary conditions as well.

In this chapter, we discuss both approaches. To provide background and motivation for the construction of the approximate analysis, we begin in $\mathrm{Sec}$. II with a brief description of the important features of combustion instabilities in solid rocket motors. The equations of conservation, the common basis for all numerical and analytical work, are discussed in Sec. III, followed by application to problems of linear stability in Sec. IV. Some aspects of nonlinear behavior are covered in Sec. V. Section VI is a short review of numerical calculations of some one-dimensional problems. 


\section{Some General Features of Combustion Instabilities in Solid- Propellant Rocket Motors}

Combustion instabilities in different systems are distinguished primarily by geometry and the manner in which reactants are introduced. In any case, the presence of an instability in a chamber has always been identified with the presence of unsteady motions having quite well-defined frequencies. From the earliest observations to the present, the frequencies have usually been close to those computed for the classical acoustic modes of the chamber if the boundary condition at the nozzle is suitably approximated. The physical reason for this behavior is that the combustion processes and mean flow are relatively small perturbations of the classical acoustic behavior, even though they are the necessary causes for the instabilities. That result is the basis for the form of the theory developed in this chapter.

We may estimate the frequencies by ignoring the combustion processes and the mean flow and considering a chamber having the same shape as the volume containing the combustion gases but entirely enclosed by a rigid boundary. The exit boundary is closed at the entrance to the exhaust nozzle. That may be somewhat ambiguous, particularly for a re-entrant nozzle, but an approximate choice is good enough for the purposes here. Then the chamber is assumed to contain gases having the same thermodynamic properties as the actual combustion products. It is especially important that the presence of condensed material be taken into account. Then the speed of sound is known; its value and the geometry of the equivalent closed chamber determine the frequencies and mode shapes for the classical acoustic modes.

The simplest example is a chamber of length $L$ having uniform cross section. No matter what the shape of the cross section, the axial or longitudinal modes are the same as those for a closed-closed organ pipe. This characterization is applied because the velocity fluctuations are longitudinal, parallel to the axis, and, in this idealized limit, uniform over a cross section. The velocity must vanish at the ends and varies sinusoidally along the axis, being proportional to $\sin k_{\ell} z$. Here $k_{\ell}$ is the wave number for the $\ell$ th mode, defined in terms of the angular frequency $\omega_{\ell}$ and the speed of sound $a$ as

$$
k_{\ell}=\frac{\omega_{\ell}}{a}
$$

Classical theory shows that the eigenvalues $k_{\ell}$ are

$$
k_{\ell}=\ell \frac{\pi}{L}
$$

and the cyclic frequencies are

$$
f_{\ell}=\frac{\omega_{\ell}}{2 \pi}=\ell \frac{a}{2 L}
$$


Thus, for purely longitudinal modes, the modal frequencies are integral multiples of the fundamental frequency $f_{1}=a / 2 L$.

Because it is directly observable, the pressure is a convenient property for characterizing instabilities. The acoustic pressure attains maximum value at those regions in the chamber in which the velocity is minimum. Hence, the pressure varies as $\cos k_{\ell} z$, having greatest values at the ends. It is usually true that, whereas the pressure fluctuations may have local maxima in the interior of the chamber, there are maxima, or antinodes, at the boundary. Thus, by analogy with Eq. (3), the frequency for the fundamental mode of oscillations in transverse planes is roughly $a / 2 D$, where $D$ is the diameter of the chamber. Typically, then, the frequencies of axial modes are lower than those of transverse modes by the ratio $D / L, D$ being less than $L$ for most solid rocket motors.

The physical basis for these estimates is the fundamental result for a wave motion that the frequency $f$, speed of propagation $a$, and wavelength $\lambda$ are related by $f \lambda=a$. What we are really doing is estimating the wavelength, which is determined by the geometry and the boundary condition. For motions between two rigid surfaces where the perpendicular acoustic velocity must vanish, an integral number of half-waves will fit. Thus, the wavelength of the fundamental mode (having the greatest wavelength) is twice the distance between the surfaces, $2 L$ or $2 D$ in the example given earlier. This procedure works for a solid rocket because the flow speeds are usually small compared with the speed of sound, and the acoustic velocity perpendicular to the boundary is indeed nearly zero. However, we note that there are exceptional cases, notably one in which the Mach number is large at the entrance to the exhaust nozzle.

Let $\delta p$ denote the maximum amplitude of oscillation in a longitudinal mode, so that the acoustic pressure is

$$
p=\delta p \cos k_{\rho} z
$$

The instantaneous energy $\mathscr{E}$ in the mode is the sum of the potential energy, proportional to $p^{2}$; and the kinetic energy, proportional to $u^{2}$. Because the acoustic velocity and pressure are related, the time-averaged energy $\langle\mathscr{E}\rangle$ can be expressed in terms of the pressure only and is given by the formula

$$
\langle\mathscr{E}\rangle=\frac{\bar{p} L S_{c}}{4 \gamma}\left(\frac{\delta p}{\bar{p}}\right)^{2}
$$

where $\bar{p}$ is the mean pressure. Consider a motor having length $L=1 \mathrm{~m}$ and a cross-sectional area $S_{c}=0.02 \mathrm{~m}^{2}$ and operating at a mean pressure of $100 \mathrm{~atm}\left(1 \times 10^{7} \mathrm{~N} / \mathrm{m}^{2}\right)$. For $\gamma=1.2$, the last formula gives $\langle\mathscr{E}\rangle=$ $41,666(\delta p / \bar{p})^{2} \mathrm{~J}$. If the amplitude $\delta p$ is $10 \%$ of the mean pressure, $\langle\mathscr{E}\rangle \cong$ $400 \mathrm{~J}$. This may be compared to a representative value of the heat of reaction, say, $4 \times 10^{6} \mathrm{~J} / \mathrm{kg}$ of propellant.

Thus, the energy in the acoustic field, even when the amplitude becomes comparable to the mean pressure, is a negligible fraction of the total energy available in the system. That is one reason why, as noted earlier, instabilities are likely events in solid rocket motors. This result, however, bears 
little relation to the matter of stability: we need an estimate of the rate at which energy is gained by the acoustic waves, and we need to compare that with the rate at which energy is actually made available by the combustion processes.

When a steady oscillation is present in a chamber, the rate at which acoustic energy is lost must precisely balance the rate at which energy is transferred to the waves from the combustion processes. If these rates differ, then the amplitude of the motion will grow or decay. Consider a wave that is growing, and suppose that the behavior is that of an unstable linear system. Then the amplitude of the pressure fluctuation is proportional to $\exp (\alpha t)$, where $\alpha$ is the growth constant; but the energy is proportional to $(\delta p)^{2}$ and therefore varies as $\exp (2 \alpha t)$. Hence, approximately, the time-averaged energy has the behavior $\langle\mathscr{E}\rangle \sim \exp (2 \alpha t)$, and its rate of change is

$$
\frac{\mathrm{d}\langle\mathscr{E}\rangle}{\mathrm{d} t}=2 \alpha\langle\mathscr{E}\rangle
$$

This relation is valid if nonlinear processes are negligible and providing that $\alpha$ is much less than the frequency $(\alpha<<\omega)$. For the chamber considered earlier and for $a=1200 \mathrm{~m} / \mathrm{s}$, Eq. (3) gives $f_{1}=600 \mathrm{~Hz}$. In practice, $\alpha$ is usually less than $100 \mathrm{~s}^{-1}$ and, for a frequency of $600 \mathrm{~Hz}, \omega \approx 3800$ $\mathrm{rad} / \mathrm{s}$. Hence, $\alpha<<\omega$. Then, with $\delta p / \bar{p}=0.1$ and $\langle\mathscr{E}\rangle=400 \mathrm{~J}$, the acoustic energy changes at the rate $8 \times 10^{4} \mathrm{~J} / \mathrm{s}$.

Because the growth rate is, in fact, the rate at which energy is gained from the combustion processes minus the loss rate due to damping mechanisms, the result just given underestimates the power provided by unsteady combustion. Typically, the net growth rate is the difference between two relatively larger numbers. In extreme cases, when large damping is present, the rate of energy supplied by the burning may be 5-10 times as large as the net rate at which the acoustic field gains energy. When this occurs, substantial increases in the average burning rate, chamber pressure and, therefore, thrust may be observed.

The rate at which energy is released by combustion is the rate at which solid material is consumed, multiplied by the heat of reaction $H, \rho_{s} r S_{b} H$, where $\rho_{s}$ is the density of the solid, $r$ the linear burning rate, and $S_{b}$ the area of burning surface. For $\rho_{s} \approx 1600 \mathrm{~kg} / \mathrm{m}^{3}, r=0.75 \mathrm{~cm} / \mathrm{s}, S_{b} \approx 0.5 \mathrm{~m}^{2}$ for a circular cross section having area $0.02 \mathrm{~m}^{2}$, and $H=4 \times 10^{6} \mathrm{~J} / \mathrm{kg}$, we find $\rho_{s} r S_{b} H=2.4 \times 10^{7} \mathrm{~J} / \mathrm{s}$. Obviously, only a very small portion of this, less than $0.4 \%$, is required to drive the acoustic waves at the rate estimated here. This striking result shows that we might expect acoustic waves to grow in a chamber with essentially no influence on the steadystate performance unless unstable waves are driven in a highly damped system. Whether even the small rates of energy transfer commonly required to create an instability will actually be achieved depends on the interactions between the waves and the oscillations. Only weak coupling is required, and it is fortunate that, in practice, the interactions are as ineffective as they are; the potential is always present for catastrophic results.

Another source of energy for unsteady motions is the mean flowfield itself and fluctuations associated, for example, with flow separation. There 
are many examples of acoustic waves excited by these causes, both in laboratory devices and in motors. Experience has confirmed that, in those cases as well, the interactions responsible for the unstable motions are weak, and the amplitudes of the waves do not reach large amplitudes, although they may cause troublesome vibrations of the vehicle.

We conclude that, for the theoretical purposes, we may regard combustion instabilities as unsteady motions that are, in some sense, closely related to the classical acoustic modes. Perturbations of the acoustic modes are associated primarily with the combustion processes and the mean flow. The theoretical representation of instabilities conforms with this physical behavior. Specifically, the approximate analysis discussed later is founded on construction of a wave equation and boundary conditions having source terms arising from the various perturbations. The basic motions are the linear acoustic waves easily determined from classical acoustic theory.

The source terms may be divided into two kinds: those that depend linearly on the fluctuating motion and those that are nonlinear. Linear contributions are responsible for unstable motions. If conditions produce an unstable wave, the amplitude grows exponentially in time. Because the system is self-excited, a limiting amplitude is reached - the system executes a periodic limit cycle-only if nonlinear processes are active. Thus, we may arrive at a full understanding of the phenomena only if we treat nonlinear behavior.

A special advantage of the approximate analysis is that it displays explicitly the contributions of all physical processes. The results provide a clear framework for interpreting and predicting instabilities quantitatively. Moreover, the form of the results suggests the sorts of experiments we should perform to obtain the information necessary to allow analysis of practical systems.

Purely numerical analysis also reproduces the physical behavior just described. However, it is then not easy to distinguish in detail the influences of the various processes acting. In principle, numerical methods may be applied to any system and any geometry but, in fact, even with recent advances in computing, the computations remain difficult and expensive. To date, results have been obtained chiefly for one-dimensional problems, with some limited treatment of two-dimensional problems. Some aspects of unsteady flows are better treated with numerical methods, even though the results are necessarily limited to particular cases. Later we shall examine some one-dimensional calculations to illustrate comparison with approximate results. Nevertheless, the numerical approach unquestionably has an important place in the analysis of practical systems and will no doubt be used increasingly as the cost of large-scale calculations continues to decrease.

\section{Equations of Motion and Formulation of the Analysis}

Analysis of flows in solid-propellant rockets must be based on the equations of conservation for a gas containing condensed species. Because of uncertainties in the actual flow properties, it is inappropriate to use a completely general formulation. We assume here a perfect gas and one condensed species present as small particles. The complete set of conser- 
vation equations then comprises those for the gas and those for the particulate material. We may combine the equations to form a set governing the motions of a single medium as, for example, discussed by Marble $\mathrm{e}^{1,2}$ in his papers on two-phase flows. Application of that idea to the sorts of problems treated here was developed by Culick. ${ }^{3}$

\section{A. Conservation Equations for a Two-Phase Flow}

Residual combustion of both the gas-phase and condensed material may occur within the volume. The first is accounted for as a heat source; the second may also involve a conversion of condensed mass to gas, denoted here as $w_{p}$ (mass/time-volume). We also assume, which is not quite correct, that the gases produced by the burned condensed material have the same velocity as their sources $\left(\boldsymbol{u}_{p}\right)$. For simplicity, we ignore viscous stresses and heat conduction in the gas phase, an approximation that can be easily improved. The conservation equations are

Mass (gas):

$$
\frac{\partial \rho_{g}}{\partial t}+\nabla \cdot\left(\rho_{g} u\right)=w_{p}
$$

Mass (particles):

$$
\frac{\partial \rho_{p}}{\partial t}+\nabla \cdot\left(\rho_{p} \boldsymbol{u}_{p}\right)=-w_{p}
$$

Momentum:

$$
\frac{\partial}{\partial t}\left(\rho_{g} \boldsymbol{u}+\rho_{p} \boldsymbol{u}_{p}\right)+\nabla \cdot\left(\rho_{g} \boldsymbol{u} \boldsymbol{u}+\rho_{p} \boldsymbol{u}_{p} \boldsymbol{u}_{p}\right)+\nabla p=0
$$

Energy:

$$
\frac{\partial}{\partial t}\left(\rho_{g} e_{0}+\rho_{p} e_{p 0}\right)+\nabla \cdot\left(\rho_{g} \boldsymbol{u} e_{0}+\rho_{p} u_{p} e_{p 0}\right)+\nabla \cdot(p \boldsymbol{u})=Q
$$

Energy released in homogeneous reactions in the gas phase is represented by $Q$ (energy/time-volume). For simplicity, we ignore the influences of viscous stresses and heat transfer internal to the gas, processes that usually have negligible effects in the problems we treat here. These can be accommodated within the approximate analysis, appearing as additional source terms in Eqs. (8) and (10).

With simple manipulations, we can write the momentum and energy equations as

$$
\begin{gathered}
\rho_{g} \frac{\partial \boldsymbol{u}}{\partial t}+\rho_{g} \boldsymbol{u} \cdot \nabla \boldsymbol{u}+\nabla p=\boldsymbol{F}_{p}-\boldsymbol{\sigma} \\
\rho_{g} C_{\nu} \frac{\partial T}{\partial t}+\rho_{g} C_{\nu} \boldsymbol{u} \cdot \nabla T+p \nabla \cdot \boldsymbol{u}=Q+Q_{p}+\left(e_{p 0}-e_{0}\right) w_{p} \\
+\boldsymbol{u} \cdot \boldsymbol{\sigma}+\left(\boldsymbol{u}_{p}-\boldsymbol{u}\right) \cdot \boldsymbol{F}_{p}
\end{gathered}
$$


The force of interaction between the gas and particles is

$$
\boldsymbol{F}_{p}=-\rho_{p}\left[\frac{\partial \boldsymbol{u}_{p}}{\partial t}+u_{p} \cdot \nabla \boldsymbol{u}_{p}\right]
$$

and the heat release associated with chemical reactions and transfer of heat between the condensed and gaseous phase is

$$
Q_{p}=-\rho_{p} C\left[\frac{\partial T_{p}}{\partial t}+u_{p} \cdot \nabla T_{p}\right]
$$

The momentum transfer to the gas associated with residual combustion is

$$
\boldsymbol{\sigma}=\left(\boldsymbol{u}-\boldsymbol{u}_{p}\right) w_{p}
$$

It is important that the correct speed of sound appears to zeroth order in the wave equation deduced later, namely, the value for conditions near equilibrium for the gas/particle mixture. The manipulations carried out to insure that result are discussed by Culick. ${ }^{3}$ As a result, the momentum and energy equations appropriate to the propagation of disturbances in the mixture are

$$
\begin{gathered}
\rho \frac{\partial \boldsymbol{u}}{\partial t}+\rho \boldsymbol{u} \cdot \nabla \boldsymbol{u}+\nabla p=\delta \boldsymbol{F}_{p}-\boldsymbol{\sigma} \\
\rho \bar{C}_{v}\left(\frac{\partial T}{\partial t}+\boldsymbol{u} \cdot \nabla T\right)+p \nabla \cdot \boldsymbol{u}=Q+\delta Q_{p}+\left(e_{p 0}-e_{0}\right) w_{p} \\
+\boldsymbol{u} \cdot \boldsymbol{\sigma}+\delta \boldsymbol{u}_{p} \cdot \boldsymbol{F}_{p}
\end{gathered}
$$

where

$$
\begin{array}{r}
\delta F_{p}=-\rho_{p}\left[\frac{\partial \delta u_{p}}{\partial t}+\delta u_{p} \cdot \nabla \delta u_{p}+\delta u_{p} \cdot \nabla u+u \cdot \nabla \delta u_{p}\right] \\
\delta Q_{p}=-\rho_{p} C\left[\frac{\partial \delta T_{p}}{\partial t}+\delta u_{p} \cdot \nabla \delta T_{p}+\delta u_{p} \cdot \nabla T+\boldsymbol{u} \cdot \nabla \delta T_{p}\right]
\end{array}
$$

and $\delta u_{p}=u_{p}-\boldsymbol{u}, \delta T_{p}=T_{p}-T$. The density $\rho$ for the mixture is

$$
\rho=\rho_{g}+\rho_{p}=\rho_{g}\left(1+C_{m}\right)
$$

and $C_{m}=\rho_{p} / \rho_{g}$ is the mass fraction of particles.

Strictly, $C_{m}$ must be treated as a dependent variable. Any nonuniformity in the mean flowfield, or unsteady motions, will cause the particles to "slip" relative to the gas. Not only will $\delta u_{p}$ and $\delta T_{p}$ be nonzero, but also the temperature within an individual particle will not be uniform. A complete treatment of the flow requires that the particle velocity and temperature be calculated as functions of time and position within the chamber. That procedure requires solutions to Eq. (13) and (14), with the force of 
interaction and the heat transfer specified. Simultaneously, the temperature field within the particle should be determined, an analysis rendered even more difficult for vaporizing and burning particles. Such matters are subjects of current research in the field of spray combustion and will not be discussed here. (See, for example, Refs. 4-6.)

In the limit of infinitesimally small particles, the gas and particles are in equilibrium, in the sense that the local temperatures and velocities are equal: $T=T_{p} ; \boldsymbol{u}=\boldsymbol{u}_{p}$. Then the particles follow the gas motion and, if the initial distribution of the particles is uniform, the $C_{m}$ is constant throughout the chamber. The assumption $C_{m}$ constant is normally made in analysis of the flows in solid-propellant rockets, and we shall follow that practice here. Although errors incurred can be estimated for typical flow conditions, nothing is to be gained by doing so here. The influences of unsteady motions and residual combustion can be accommodated within the approximate analysis, but the necessary calculations have not been done.

The mass-weighted specific heats for the mixture are defined in the usual fashion $^{1}$ :

$$
\bar{C}_{v}=\frac{C_{v}+C_{m} C}{1+C_{m}}, \quad \bar{C}_{p}=\frac{C_{p}+C_{m} C}{1+C_{m}}
$$

Now, add $T$ times Eqs. (7) and (8) to $1 / \bar{C}_{\nu}$ times Eq. (17) to find an equation for the pressure:

$$
\begin{aligned}
& \frac{\partial p}{\partial t}+\boldsymbol{u} \cdot \nabla p+\bar{\gamma} p \nabla \cdot \boldsymbol{u}=\frac{\bar{R}}{\bar{C}_{v}}\left[Q+\delta Q_{p}+\delta \boldsymbol{u}_{p} \cdot \boldsymbol{F}_{p}+\boldsymbol{u} \cdot \boldsymbol{\sigma}\right. \\
& \left.\quad+\left(e_{p 0}-e_{0}\right) w_{p}-\bar{C}_{v} T \nabla \cdot\left(\rho_{p} \delta \boldsymbol{u}_{p}\right)\right]
\end{aligned}
$$

The perfect-gas law, $p=\rho_{g} R T$, has been used, where $R$ is the gas constant for the gas only. For the mixture, $\bar{R}=\bar{C}_{p}-\bar{C}_{v}, \bar{\gamma}=\bar{C}_{p} / \bar{C}_{\nu}$, and

$$
p=\rho \bar{R} T
$$

The chief purpose of the preceding exercise is to establish the forms of the equations that account for the presence of condensed material and that will provide the most accurate value for the speed of sound for the unperturbed motions, namely,

$$
a=(\bar{\gamma} \bar{R} T)^{1 / 2}=\left[\frac{\bar{\gamma}}{1+C_{m}}\left(\frac{p}{\rho_{g}}\right)\right]^{1 / 2}
$$

The right-hand sides of the reformed conservation equations, in particular, Eqs. (16) and (21), contain the major contributions causing combustion instabilities. We are less concerned here with the details than with the construction of a general framework. Hence, to simplify writing, we represent the source terms in general form and write the equations for conservation of mass, momentum, and energy (written in terms of the pressure) 
as

$$
\begin{aligned}
\frac{\partial \rho}{\partial t}+\boldsymbol{u} \cdot \nabla \rho & =\mathscr{W} \\
\rho \frac{\partial u}{\partial t}+\rho \boldsymbol{u} \cdot \nabla u & =-\nabla p+\mathscr{F} \\
\frac{\partial p}{\partial t}+\bar{\gamma} p \nabla \cdot u & =-\boldsymbol{u} \cdot \nabla p+\mathscr{P}
\end{aligned}
$$

For the circumstances treated earlier,

$$
\begin{gathered}
\mathscr{W}=-\rho \nabla \cdot u-\nabla \cdot\left(\rho_{p} \delta \boldsymbol{u}_{p}\right) \\
\mathscr{F}=\delta \boldsymbol{F}_{p}+\delta \boldsymbol{u}_{p} w_{p} \\
\mathscr{P}=\frac{\bar{R}}{\bar{C}_{\nu}}\left[Q+\delta Q_{p}+\delta \boldsymbol{u}_{p} \cdot \boldsymbol{F}_{p}+\left\{\left(e_{p}-e\right)+\frac{1}{2}\left(\delta \boldsymbol{u}_{p}\right)^{2}\right\} w_{p}\right. \\
\left.-\bar{C}_{\boldsymbol{v}} T \nabla \cdot\left(\rho_{p} \delta \boldsymbol{u}_{p}\right)\right]
\end{gathered}
$$

Equations (24-26) are suitable for two- and three-dimensional problems. Because of the sources of mass, momentum, and energy at the lateral boundaries of solid rockets, treatment of problems within the one-dimensional approximation requires special attention. In effect, some of the processes that arise in the boundary conditions placed on these equations appear explicitly as source terms in the one-dimensional equations. The formulation was discussed first by Culick. ${ }^{7,8}$ For a chamber having a nonuniform cross-sectional area $S_{c}(z)$, the equations corresponding to Eqs. $(24-26)$ are

$$
\begin{gathered}
\frac{\partial \rho}{\partial t}+u \frac{\partial \rho}{\partial z}=\mathscr{W}_{1} \\
\rho \frac{\partial u}{\partial t}+\rho u \frac{\partial u}{\partial z}=-\frac{\partial p}{\partial z}+\mathscr{F}_{1} \\
\frac{\partial p}{\partial t}+\bar{\gamma} p \frac{1}{S_{c}} \frac{\partial}{\partial z}\left(u S_{c}\right)=-u \frac{\partial p}{\partial z}+\mathscr{P}_{1}
\end{gathered}
$$

where

$$
\begin{gathered}
\mathscr{W}_{1}=-\rho \frac{1}{S_{c}} \frac{\partial}{\partial z}\left(u S_{c}\right)-\frac{1}{S_{c}} \frac{\partial}{\partial z}\left(S_{c} \rho_{p} \delta u_{p}\right)+W_{1 s} \\
\mathscr{F}_{1}=\delta F_{p}+\delta u_{p} w_{p}+\mathscr{F}_{1 s} \\
\delta w_{p}=w_{p}-\mathcal{W}_{p}
\end{gathered}
$$




$$
\begin{aligned}
\mathscr{P}_{1} & =\frac{\bar{R}}{\bar{C}_{\nu}}\left[Q+\delta Q_{p}+\delta u_{p} F_{p}+\left\{\left(e_{p}-e\right)+\frac{1}{2}\left(\delta u_{p}\right)^{2}\right\} w_{p}\right. \\
& \left.-\bar{C}_{v} T \frac{1}{S_{c}} \frac{\partial}{\partial z}\left(S_{c} \rho_{p} \delta u_{p}\right)\right]+\mathscr{P}_{1 s}
\end{aligned}
$$

where

$$
\begin{aligned}
\mathscr{W}_{1 s} & =\frac{1}{S_{c}} \int m_{b} \mathrm{~d} q \\
\mathscr{F}_{1 s} & =\frac{1}{S_{c}} \int\left[\left(u_{s}-u\right) m_{b}^{(g)}+\left(u_{p s}-u_{p}\right) m_{b}^{(p)}\right] \mathrm{d} q \\
\mathscr{P}_{1 s} & =\frac{\bar{R}}{\bar{C}_{v}} \frac{1}{S_{c}} \int\left[\left(h_{0 s}-e_{0}\right) m_{b}^{(g)}+\left(e_{p 0 s}-e_{p 0}\right) m_{b}^{(p)}+\bar{C}_{\nu} T m_{b}^{(g)}\right] \mathrm{d} q \\
& +\frac{\bar{R}}{C_{v}} \frac{u}{S_{c}} \int\left[\left(u-u_{s}\right) m_{b}^{(g)}+\left(u_{p}-u_{p s}\right) m_{b}^{(p)}\right] \mathrm{d} q
\end{aligned}
$$

The terms containing integrals over the perimeter $q$ represent the influences of material entering the chamber at the lateral boundary. Properties of the injected flow are denoted by subscript $s$, except that $m_{b}^{(g)}$ and $m_{b}^{(p)}$ represent the mass flux of gas and particles respectively, normal to the boundary, with $m_{b}=m_{b}^{(g)}+m_{b}^{(p)}$ the total mass flux.

Note that, apart from the additional source terms at the lateral boundary, the one-dimensional equations may be deduced directly from the threedimensional equations [Eqs. (24-26)]. Replace $\boldsymbol{u}$ by $\boldsymbol{u}, \boldsymbol{u}_{p}$ by $\boldsymbol{u}_{p}, \boldsymbol{u} \cdot \nabla$ by $u(\partial / \partial z)$, and $\nabla \cdot()$ by $\left(1 / S_{c}\right)(\partial / \partial z)\left(S_{c}\right)$.

When particulate material is present in the flow, the properties $\boldsymbol{u}_{p}$ (or $u_{p}$ ) and $T_{p}$ must be determined by solving Eqs. (13) and (14). The average force $F_{p}$ and heat transfer $Q_{p}$ must then be specified. Those processes depend on $\delta u_{p}$ and $\delta T_{p}$, coupling the particle and gas motions. Later we shall discuss that problem, showing how to obtain elementary results for the attenuation of unsteady motions from gas/particle interactions.

\section{B. Formulation of the Approximate Analysis for Second-Order Acoustics: Spatial and Time Averaging}

The sets of equations, Eqs. (24-26) and (30-32), may be used as the basis for numerical analysis of unsteady flows. Here we are concerned with construction of an approximate analysis, which we develop for the case of three-dimensional motions. The general results can readily be specialized to one-dimensional problems, with proper attention to the correct forms of the source terms $\mathscr{W}_{1}, \mathscr{F}_{1}$, and $\mathscr{P}_{1}$.

To avoid preoccupation with details unnecessary at this stage, we shall not display explicitly the contributions to $\mathscr{W}, \mathscr{F}$, and $\mathscr{P}$. All dependent variables are written as sums of mean and fluctuating parts, $p=\bar{p}+p^{\prime}$, etc.; to simplify the derivation, we assume that the average values do not 
vary with time. That is not an essential assumption, but to correct it requires considerable elaboration not justified here. However, there are practical situations in which changes in the average values, particularly the pressure, are important. No thorough analysis of such cases has been given.

Written to second order in fluctuations, Eqs. (25) and (26) become

$$
\begin{aligned}
& \bar{\rho} \frac{\partial \boldsymbol{u}^{\prime}}{\partial t}+\nabla p^{\prime}=-\bar{\rho}\left(\overline{\boldsymbol{u}} \cdot \nabla \boldsymbol{u}^{\prime}+\boldsymbol{u}^{\prime} \cdot \nabla \overline{\boldsymbol{u}}\right) \\
& -\bar{\rho}\left(\boldsymbol{u}^{\prime} \cdot \nabla \boldsymbol{u}^{\prime}\right)-\rho^{\prime} \frac{\partial \boldsymbol{u}^{\prime}}{\partial t}+\mathscr{F}{ }^{\prime} \\
& \frac{\partial p^{\prime}}{\partial t}+\bar{\gamma} \bar{p} \nabla \cdot \boldsymbol{u}^{\prime}=-\overline{\boldsymbol{u}} \cdot \nabla p^{\prime}-\bar{\gamma} p^{\prime} \nabla \cdot \overline{\boldsymbol{u}} \\
& -\boldsymbol{u}^{\prime} \cdot \nabla p^{\prime}-\bar{\gamma} p^{\prime} \nabla \cdot \boldsymbol{u}^{\prime}+\mathscr{P}
\end{aligned}
$$

The mean velocity varies within the chamber, but the average pressure is assumed constant. That amounts to assuming that the average Mach number is small, and so terms of order $|\overline{\boldsymbol{u}}|^{2}\left|\boldsymbol{u}^{\prime}\right|$ and $|\overline{\boldsymbol{u}}|\left|\boldsymbol{u}^{\prime}\right|^{2}$ are neglected.

Equations (37) and (38) are, respectively, a vector and a scalar equation for the velocity and the pressure fluctuations. The density and temperature fluctuations are also dependent variables, and so the system of equations is completed by adding the equation for $\rho$ ' derived from the conservation of mass [Eq. (24)] and the perturbed form of the equation of state [Eq. (22)]. Those equations will not be written at this point but will be introduced when required.

Now, differentiate Eq. (38) with respect to time, and substitute Eq. (37) for $\partial u^{\prime} / \partial t$ to find the nonlinear wave equation:

$$
\nabla^{2} p^{\prime}-\frac{1}{\bar{a}^{2}} \frac{\partial^{2} p^{\prime}}{\partial t^{2}}=h
$$

where $\bar{a}^{2}=\bar{\gamma} \bar{R} \bar{T}$ is independent of position, and

$$
\begin{aligned}
h & =-\bar{\rho} \nabla \cdot\left(\bar{u} \cdot \nabla \boldsymbol{u}^{\prime}+\boldsymbol{u}^{\prime} \cdot \nabla \bar{u}\right)+\frac{1}{\bar{a}^{2}} \overline{\boldsymbol{u}} \cdot \nabla \frac{\partial p^{\prime}}{\partial t}+\frac{\bar{\gamma}}{\bar{a}^{2}} \frac{\partial p^{\prime}}{\partial t} \nabla \cdot \overline{\boldsymbol{u}} \\
& -\nabla \cdot\left(\bar{\rho} \boldsymbol{u}^{\prime} \cdot \nabla \boldsymbol{u}^{\prime}+\rho^{\prime} \frac{\partial \boldsymbol{u}^{\prime}}{\partial t}\right)+\frac{1}{\bar{a}^{2}} \frac{\partial}{\partial t}\left(\boldsymbol{u}^{\prime} \cdot \nabla p^{\prime}\right)+\frac{\bar{\gamma}}{\bar{a}^{2}} \frac{\partial}{\partial t}\left(p^{\prime} \nabla \cdot \boldsymbol{u}^{\prime}\right) \\
& +\nabla \cdot \mathscr{F}{ }^{\prime}-\frac{1}{\bar{a}^{2}} \frac{\partial \mathscr{P}^{\prime}}{\partial t}
\end{aligned}
$$

The boundary condition is set on the gradient of $p^{\prime}$, found by taking the scalar product of the outward normal vector with Eq. (37):

$$
\hat{n} \cdot \nabla p^{\prime}=-f
$$


and

$$
\begin{aligned}
f & =\bar{\rho} \frac{\partial \boldsymbol{u}^{\prime}}{\partial t} \cdot \hat{n}+\bar{\rho}\left(\bar{u} \cdot \nabla \boldsymbol{u}^{\prime}+\boldsymbol{u}^{\prime} \cdot \nabla \bar{u}\right) \cdot \hat{n}+\bar{\rho}\left(\boldsymbol{u}^{\prime} \cdot \nabla \boldsymbol{u}^{\prime}\right) \cdot \hat{n} \\
& +\rho^{\prime} \frac{\partial \boldsymbol{u}^{\prime}}{\partial t} \cdot \hat{n}-\mathscr{F}^{\prime} \cdot \hat{n}
\end{aligned}
$$

In the first instance, the intent of the approximate method is to replace the partial differential equations of conservation by an equivalent system of ordinary differential equations. This is accomplished by averaging over the volume of the chamber, using a version of the method of least residuals, essentially Galerkin's method. The procedure begins with multiplication of the equations by a suitable weighting function, followed by integration over the volume. According to the remarks in Sec. II, an appropriate choice of weighting function is the mode shape for the unperturbed motions. In the limiting case in which all perturbations are ignored, the unperturbed waves are governed by Eqs. (39) and (41), with $h=f=0$. There may be circumstances in which a different choice is more effective (e.g., the average Mach number is high and the influence of the exhaust nozzle on the wave motions is substantial), but here we base our analysis on the limiting case. Hence, the mode shape $\psi_{n}$ for the $n$th mode satisfies the following equations:

$$
\begin{array}{r}
\nabla^{2} \psi_{n}+k_{n}^{2} \psi_{n}=0 \\
\hat{n} \cdot \nabla \psi_{n}=0
\end{array}
$$

The procedure amounts to comparing the unperturbed problem with the actual problem to be analyzed. Multiply Eq. (39) by $\psi_{n}$ and (43a) by $p^{\prime}$, subtract the results, and integrate over the chamber:

$$
\begin{aligned}
& \int\left[\psi_{n} \nabla^{2} p^{\prime}-p^{\prime} \nabla^{2} \psi_{n}\right] \mathrm{d} V-\frac{1}{\bar{a}^{2}} \int \psi_{n} \frac{\partial^{2} p^{\prime}}{\partial t^{2}} \mathrm{~d} V \\
& \quad-k_{n}^{2} \int p^{\prime} \psi_{n} \mathrm{~d} V=\int \psi_{n} h \mathrm{~d} V
\end{aligned}
$$

Apply Green's theorem to the left-hand side, substitute the boundary conditions (41) and (43b), and rearrange the terms to give

$$
-\frac{1}{\bar{a}^{2}} \int \psi_{n} \frac{\partial^{2} p^{\prime}}{\partial t^{2}} \mathrm{~d} V-k_{n}^{2} \int \psi_{n} p^{\prime} \mathrm{d} V=\int \psi_{n} h \mathrm{~d} V+\oiint \psi_{n} f \mathrm{~d} S
$$

We now take explicit advantage of the observational result that the oscillations usually occurring in rocket motors are not very different from the classical acoustic modes. The unsteady pressure field is expressed as a synthesis of the normal modes $\psi_{m}(\boldsymbol{r})$, with time-varying amplitudes $\eta_{m}(t)$ :

$$
p^{\prime}(r, t)=\bar{p} \sum_{m=1}^{\infty} \eta_{m}(t) \psi_{m}(r)
$$


Correspondingly, the velocity field is written as

$$
\boldsymbol{u}^{\prime}(\boldsymbol{r}, t)=\sum_{m=1}^{\infty} \frac{\dot{\eta}_{m}(t)}{\bar{\gamma} k_{m}^{2}} \nabla \psi_{m}(\boldsymbol{r})
$$

These are clearly not exact representations of the true fields, for the boundary conditions are not satisfied; Eq. (45) gives $\hat{n} \cdot \nabla p^{\prime}=0$ because the $\psi_{m}$ satisfy Eq. (43b) and, according to Eq. (46), the velocity fluctuation vanishes on the boundary. This means that Eqs. (45) and (46) do not accurately reproduce the spatial structure of the unsteady motions near the boundary. Nevertheless, the errors are small when the perturbations contained in $h$ and $f$ are small, and because of the spatial averaging, the equations to be found for the amplitudes will provide a satisfactory basis for studying real problems. The boundary conditions influence the results through the function $f$ appearing in Eq. (44) and eventually as a contribution to $F_{n}$ [Eqs. (48) and (49)]. As we noted in Sec. I, the accuracy of the final results can be assessed quantitatively only by comparison with numerical solutions to the partial differential equations.

The set of normal modes can be constructed so that the $\psi_{n}(r)$ are orthogonal:

$$
\begin{aligned}
\int \psi_{m} \psi_{n} \mathrm{~d} V & =E_{n}^{2} \delta_{m n} \\
E_{n}^{2} & =\int \psi_{n}^{2} \mathrm{~d} V
\end{aligned}
$$

Substitute Eq. (45) in the left-hand side of Eqs. (44), and use the orthogonality property [Eqs. (47a) and (47b)] to find the desired system of equations for the amplitudes:

$$
\frac{\mathrm{d}^{2} \eta_{n}}{\mathrm{~d} t^{2}}+\omega_{n}^{2} \eta_{n}=F_{n}
$$

where $\omega_{n}=\bar{a} k_{n}$ and

$$
F_{n}=-\frac{\bar{a}^{2}}{\bar{p} E_{n}^{2}}\left\{\int h \psi_{n} \mathrm{~d} V+\oint f \psi_{n} \mathrm{~d} S\right\}
$$

The functions $h$ and $f$ contain $p^{\prime}$ and $u^{\prime}$, which are to be replaced by the approximate forms [Eqs. (45) and (46)]. Hence, the "force" $F_{n}$ depends on the amplitudes $\eta_{m}$. In general, both linear and nonlinear terms will appear, and the system of Eqs. (48) represents the time-dependent motions of a collection of nonlinear oscillators, one oscillator being associated with each classical mode.

For the one-dimensional approximation, the equations corresponding to $(39-42)$ are

$$
\begin{gathered}
\frac{1}{S_{c}} \frac{\partial}{\partial z}\left(S_{c} \frac{\partial p^{\prime}}{\partial z}\right)-\frac{1}{\bar{a}^{2}} \frac{\partial^{2} p^{\prime}}{\partial t^{2}}=h_{1} \\
\frac{\partial p^{\prime}}{\partial z}=-f_{1}(z=0, L)
\end{gathered}
$$


where $L$ is the length of the chamber, and

$$
\begin{aligned}
h_{1} & =-\bar{\rho} \frac{1}{S_{c}} \frac{\partial}{\partial z}\left(S_{c} \frac{\partial \bar{u} u^{\prime}}{\partial z}\right)+\frac{\bar{u}}{\bar{a}^{2}} \frac{\partial^{2} p^{\prime}}{\partial t \partial z}+\frac{\bar{\gamma}}{\bar{a}^{2}} \frac{\partial p^{\prime}}{\partial t} \frac{1}{S_{c}} \frac{\partial}{\partial z}\left(S_{c} \bar{u}\right) \\
& -\frac{1}{S_{c}} \frac{\partial}{\partial z} S_{c}\left(\bar{\rho} u^{\prime} \frac{\partial u^{\prime}}{\partial z}+\rho^{\prime} \frac{\partial u^{\prime}}{\partial t}\right) \\
& +\frac{1}{\bar{a}^{2}} \frac{\partial}{\partial z}\left(u^{\prime} \frac{\partial p^{\prime}}{\mathrm{d} z}\right)+\frac{\bar{\gamma}}{\bar{a}^{2}} \frac{\partial p^{\prime}}{\partial t} \frac{1}{S_{c}} \frac{\partial}{\partial z}\left(S_{c} u^{\prime}\right) \\
& +\frac{1}{S_{c}} \frac{\partial}{\partial z}\left(S_{c} \mathscr{F}_{1}^{\prime}\right)-\frac{1}{\bar{a}^{2}} \frac{\partial \mathscr{P}_{1}^{\prime}}{\partial t} \\
f_{1} & =\bar{\rho} \frac{\partial u^{\prime}}{\partial t}+\bar{\rho} \frac{\partial}{\partial z}\left(\bar{u} u^{\prime}\right)+\bar{\rho} u^{\prime} \frac{\partial u^{\prime}}{\partial z}+\rho^{\prime} \frac{\partial u^{\prime}}{\partial t}-\mathscr{F}_{1}^{\prime}
\end{aligned}
$$

The normal modes for one-dimensional motions are found as the solutions to

$$
\begin{gathered}
\frac{1}{S_{c}} \frac{\mathrm{d}}{\mathrm{d} z}\left(S_{c} \frac{\mathrm{d} \psi_{\ell}}{\mathrm{d} z}\right)+k_{\ell}^{2} \psi_{\ell}=0 \\
\frac{\mathrm{d} \psi_{\ell}}{\mathrm{d} z}=0(z=0, L)
\end{gathered}
$$

and the expansions for the acoustic field are

$$
\begin{aligned}
& p^{\prime}(z, t)=\bar{p} \sum_{j=1}^{\infty} \eta_{j}(t) \psi_{j}(z) \\
& u^{\prime}(z, t)=\sum_{j=1}^{\infty} \frac{\dot{\eta}_{j}(t)}{\bar{\gamma} k_{j}^{2}} \frac{\mathrm{d} \psi_{j}(z)}{\mathrm{d} z}
\end{aligned}
$$

Orthogonality of the normal modes is expressed as

$$
\begin{aligned}
\int_{0}^{L} \psi_{j} \psi_{\ell} S_{c} \mathrm{~d} z & =E_{\ell}^{2} \delta_{j l} \\
E_{\ell}^{2} & =\int_{0}^{L} \psi_{\ell}^{2} S_{c} \mathrm{~d} z
\end{aligned}
$$

With a procedure similar to that leading to Eq. (48), we find the equations for the amplitudes:

$$
\begin{gathered}
\frac{\mathrm{d}^{2} \eta_{\ell}}{\mathrm{d} t^{2}}+\omega_{\ell}^{2} \eta_{\ell}=F_{\ell} \\
F_{\ell}=-\frac{\bar{a}^{2}}{\bar{p} E_{\ell}^{2}}\left\{\int_{0}^{L} h_{1} \psi_{\ell} S_{c} \mathrm{~d} z+\left[f_{1} \psi_{\ell} S_{\epsilon}\right]_{0}^{L}\right\}
\end{gathered}
$$


We note that the classical modes used here are true standing wave motions having well-defined frequencies and phase differences among the spatial distributions that are invariant with time. Yet the general motions described by the set (48) and (56) are not so restricted: standing waves with energy losses or gains, traveling waves, and discrete wave motions (pulses) can all be analyzed with this formulation.

The progression from the partial differential equations to the ordinary differential equations [Eqs. (48)] is already a great simplification of the analysis. We could now apply Eqs. (48) directly, as Zinn and Powell ${ }^{9,10}$ did first in a similar treatment of combustion instabilities in liquid rockets. However, one further step applying the method of time averaging will replace the system of second-order equations by an equivalent system of first-order equations. This greatly reduces the cost of routine calculations and also provides a convenient basis for studying formally the general behavior of unsteady motions. The following argument applies to both the three-dimensional and one-dimensional formulations.

Time averaging is a reasonable procedure for practical problems, based on the observation that the oscillations commonly have amplitudes and phases varying slowly in time: their changes are small during one period of oscillation. Hence, the amplitudes $\eta_{n}(t)$ may be written in the form

$$
\eta_{n}(t)=r_{n}(t) \sin \left[\omega_{n} t+\phi_{n}(t)\right]=A_{n}(t) \sin \omega_{n} t+B_{n}(t) \cos \omega_{n} t
$$

The time-varying phase $\phi_{n}(t)$ is observed as a frequency shift, the actual frequency for the perturbed mode being $\mathrm{d} / \mathrm{d} t\left(\omega_{n} t+\phi_{n}\right)=\omega_{n}+\dot{\phi}_{n}$. Construction of the equations for $r_{n}(t)$ and $\phi_{n}(t)$ or, equivalently, $A_{n}(t)$ and $B_{n}(t)$ may be done at least two ways.

The first method ${ }^{11}$ is a modest variation of the method of averaging developed by Krylov and Bogoliubov. ${ }^{12}$ By analogy with a simple mass/ spring system, the energy $\mathscr{E}_{n}$ can be associated with the oscillator governed by Eq. (48):

$$
\mathscr{E}_{n}=1 / 2 \omega_{n}^{2} \eta_{n}^{2}+1 / 2 \dot{\eta}_{n}^{2}
$$

The instantaneous velocity of the oscillator is $\dot{\eta}_{n}$, so that the rate at which work is done on the oscillator is $\dot{\eta}_{n} F_{n}$. Averaged over an interval $\tau$ at time $t$, the values are

$$
\left\langle\mathscr{E}_{n}\right\rangle=\frac{1}{\tau} \int_{t}^{t+\tau} \mathscr{E}_{n}\left(t^{\prime}\right) \mathrm{d} t^{\prime}, \quad\left\langle\dot{\eta}_{n} F_{n}\right\rangle=\frac{1}{\tau} \int_{t}^{t+\tau} \dot{\eta}_{n} F_{n} \mathrm{~d} t^{\prime}
$$

Conservation of energy for the averaged motion implies that the rate of change of time-averaged energy should equal the time-averaged rate of power in

$$
\frac{\mathrm{d}}{\mathrm{d} t}\left\langle\mathscr{E}_{n}\right\rangle=\left\langle\dot{\eta}_{n} F_{n}\right\rangle
$$

Because the single function $\eta_{n}$ has been replaced by two functions, $r_{n}$ and $\phi_{n}$, we are free to place a restriction; following Krylov and Bogoliu- 
bov, ${ }^{12}$ we require

$$
\frac{\mathrm{d} \phi_{n}}{\mathrm{~d} t} r_{n} \cos \left(\omega_{n} t+\phi_{n}\right)+\frac{\mathrm{d} r_{n}}{\mathrm{~d} t} \sin \left(\omega_{n} t+\phi_{n}\right)=0
$$

Differentiating Eq. (58) and enforcing Eq. (62) gives the formula for the velocity in the same form as that for a classical conservative oscillator:

$$
\dot{\eta}_{n}=\omega_{n} r_{n} \cos \left(\omega_{n} t+\phi_{n}\right)
$$

and the energy is

$$
\mathscr{E}_{n}=1 / 2 \omega_{n}^{2} r_{n}^{2}
$$

With Eq. (63), Eq. (61) gives

$$
\frac{\mathrm{d} r_{n}}{\mathrm{~d} t}=\frac{1}{\omega_{n} \tau} \int_{t}^{t+\tau} F_{n} \cos \left(\omega_{n} t^{\prime}+\phi_{n}\right) \mathrm{d} t^{\prime}
$$

The equation for $\phi_{n}(t)$ is found by substituting Eqs. (58), (63), and (62) in the oscillator equation [Eq. (48)]; multiplication by $\sin \left(\omega_{n} t+\phi_{n}\right)$ and time averaging gives

$$
r_{n} \frac{\mathrm{d} \phi_{n}}{\mathrm{~d} t}=\frac{-1}{\omega_{n} \tau} \int_{t}^{t+\tau} F_{n} \sin \left(\omega_{n} t^{\prime}+\phi_{n}\right) \mathrm{d} t^{\prime}
$$

It is often more convenient to use the equations for $A_{n}(t)$ and $B_{n}(t)$, found by solving Eqs. (58), (62), (65), and (66) for $\dot{A}_{n}$ and $\dot{B}_{n}$, to give

$$
\begin{aligned}
& \frac{\mathrm{d} A_{n}}{\mathrm{~d} t}=\frac{1}{\omega_{n} \tau} \int_{t}^{t+\tau} F_{n} \cos \omega_{n} t^{\prime} \mathrm{d} t^{\prime} \\
& \frac{\mathrm{d} B_{n}}{\mathrm{~d} t}=\frac{-1}{\omega_{n} \tau} \int_{t}^{t+\tau} F_{n} \sin \omega_{n} t^{\prime} \mathrm{d} t^{\prime}
\end{aligned}
$$

The assumption that the amplitudes and phases are slowly varying means

$$
\begin{aligned}
& \dot{r}_{n} \tau \ll 1, \quad \dot{\phi}_{n} \tau \ll 2 \pi \\
& \dot{A}_{n} \tau \ll 1, \quad \dot{B}_{n} \tau \ll 1
\end{aligned}
$$

These inequalities imply that the functions $A_{n}(t)$ and $B_{n}(t)$ will be taken as constant under the integrals in Eqs. (67a) and (67b).

The original motivation for developing the method of averaging in the form expressed as Eqs. (67a) and (67b) was to provide the basis for treating arbitrarily shaped chambers. Differences between geometries are reflected in the unperturbed mode shapes and frequencies. The mode shapes affect 
the values of parameters that arise in Eqs. (67a) and (67b), but the frequency spectrum, as we shall see, influences the qualitative structure of the equations. It appears that the derivation of Eqs. (67a) and (67b) is not restricted to particular geometries, but, to date, these results have been applied only to the simplest case of longitudinal modes for which the harmonic frequencies are integral multiples of the fundamental. Application to other cases requires further calculations, which we will not pursue here; preliminary examination suggests some difficulties that have not been resolved.

A second method has recently been applied to problems of transverse modes in a circular cylinder. ${ }^{13,14}$ First we introduce a bookkeeping parameter $\varepsilon$ measuring the smallness of the right-hand side of Eq. (48):

$$
\frac{\mathrm{d}^{2} \eta_{n}}{\mathrm{~d} t^{2}}+\omega_{n}^{2} \eta_{n}=\varepsilon \bar{F}_{n}
$$

(In most problems, $\varepsilon$ is a Mach number characterizing the average flowfield; for the second-order acoustics treated here, the Mach numbers of the average and oscillating flows are of the same order.) Now $\tilde{F}_{n}$ is of the same order as $\eta_{n}$. As before, write $\eta_{n}(t)$ in the form (58), and impose the condition (62) written in terms of $A_{n}$ and $B_{n}$ :

$$
\frac{\mathrm{d} A_{n}}{\mathrm{~d} t} \sin \omega_{n} t+\frac{\mathrm{d} B_{n}}{\mathrm{~d} t} \cos \omega_{n} t=0
$$

Substitute Eq. (58) in Eq. (69); the result can be solved with Eq. (70) to give

$$
\begin{aligned}
\frac{\mathrm{d} A_{n}}{\mathrm{~d} t} & =\frac{\varepsilon}{\omega_{n}} \tilde{F}_{n} \cos \omega_{n} t \\
\frac{\mathrm{d} B_{n}}{\mathrm{~d} t} & =-\frac{\varepsilon}{\omega_{n}} \tilde{F}_{n} \sin \omega_{n} t
\end{aligned}
$$

These equations still involve no approximations beyond those required to produce Eq. (69). We now introduce two time scales, a "fast" scale, $1 / \omega_{n}$, proportional to the period of the oscillation; and a "slow" scale, $1 / \varepsilon \omega_{n}$, which characterizes the relatively gradual variations of the amplitude and phase. Correspondingly, the dimensionless fast and slow time variables are defined: $t_{f}=\omega_{n} t$ and $t_{s}=\varepsilon \omega_{n} t$.

In terms of these new variables, Eqs. (71a) and (71b) are

$$
\begin{aligned}
& \frac{\mathrm{d} A_{n}}{\mathrm{~d} t_{s}}=\frac{1}{\omega_{n}^{2}} \tilde{F}_{n} \cos t_{f} \\
& \frac{\mathrm{d} B_{n}}{\mathrm{~d} t_{s}}=-\frac{1}{\omega_{n}^{2}} \bar{F}_{n} \sin t_{f}
\end{aligned}
$$

The idea now is to average Eqs. (72a) and (72b) over an interval $\tau_{f}$ in the fast variable; because the $A_{n}$ and $B_{n}$ are functions of the slow variable, we 
have

$$
\begin{aligned}
& \frac{1}{\tau_{f}} \int_{t_{f}}^{t f+\tau_{f}} \frac{\mathrm{d} A_{n}}{\mathrm{~d} t_{s}} \mathrm{~d} t_{f}^{\prime} \approx \frac{\mathrm{d} A_{n}}{\mathrm{~d} t_{s}}=\frac{1}{\omega_{n}^{2} \tau_{f}} \int_{i f}^{t f+\tau_{f}} \tilde{F}_{n}\left(t_{s}, t_{f}^{\prime}\right) \cos t_{f}^{\prime} \mathrm{d} t_{f}^{\prime} \\
& \frac{1}{\tau_{f}} \int_{t_{f}}^{t f+\tau_{f}} \frac{\mathrm{d} B_{n}}{\mathrm{~d} t_{s}} \mathrm{~d} t_{f}^{\prime} \approx \frac{\mathrm{d} B_{n}}{\mathrm{~d} t_{s}}=-\frac{1}{\omega_{n}^{2} \tau_{f}} \int_{t_{s}}^{t+\tau_{f}} \tilde{F}_{n}\left(t_{s}, t_{f}^{\prime}\right) \sin t_{f}^{\prime} \mathrm{d} t_{f}^{\prime}
\end{aligned}
$$

As shown, $\vec{F}_{n}$ is a function of both the slow and fast time variables, because

$$
\eta_{i}=A_{i}\left(t_{s}\right) \sin \left(\frac{\omega_{i}}{\omega_{n}} t_{f}\right)+B_{i}\left(t_{s}\right) \cos \left(\frac{\omega_{i}}{\omega_{n}} t_{f}\right)
$$

When $\tilde{F}_{n}$ is formed, terms linear and quadratic in the $A_{i}, B_{i}$ are produced, weighted by sinusoids. Because they are functions of $t_{s}$, essentially constant during the interval $\tau_{f}$, all such factors can be taken outside the integrals in Eqs. (73a) and (73b). Hence, the averaging is performed on sinusoids having frequencies that are sums and differences of the natural frequencies. The terms that survive the averaging are then determined by the relative magnitudes of the periods compared with the period $2 \pi / \omega_{n}$ of the mode in question. Some terms have precisely zero frequency when the differenced frequencies vanish. Others oscillate at nonzero but low frequencies, with periods long compared with the averaging period. Some experience with special problems ${ }^{13,14}$ has shown that, if the frequency is less than roughly half the frequency of the mode in question, those terms should be retained. They contribute modulating factors on the right-hand sides of Eqs. (73a) and (73b), which are meaningful, as comparison with numerical solutions to the second-order Eqs. (48) have shown. Open questions concerning the use of time-averaging in general still remain.

Culick ${ }^{11}$ has shown that $F_{n}$ has the following form for second-order acoustics:

$$
F_{n}=-\sum_{i=1}^{\infty}\left[D_{n i} \dot{\eta}_{i}+E_{n i} \eta_{i}\right]-\sum_{i=1}^{\infty} \sum_{j=1}^{\infty}\left[A_{n i j} \dot{\eta}_{i} \dot{\eta}_{j}+B_{n i j} \eta_{i} \eta_{j}\right]
$$

The constants $D_{n i}, E_{n i}, A_{n i j}, B_{n i j}$ depend on the unperturbed mode shapes and frequencies; the $D_{n i}$ and $E_{n i}$ arise from linear processes and are usually proportional to the Mach number of the mean flow. (A notable exception arises with the presence of condensed material discussed in Sec. IV.D. The characteristic parameter then depends on the mass fraction of condensed material and on the properties of the particles.) Consider the linear terms only. Substitution of Eq. (75) in Eq. (73), or in Eqs. (67a) and (67b), with $\tau$ equal to the period of the $n$th mode, leads to the following results:

$$
\left(\frac{\mathrm{d} A_{n}}{\mathrm{~d} t}\right)_{\text {lincar }}=-\frac{1}{2} D_{n n} A_{n}-\frac{1}{2} \frac{E_{n n}}{\omega_{n}} B_{n}
$$




$$
\left(\frac{\mathrm{d} B_{n}}{\mathrm{~d} t}\right)_{\text {linear }}=-\frac{1}{2} D_{n n} B_{n}+\frac{1}{2} \frac{E_{n n}}{\omega_{n}} A_{n}
$$

Multiply the first of these by $A_{n}$ and the second by $B_{n}$, and add the results to find the equation for the amplitude, $\dot{r}_{n}=-\left(D_{n n} / 2\right) r_{n}$, where $r_{n}^{2}=$ $A_{n}^{2}+B_{n}^{2}$. Thus, $r_{n} \sim \exp \left(\alpha_{n} t\right)$, with $\alpha_{n}=-D_{n n} / 2$, the growth constant for the $n$th mode, as defined generally by Eq. (6).

Now, from the definition (58) of $\eta_{n}(t)$, we find

$$
A_{n} \approx e^{\alpha_{n} t} \cos \phi_{n}, \quad B_{n} \approx e^{\alpha_{n} t} \sin \phi_{n}
$$

Substitution in Eqs. (76a) and (76b) leads to the identification $E_{n n} / 2 \omega_{n}=$ $\dot{\phi}_{n}$. But $\dot{\phi}_{n}$ can be interpreted as the frequency shift in the $n$th mode that is due to the perturbations because the perturbed frequency is

$$
\omega=\frac{\mathrm{d}}{\mathrm{d} t}\left(\omega_{n} t+\phi_{n}\right)=\omega_{n}+\dot{\phi}_{n}=\omega_{n}+\left(\omega-\omega_{n}\right)
$$

Hence, we have established the two rules that the growth constant for the $n$th mode is $1 / 2$ the coefficient of $\dot{\eta}_{n}$ in the form (75), and the frequency shift is $1 / 2 \omega_{n}$ times the coefficient of $\eta_{n}$ :

$$
\begin{aligned}
\alpha_{n} & =-\frac{1}{2} D_{n n} \\
\theta_{n}=-\left(\omega-\omega_{n}\right) & =-\frac{1}{2} \frac{E_{n n}}{\omega_{n}}
\end{aligned}
$$

These rules are often conveniently applied after a representation of a particular process has been constructed. Examples are given in the following section.

\section{Prediction of Linear Stability}

Most contemporary predictions of linear stability are based on the analysis formulated in the preceding section. The results are readily programmed for routine computations; a widely used program is that prepared originally by Lovine et al. ${ }^{16}$ and recently in revised form by Nickerson et al. ${ }^{17}$

Whatever method is used, the starting point for three-dimensional problems is the pair of equations (37) and (38) with the nonlinear terms $\bar{\rho} \boldsymbol{u}^{\prime}$. $\nabla \boldsymbol{u}^{\prime}$, etc., dropped. In general, one would expect to need the linearized forms of Eq. (24) for conservation of mass, and of Eq. (22), the equation of state. However, for problems of small-amplitude motions, it is an adequate approximation to suppose that the fluctuations in the acoustic field are isentropic, so that $p \sim \rho^{\bar{\gamma}}$. Then the pressure and density changes are related by

$$
\frac{p^{\prime}}{\bar{p}}=\bar{\gamma} \frac{\rho^{\prime}}{\bar{\rho}}
$$


Temperature fluctuations may arise in the source terms, and so we shall need the linearized form of the equation of state [Eq. (22)]:

$$
\frac{p^{\prime}}{\bar{p}}=\frac{\rho^{\prime}}{\bar{\rho}}+\frac{T^{\prime}}{\bar{T}}
$$

Only the terms linear in $h$, Eq. (40), and $f$, Eq. (42), are retained. Hence, the forcing function $F_{n}$ defined in Eqs. (48) and (49) is linear in $p^{\prime}$ and $\boldsymbol{u}^{\prime}$. We may therefore introduce the exponential time dependence familiar in linear problems, $\eta_{n} \sim \exp (i \bar{a} k t)$,

$$
\eta_{n}=\hat{\eta}_{n} e^{\overline{\bar{a}} k t}, \quad h=\hat{h} e^{\overline{i a} k t}, \quad f=\hat{f} e^{\bar{a} k t}
$$

where $k$ is the complex wave number:

$$
k=\frac{1}{\bar{a}}(\omega-i \alpha)
$$

The real part of $\vec{a} k$ is the frequency of the $n$th mode shifted from the classical value $\omega_{n}$ by the perturbations proportional to the Mach number of the average flow. The imaginary part $\alpha$ is the growth constant for the perturbed $n$th mode: $\alpha>0$ if the mode is unstable. By construction, $\alpha$ is itself proportional to the average Mach number.

Thus, as far as linear behavior is concerned, the purpose of the approximate analysis is to provide a systematic procedure for computing the frequency shifts and growth constants associated with each of the classical acoustic modes. The idea is that an arbitrary small-amplitude unsteady motion can be represented as a Fourier synthesis of the classical unperturbed modes, with time-dependent coefficients $\eta_{n}(t)$, as expressed by Eqs. (45) and (46). If only one of the modes is unstable, then the general linear motion must be unstable. Hence, the linear stability of motions generally is established by determining the signs of all $\alpha$ for the classical normal modes: the same formula for $k$ applies to all modes, but the numerical values of $\omega$ and $\alpha$ depend on $n$.

Because Eq. (48) is linear, after substitution of the forms (80), the common factor $e^{i \bar{a} k t}$ cancels, and we find the formula for $k^{2}$ :

$$
k^{2} \equiv \frac{1}{\bar{a}^{2}}(\omega-i \alpha)^{2}=\frac{\omega_{n}^{2}}{\bar{a}^{2}}+\frac{1}{\bar{p} E_{n}^{2}}\left\{\int \frac{\hat{h}}{\hat{\eta}_{n}} \psi_{n} \mathrm{~d} V+\oiint \frac{\hat{f}}{\hat{\eta}_{n}} \psi_{n} \mathrm{~d} S\right\}
$$

Taking real and imaginary parts, and using the fact that, because they are both proportional to the average Mach number, $\alpha / \omega,\left(\omega-\omega_{n}\right) / \omega_{n}<<1$, we find the following formulas for the actual frequency and the growth constant:

$$
\begin{aligned}
& \omega=\omega_{n}+\frac{\bar{a}^{2}}{2 \omega_{n} \bar{p} E_{n}^{2}}\left\{\int \frac{\hat{h}^{(r)}}{\hat{\eta}_{n}} \psi_{n} \mathrm{~d} V+\oint \frac{\hat{f}^{(r)}}{\hat{\eta}_{n}} \psi_{n} \mathrm{~d} S\right\} \\
& \alpha=\frac{-\bar{a}^{2}}{2 \omega_{n} \bar{p} E_{n}^{2}}\left\{\int \frac{\hat{h}^{(i)}}{\hat{\eta}_{n}} \psi_{n} \mathrm{~d} V+\oint \frac{\hat{f}^{(i)}}{\hat{\eta}_{n}} \psi_{n} \mathrm{~d} S\right\}
\end{aligned}
$$


where superscripts $(r)$ and $(i)$ denote real and imaginary parts, respectively. Thus, the entire problem of linear stability has been reduced to evaluating the integrals over $\hat{h}$ and $\hat{f}$. This is a perfectly general result, restricted only by the assumption that the $\psi_{n}$ satisfies Eqs. (43a) and (43b) and by the modeling of the source terms in $h$ and $f$. Note that the meaning of smallness here implies that $\omega$ differs from $\omega_{n}$ and $\alpha$ differs from zero by terms of order $\vec{M}$.

\section{A. Evaluating the Integrals of $\boldsymbol{h}$ and $f$}

Some rearrangement leads to the following result for the linear parts of $h$ and $f$ :

$$
\begin{aligned}
& \int \hat{h} \psi_{n} \mathrm{~d} V+\oint \hat{f} \psi_{n} \mathrm{~d} S=\bar{\rho} k_{n}^{2} \int(\overline{\boldsymbol{u}} \cdot \hat{u}) \psi_{n} \mathrm{~d} V \\
& -\bar{\rho} \int(\hat{\boldsymbol{u}} \times \nabla \times \overline{\boldsymbol{u}}) \cdot \nabla \psi_{n} \mathrm{~d} V \\
& \quad+i \frac{k_{n}}{\bar{a}} \int \psi_{n}[\overline{\boldsymbol{u}} \cdot \nabla \hat{p}+\bar{\gamma} \hat{p} \nabla \cdot \overline{\boldsymbol{u}}] \mathrm{d} V \\
& \quad+\frac{i k_{n}}{\bar{a}} \int \psi_{n} \hat{P} \mathrm{~d} V-\int \hat{\mathscr{F}} \cdot \nabla \psi_{n} \mathrm{~d} V+i \bar{\rho} \bar{a} k_{n} \oiint_{\psi_{j}} \psi_{n} \hat{\boldsymbol{u}} \cdot \hat{n} \mathrm{~d} S
\end{aligned}
$$

The mean flowfield may be rotational $(\nabla \times \overline{\boldsymbol{u}} \neq 0)$, and sources are accommodated $(\nabla \cdot \overline{\boldsymbol{u}} \neq 0)$. Also, because the integrands are proportional to $\bar{M}$ and because $k$ differs from $k_{n}$ by terms of order $\bar{M}$, we have replaced $k$ by $k_{n}$, thereby correctly neglecting terms of order $\bar{M}^{2}$. It is important to understand that this analysis of linear stability gives consistent results to first order in the mean flow Mach number. To study linear stability, only the $n$th term is retained in the series expansions (45) and (46). Moreover, to the order to which the equations have been constructed, the acoustic quantities on the right-hand side of Eq. (84) must be replaced by their classical unperturbed values; thus, set $k=k_{n}$ and

$$
\hat{p}=\bar{p} \hat{\eta}_{n} \psi_{n}, \quad \hat{\boldsymbol{u}}=\frac{i \bar{a}}{\bar{\gamma} k_{n}} \hat{\eta}_{n} \nabla \psi_{n}
$$

After some calculations and rearrangement, we find

$$
\begin{aligned}
k^{2} & =k_{n}^{2}+\frac{\bar{a}^{2}}{\bar{p} E_{n}^{2}}\left\{i \bar{\rho} \bar{a} k_{n} \oiint\left(\frac{\hat{u}}{\hat{\eta}_{n}}+\frac{1}{\bar{\gamma}} \bar{u} \psi_{n}\right) \cdot \hat{n} \psi_{n} \mathrm{~d} S\right. \\
& +i(\bar{\gamma}-1) \frac{k_{n}}{\bar{a}} \bar{p} \int(\nabla \cdot \bar{u}) \psi_{n}^{2} \mathrm{~d} V \cdots i \frac{k_{n}}{\bar{a}} \int \frac{\hat{\rho}}{\hat{\eta}_{n}} \psi_{n} \mathrm{~d} V \\
& \left.-\int \frac{\hat{\mathscr{P}}}{\hat{\eta}_{n}} \cdot \nabla \psi_{n} \mathrm{~d} V\right\}
\end{aligned}
$$

Taking the real and imaginary parts of this result and substituting in Eqs. (83a) and (83b), gives the basic formulas for treating the problem of linear 
stability:

$$
\begin{aligned}
\omega=\omega_{n} & +\frac{\bar{a}^{2}}{2 \omega_{n} \bar{p} E_{n}^{2}}\left\{\frac{k_{n}}{\bar{a}} \int \psi_{n} \frac{\hat{\vartheta}^{(i)}}{\hat{\eta}_{n}} \mathrm{~d} V-\int \frac{1}{\hat{\eta}_{n}} \hat{\mathscr{F}}(r) \cdot \nabla \psi_{n} \mathrm{~d} V\right. \\
\left.-\bar{\rho} \bar{a} k_{n} \oint \frac{1}{\hat{\eta}_{n}}(\hat{\boldsymbol{u}} \cdot \hat{n})^{(i)} \psi_{n} \mathrm{~d} S\right\} & \\
\alpha & =-\frac{\bar{a}^{2}}{2 \omega_{n} \bar{p} E_{n}^{2}}\left\{(\bar{\gamma}-1) \frac{k_{n}}{\bar{a}} \bar{p} \int \psi_{n}^{2}(\nabla \cdot \overline{\boldsymbol{u}}) \mathrm{d} V\right. \\
& -\frac{k_{n}}{\bar{a}} \int \psi_{n} \frac{\hat{\rho}^{(r)}}{\hat{\eta}_{n}} \mathrm{~d} V-\int \frac{1}{\hat{\eta}_{n}} \hat{\xi}(t) \cdot \nabla \psi_{n} \mathrm{~d} V \\
& \left.+\bar{\rho} \bar{a} k_{n} \oint\left[\frac{1}{\hat{\eta}_{n}}(\hat{\boldsymbol{u}} \cdot \hat{n})^{(r)} \psi_{n}+\frac{1}{\bar{\gamma}} \psi_{n}^{2} \bar{u} \cdot \hat{n}\right] \mathrm{d} S\right\}
\end{aligned}
$$

We now examine the most important contributions to linear stability.

\section{B. Admittance and Response Functions}

The term $\gamma \hat{u}$ in the surface integral arises from the contribution $\bar{\rho} \partial u / \partial t$ in $f$. Here $\hat{u}$ is not replaced by its unperturbed value $(\hat{u} \cdot \hat{n}=0)$ at the surface because, in general, the boundary is not rigid. At burning surfaces, for example, the unsteady combustion process produces fluctuations of burning rate, and hence velocity, of the order of the average Mach number.

It is a convention in classical acoustics that has become standard practice in this subject, to replace fluctuations of the velocity at the boundary by admittance functions. The idea is that, if a small pressure fluctuation is imposed on a boundary, the surface will move, at a velocity proportional, in first approximation, to the pressure fluctuation. In solid rockets, there are chiefly three classes of boundaries: inert impermeable surfaces; burning surfaces; and areas through which flow may pass, mainly the exhaust nozzle.

No exposed surface in a solid rocket chamber is truly inert, but erosion of insulation material is slow compared with combustion rates. Thus, we may consider the material to be inert as a good first approximation. In that case, there is negligible motion of the surface, and the acoustic field is influenced primarily by viscous effects confined to an acoustic boundary layer, treated in the following section.

Burning surfaces and regions of flow through the boundary may be treated together. From the definition of mass flux, $m_{b}=-\rho_{s} u \cdot \hat{n}$, and with the perfect-gas law, we have

$$
-\hat{\boldsymbol{u}} \cdot \hat{n}=\frac{\hat{m}_{b}}{\bar{\rho}_{s}}-\overline{\boldsymbol{u}} \cdot \hat{n} \frac{\Delta \hat{T}_{s}}{\bar{T}_{s}}+\frac{\hat{p}}{\bar{\gamma} \bar{p}} \overline{\boldsymbol{u}} \cdot \hat{n}
$$

The minus sign appears on $\boldsymbol{u} \cdot \hat{n}$ because $\hat{n}$ is positive outward but $\boldsymbol{u}$ and $m_{b}$ are positive inward. The quantity $\Delta \hat{T}_{s}$ represents the difference between the actual temperature change and the isentropic temperature fluctuation 
associated with the pressure disturbance:

$$
\Delta \hat{T}_{s}=\hat{T}_{s}-\frac{\bar{\gamma}-1}{\bar{\gamma}} \frac{\hat{p}}{\bar{p}} \bar{T}_{s}
$$

With $\bar{\gamma} \bar{p}=\bar{\rho} \bar{a}^{2}$, and $\hat{p} / \bar{p}=\hat{\eta}_{n} \psi_{n}$, Eq. (89) can be solved for the combination appearing in the first integral of Eq. (86):

$$
\frac{\bar{\gamma}}{\hat{\eta}_{n}}(\hat{\boldsymbol{u}} \cdot \hat{n}) \psi_{n}+(\bar{u} \cdot \hat{n}) \psi_{n}^{2} \equiv-\bar{\gamma}\left(\frac{\hat{m}_{b}}{\bar{\rho}_{b}}-\overline{\boldsymbol{u}} \cdot \hat{n} \frac{\Delta \hat{T}_{s}}{\bar{T}_{s}}\right) \frac{\psi_{n}}{\hat{\eta}_{n}}
$$

Analysis of the unsteady response of a burning surface produces most directly results for fluctuations of the mass flux $\hat{m}_{b}$, whereas measurements provide directly the combination on the left-hand side of Eq. (91). Hence, two functions have been introduced in the literature, the response function $R_{b}$ and the admittance function $A_{b}$, defined by the relations

$$
\begin{aligned}
\frac{\hat{m}_{b}}{\bar{m}_{b}} & =R_{b} \frac{\hat{p}}{\bar{\gamma} \bar{p}} \\
-\frac{\hat{\boldsymbol{u}} \cdot \hat{n}}{\bar{a}} & =A_{b} \frac{\hat{p}}{\bar{\gamma} \bar{p}}
\end{aligned}
$$

With these definitions, the combination (91) gives

$$
\frac{1}{\bar{a}}\left(\bar{\gamma} \frac{\hat{u} \cdot \hat{n}}{\hat{\eta}_{n} \psi_{n}}+\bar{u} \cdot \hat{n}\right) \equiv-\left(A_{b}+\bar{M}_{b}\right) \equiv-\bar{M}_{b}\left(R_{b}+\gamma \frac{\Delta \hat{T}_{s} / \bar{T}_{s}}{\hat{\eta}_{n} \psi_{n}}\right)
$$

where the subscript $b$ has been introduced to indicate conditions at the burning surface.

Because the processes at the burning surface are the source of the energy for instabilities, the problem of coupling to acoustical motions has received much attention. It is not possible to compute accurate values of the response function for a given propellant. Experimental methods carry considerable uncertainties but have advanced to the point of effectiveness for comparing propellants and for assessing trends of behavior accompanying compositional changes.

Denison and Baum ${ }^{18}$ first discovered an approximation to the response function now commonly used for correlating data and in computations of stability. Culick ${ }^{19}$ reviewed the available analyses of the response function. Because of common assumptions of the physical behavior, almost all results have the same form as Denison and Baum's:

$$
R_{b}=\frac{n A B \bar{\gamma}}{\lambda+\frac{A}{\lambda}-(1+A)+A B}
$$

The complex function of frequency $\lambda$ is computed as the solution to the equation

$$
\lambda(\lambda-1)=i \Omega
$$


where $\Omega=\kappa_{p} \omega / \bar{r}_{b}^{2}$ is proportional to the ratio of the period of the thermal wave in the solid to the period of the imposed acoustic oscillation. With $n$ specified as the index in the burning rate law, the formula (94) gives values of $R_{b}$ depending on the two parameters $A$ and $B$, which themselves are functions of the properties of the propellant. Each analysis of the unsteady behavior produces explicit formulas for $A$ and $B$, so that their values depend partly on the approximations used. However, with the broad assumptions commonly used (notably that the burning is one dimensional and the gas phase responds quasistatically), Eq. (94) is found. Figure 1 shows the real and imaginary parts for typical values of the parameters.

Similarly, the influence of the nozzle is represented by an admittance function, but with a sign change to account for the fact that the flow is outward from the chamber. Also, it is assumed that the flow is isentropic, and so corresponding to Eq. (93), we have

$$
\frac{\overline{\boldsymbol{y}}}{\overline{\bar{a}}}\left(\frac{\hat{\boldsymbol{u}} \cdot \hat{n}}{\hat{\boldsymbol{\eta}}_{n} \psi_{n}}\right)+\frac{\overline{\boldsymbol{u}} \cdot \hat{n}}{\bar{a}} \equiv\left(A_{N}+\bar{M}_{N}\right)
$$

Substitution of the preceding results in Eqs. (87) and (88) gives

$$
\begin{aligned}
& \omega=\omega_{n}+\frac{\bar{a}^{2}}{2 \omega_{n} \bar{p} E_{n}^{2}}\left\{\frac{k_{n}}{\bar{a}} \int \psi_{n} \frac{\hat{\hat{P}}^{(i)}}{\hat{\eta}_{n}} \mathrm{~d} V-\int \frac{1}{\hat{\eta}_{n}} \hat{\mathscr{F}}(r) \cdot \nabla \psi_{n} \mathrm{~d} V\right.
\end{aligned}
$$

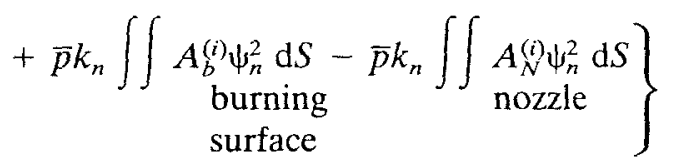

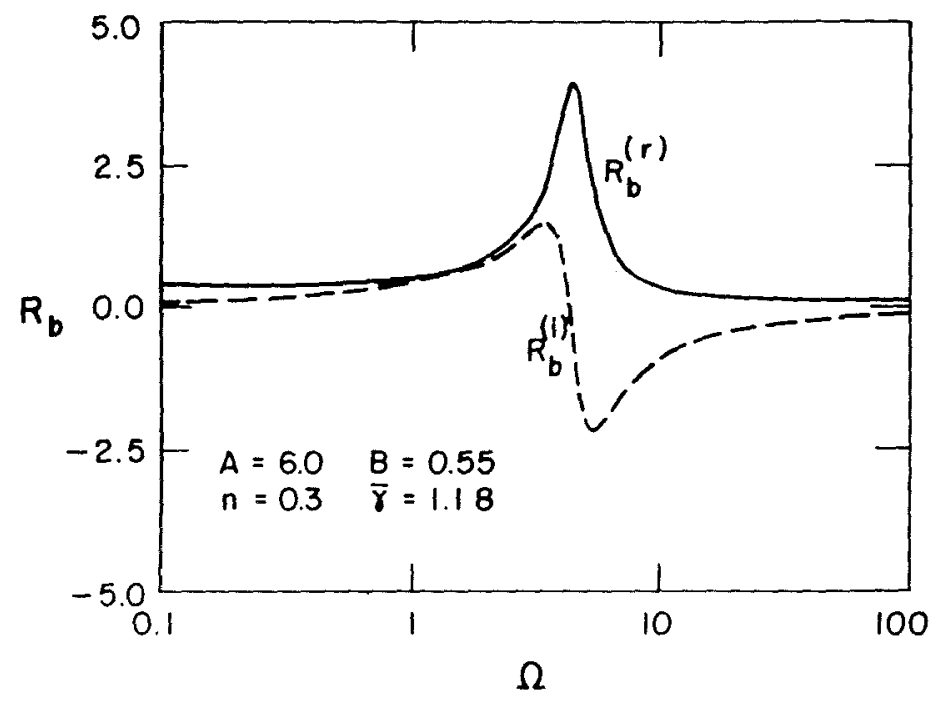

Fig. 1 Frequency spectrum of combustion response function. 


$$
\begin{aligned}
& \alpha=-\frac{\bar{a}^{2}}{2 \omega_{n} \bar{p} E_{n}^{2}}\left\{(\bar{\gamma}-1) \frac{k_{n}}{\bar{a}} \bar{p} \int \psi_{n}^{2}(\nabla \cdot \bar{u}) \mathrm{d} V\right. \\
& -\frac{k_{n}}{\bar{a}} \int \psi_{n} \frac{\hat{\mathscr{P}}^{(r)}}{\hat{\eta}_{n}} \mathrm{~d} V-\int \frac{1}{\hat{\eta}_{n}} \hat{\mathscr{H}}^{(i)} \cdot \nabla \psi_{n} \mathrm{~d} V
\end{aligned}
$$

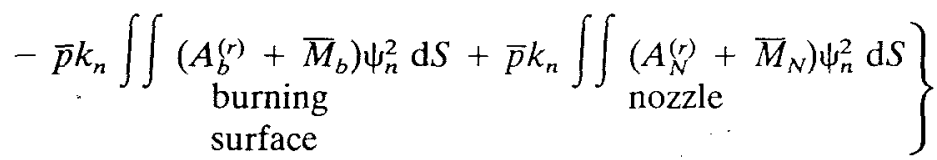

Normally, $A_{b}^{(r)}$ and $A_{N}^{(r)}$, as defined here, are both positive: the burning surface tends to drive waves, and the nozzle tends to attenuate. Their contributions to the growth constant, extracted from Eq. (98), are

$$
\begin{gathered}
\alpha_{C}=\frac{\bar{a}}{2 E_{n}^{2}} \iint_{\text {burning }}\left(A_{b}^{(r)}+\bar{M}_{b}\right) \psi_{n}^{2} \mathrm{~d} S \\
\text { surface } \\
\alpha_{N}=-\frac{\bar{a}}{2 E_{n}^{2}} \iint_{\text {nozzle }}\left(A_{N}^{(r)}+\bar{M}_{N}\right) \psi_{n}^{2} \mathrm{~d} S
\end{gathered}
$$

Thus, in accord with the foregoing remarks, $\alpha_{C}>0$ and $\alpha_{N}<0$ in the usual case.

Suppose that the nozzle entrance is located at $z=L$ and that $A_{N}^{(r)}+$ $\bar{M}_{N}$ is constant over its area $S_{N}$; then Eq. (100) simplifies to

$$
\alpha_{N}=-\frac{\bar{a}}{2 L}\left(A_{N}^{(r)}+\bar{M}_{N}\right)\left[\frac{L \iint_{z=L} \psi_{n}^{2} \mathrm{~d} S}{\int \psi_{n}^{2} \mathrm{~d} V}\right]
$$

The ratio in square brackets is constant, or nearly so. This result shows that, for a given nozzle and entrance Mach number (i.e., ratio of burning surface area to entrance area), the attenuation constant due to the nozzle is inversely proportional to the length of the motor.

Similarly, if $A_{b}^{(r)}+\bar{M}_{b}$ is constant over the burning surface, Eq. (99) is

$$
\alpha_{C}=\frac{\bar{a}}{2}\left(A_{b}^{(r)}+\bar{M}_{b}\right)\left[\frac{\iint \psi_{n}^{2} \mathrm{~d} S}{\int \psi_{n}^{2} \mathrm{~d} V}\right]
$$

in which the surface integral is over the burning surface. The ratio in brackets depends significantly on the location of the burning surface. If the geometry is fixed, so is the function $\psi_{n}^{2}$. Consequently, $\alpha_{C}$ is larger if the burning surface is placed in the vicinity of antinodes of the $n$th mode. 
This effect may cause the value of $\alpha_{C}$ to be significantly different for the various modes in a given chamber.

\section{Attenuation at Inert Surfaces}

Losses of acoustic energy at an inert surface are due to the actions of viscous stresses and heat conduction. Those processes occur in a relatively thin layer near the wall but may be accommodated, in the general framework constructed here, by suitable interpretation of the force $\mathscr{F}^{\prime}$ in the momentum equation (37) and of the heat source $Q^{\prime}$ in Eq. (38) for the pressure. The force is derived from a stress tensor $\overleftrightarrow{\tau}$, and the heat source is associated with the heat flux vector $q$; the fluctuations are

$$
\begin{aligned}
& \mathscr{F}_{v}^{\prime}=\nabla \cdot \overleftrightarrow{\tau}^{\prime} \\
& Q_{v}^{\prime}=\nabla \cdot q^{\prime}
\end{aligned}
$$

where subscript $v$ denotes viscous effects.

The contributions from these terms to the formula (88) for the growth constant are

$$
\begin{aligned}
\alpha_{V} & =\frac{\bar{a}^{2}}{2 \omega_{n} \bar{p} E_{n}^{2}}\left\{\frac{k_{n}}{\bar{a}} \frac{\bar{R}}{\bar{C}_{V}} \int \psi_{n} \frac{1}{\hat{\eta}_{n}}(\nabla \cdot \hat{q})^{(r)} \mathrm{d} V\right. \\
& \left.+\int \frac{1}{\hat{\eta}_{n}}(\nabla \cdot \hat{\leftrightarrow})^{(i)} \cdot \nabla \psi_{n} \mathrm{~d} V\right\}
\end{aligned}
$$

Both $q^{\prime}$ and $\overleftrightarrow{\tau}^{\prime}$ are significant only in the thin acoustic boundary layer adjacent to the wall. If the layer thickness is sufficiently small compared to the radius of curvature of the wall, we may treat the viscous processes as in parallel flow past a flat plate. Let $y$ denote the coordinate normal to the wall, measured positive inward, so that the element of volume near the surface is $\mathrm{d} V=\mathrm{d} y \mathrm{~d} S$. Within this approximation, only the $y$ component of $\boldsymbol{q}^{\prime}$ is nonzero, and so

$$
\hat{Q}=\frac{\partial \hat{q}_{y}}{\partial y}
$$

The viscous stress is parallel to the surface and varies only in the $y$ direction:

$$
\hat{F}=\frac{\partial \hat{\tau}_{y}}{\partial y} \hat{k}
$$

where $\hat{k}$ is the unit vector in the flow direction, say, $z$.

Within the acoustic boundary layer, $\psi_{n}$ and $\nabla \psi_{n}$ are approximated by their values at the surface and depend only on $z$. Hence, the volume integrals in Eq. (104) may be written as

$$
\int \psi_{n} \nabla \cdot \hat{q} \mathrm{~d} V \approx \iint \mathrm{d} S \psi_{n} \int_{0}^{\infty} \frac{\partial \hat{q}_{y}}{\partial y} \mathrm{~d} y=-\iint \hat{q}_{w} \psi_{n} \mathrm{~d} S
$$




$$
\int \hat{F}^{\prime} \cdot \nabla \psi_{n} \mathrm{~d} V \approx \iint \mathrm{d} S \nabla \psi_{n} \cdot \int_{0}^{\infty} \frac{\partial \hat{\tau}_{y}}{\partial y} \hat{k} \mathrm{~d} y=-\iint \hat{\tau}_{w} \frac{\partial \psi_{n}}{\partial z} \mathrm{~d} S
$$

Far from the surface, both $\hat{q}_{y}^{\prime}$ and $\hat{\tau}_{y}^{\prime}$ vanish so that the integrals may be extended to $y \rightarrow \infty$ as shown. The viscous stress at the wall is $\hat{\tau}_{w}=-(\mu \partial \hat{u})$ $\partial y)_{w}$, where $u^{\prime}$ is the velocity fluctuation in the direction of flow and the heat flux is $\hat{q}_{w}=-(k \partial \hat{T} / \partial y)_{w}$. Hence, the formula (104) becomes

$$
\begin{aligned}
\alpha_{v} & =-\frac{\bar{a}^{2}}{2 \omega_{n} \bar{p} E_{n}^{2}}\left\{\frac{k_{n}}{\bar{a}} \frac{\bar{R}}{\bar{C}_{V}} \iint \frac{1}{\hat{\eta}_{n}}\left(k \frac{\partial \hat{T}}{\partial y}\right)_{w}^{(r)} \psi_{n} \mathrm{~d} S\right. \\
& \left.+\iint \frac{1}{\hat{\eta}_{n}}\left(\mu \frac{\partial \hat{u}}{\partial y}\right)_{w}^{(i)} \frac{\partial \psi_{n}}{\mathrm{~d} z} \mathrm{~d} S\right\}
\end{aligned}
$$

The solutions for the velocity and temperature fluctuations in the acoustic boundary layer are

$$
\begin{aligned}
& u^{\prime}=\hat{u}_{x}\left[1-e^{-\lambda y}\right] e^{i \omega t} \\
& T^{\prime}=\hat{T}_{x}\left[1-e^{-\sqrt{P r \lambda y}}\right] e^{i \omega t}
\end{aligned}
$$

where $\hat{u}_{x}, \hat{T}_{x}$ are the amplitudes far from the wall and

$$
\begin{aligned}
& \lambda=\frac{1}{\delta}(1+i) \\
& \delta=\sqrt{2 v / \omega}
\end{aligned}
$$

Thus, $\delta$ is roughly the thickness of the acoustic boundary layer, and the factor $(1+i)$ in $\lambda$ represents the existence of shear waves within the layer. From Eqs. (109a) and (109b), we have

$$
\begin{aligned}
& \left(\mu \frac{\partial u^{\prime}}{\partial y}\right)_{w}=\frac{\mu}{\delta}(1+i) \hat{u}_{x} e^{i \omega t} \\
& \left(k \frac{\partial T^{\prime}}{\partial y}\right)_{w}=\frac{k}{\delta} \sqrt{\operatorname{Pr}}(1+i) \hat{T}_{x} e^{i \omega t}
\end{aligned}
$$

Before these results are used in Eq. (108), the phases must be corrected. One way to do this is first to convert from the complex to real forms containing the time dependence $\eta_{n}$ or $\dot{\eta}_{n}$. According to the definitions introduced by Eq. (46), the acoustic velocity outside the boundary layer is $\dot{\eta}_{n} \nabla \psi_{n} / \bar{\gamma} k_{n}^{2}$. Here we use only the component parallel to the surface; hence, $\nabla \psi_{n} \rightarrow \partial \psi_{n} / \partial z$. Thus, in Eq. (111a), we must replace $\hat{u}_{x} e^{i \omega t}$ by $\dot{\eta}_{n}\left(\partial \psi_{n} / \partial z\right) / \bar{\gamma} k_{n}^{2}$. The factor $i$ introduces a time derivative, $i \omega e^{i \omega t}=\partial\left(e^{i \omega t}\right) /$ $\partial t$, so that $i \hat{u}_{x} e^{i \omega t}$ is replaced by $\ddot{\eta}_{n}\left(\partial \psi_{n} / \partial z\right) / \bar{\gamma} \omega_{n} k_{n}^{2}$. But to the order required 
here, $\ddot{\eta}_{n} \approx-\omega_{n}^{2} \eta_{n}$, and Eq. (111a) becomes

$$
\left(\mu \frac{\partial u^{\prime}}{\partial y}\right)_{w}=\frac{\mu}{\delta} \frac{1}{\bar{\gamma} k_{n}^{2}}\left(\dot{\eta}_{n}-\omega_{n} \eta_{n}\right) \frac{\partial \psi_{n}}{\partial z}
$$

Outside the boundary layer, the temperature is related to the pressure by the formula for isentropic variations and

$$
\frac{T_{x}^{\prime}}{\bar{T}}=\frac{\bar{\gamma}-1}{\bar{\gamma}} \frac{p^{\prime}}{\bar{p}}
$$

with $p^{\prime}=\bar{p} \eta_{n} \psi_{n}$. Accordingly, Eq. (111b) is written as

$$
\left(k \frac{\partial T^{\prime}}{\partial y}\right)_{w}=\frac{k}{\delta} \sqrt{\operatorname{Pr}}\left(\frac{\bar{\gamma}-1}{\bar{\gamma}}\right) \bar{T}\left(\eta_{n}+\frac{1}{\omega} \dot{\eta}_{n}\right) \psi_{n}
$$

Now, set $\eta_{n}=\dot{\eta}_{n} e^{i \omega_{n} t}$ in Eqs. (112) and (113), take the real or imaginary parts as required, and substitute in Eq. (108) to find

$$
\alpha_{V}=-\frac{\sqrt{\omega_{n} \bar{v} / 2}}{2 E_{n}^{2}} \iint_{\substack{\text { inert } \\ \text { surface }}}\left[\left(\frac{\nabla_{\|} \psi_{n}}{k_{n}}\right)^{2}+\frac{\bar{\gamma}-1}{\sqrt{P r}} \psi_{n}^{2}\right] \mathrm{d} S
$$

For generality, $\partial \psi_{n} / \partial z$ has been replaced by the gradient $\nabla_{\|}$of $\psi_{n}$ parallel to the wall. For a longitudinal mode in a straight cylindrical tube, $\psi_{n}=$ $\cos k_{n} z$, and Eq. (114) gives the well-known result for the decay constant for a standing wave:

$$
\alpha_{V}=-\frac{2}{D} \sqrt{\frac{\omega_{n} \bar{v}}{2}}\left(1+\frac{\bar{\gamma}-1}{\sqrt{P r}}\right)
$$

where $D$ is the diameter of the tube.

Figure 2 shows one way of presenting the content of Eq. (115). The decay constant for a standing wave in a tube is made dimensionless by referring its value to the frequency. Note that the dependence on Prandtl number is very weak; the value $\bar{\gamma}=1.2$ is typical for combustion products in a solid rocket.

The dimensionless combination $\omega / \alpha$ is $2 \pi$ times the number of cycles required for the amplitude of oscillation to be reduced to $1 / e$ of its initial value. That interpretation is readily established from the definition of $\alpha$, the $p^{\prime} \sim e^{-\alpha t}$. The number of cycles executed in the time interval $\Delta t$ is $\Delta t /$ $\tau$ and $\tau=2 \pi / \omega$. Hence, one can write

$$
\alpha \Delta t=\alpha \tau \frac{\Delta t}{\tau}=2 \pi \frac{\alpha}{\omega} \frac{\Delta t}{\tau}
$$

The amplitude is reduced by the factor $1 / e$ if $\alpha \Delta t=1$; the last equation 


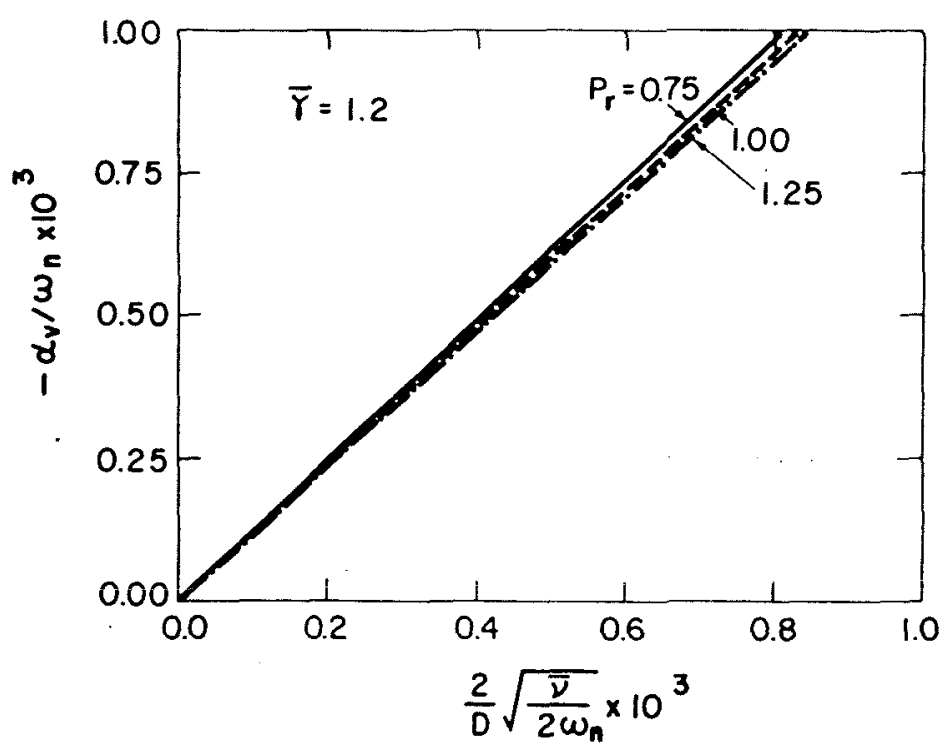

Fig. 2 Attenuation of acoustic waves by viscous damping.

then gives

$$
\frac{\omega}{\alpha}=2 \pi \frac{\Delta t}{\tau}=2 \pi \times \text { (number of cycles) }
$$

\section{Attenuation Due to Condensed Material}

Particularly if the propellant contains metal, some of the combustion products appear as condensed liquid or solid particles. Under suitable circumstances, the viscous interactions between the particles and the gas may provide a significant dissipation of acoustic energy. For rockets using metallized propellants, this is often the largest contribution to stability of small-amplitude oscillations. The amount of damping depends mainly on three quantities: the mass fraction $C_{m}$ of condensed material, the size of the particles, and the frequency of the oscillations. Perhaps the most significant practical consequence of the analysis summarized here is the result that, for a given frequency, there is a particle size (diameter) for which the attenuation per particle is maximum. That conclusion has been confirmed in practice and is the basis for one important means of treating instabilities in motors.

Attentuation of sound by suspended particles in a gas was first treated theoretically more than 75 years ago. The modern theory began with the work of Epstein and Carhart. ${ }^{20} \mathrm{~A}$ simplified analysis and experimental confirmation of the results have been provided by Temkin and Dobbins. ${ }^{21,22}$ The calculations discussed here constitute an alternative method fitting naturally in the approximate analysis. Extensive work by Kraeutle et al. ${ }^{23}$ has shown that this approach works in practice. 
The linear gas/particle interactions arise from Eqs. (18a) and (18b) written to first order in the fluctuations:

$$
\begin{aligned}
\delta \boldsymbol{F}_{p}^{\prime} & =-\overline{\boldsymbol{\rho}}_{p} \frac{\partial \delta \boldsymbol{u}_{p}^{\prime}}{\partial t} \\
\delta Q_{p}^{\prime} & =-\overline{\boldsymbol{\rho}}_{p} C \frac{\partial \delta T_{p}^{\prime}}{\partial t}
\end{aligned}
$$

The parts of $F^{\prime}$ and $P^{\prime}$ due to these terms only are found from the linearized forms of Eqs. (28) and (29):

$$
\begin{aligned}
& \mathscr{F}_{p}^{\prime}=\delta \mathscr{F}_{p}^{\prime}=-\bar{\rho}_{p} \frac{\partial \delta \boldsymbol{u}_{p}^{\prime}}{\partial t} \\
& \mathscr{P}^{\prime}=\frac{\bar{R}}{\overline{\bar{C}}_{V}} \delta Q_{p}^{\prime}=-\bar{\rho}_{p} \bar{R} \frac{C}{\bar{C}_{V}} \frac{\partial \delta T_{p}^{\prime}}{\partial t}
\end{aligned}
$$

Thus, Eqs. (97) and (98) give the contributions to the frequency shift and the growth constant:

$$
\begin{gathered}
\left(\omega-\omega_{n}\right)_{P} \equiv \delta \omega_{P}=\frac{-\bar{a}^{2}}{2 \omega_{n} \bar{p} E_{n}^{2}}\left\{\frac{k_{n}}{\bar{a}} \frac{\bar{R}}{\bar{C}_{V}} C \bar{\rho}_{p} \int \frac{1}{\eta_{n}}\left(\frac{\partial \delta T_{p}^{\prime}}{\partial t}\right)^{(i)} \psi_{n} \mathrm{~d} V\right. \\
\left.-\bar{\rho}_{p} \int \frac{1}{\eta_{n}}\left(\frac{\partial \delta u_{p}^{\prime}}{\partial t}\right)^{(r)} \cdot \nabla \psi_{n} \mathrm{~d} V\right\} \\
\alpha_{P}=\frac{-\bar{a}^{2}}{2 \omega_{n} \bar{p} E_{n}^{2}}\left\{\frac{k_{n}}{\bar{a}} \frac{\bar{R}}{\bar{C}_{V}} C \bar{\rho}_{p} \int \frac{1}{\eta_{n}}\left(\frac{\partial \delta T_{p}^{\prime}}{\partial t}\right)^{(r)} \psi_{n} \mathrm{~d} V\right. \\
\left.+\bar{\rho}_{p} \int \frac{1}{\eta_{n}}\left(\frac{\partial \delta \boldsymbol{u}_{p}^{\prime}}{\partial t}\right)^{(i)} \cdot \nabla \psi_{n} \mathrm{~d} V\right\}
\end{gathered}
$$

We have assumed, a good approximation in most practical cases, that the mass of particles per unit volume, $\rho_{p}$, and the mass fraction are nearly independent of position in the chamber.

To find $\delta u_{p}^{\prime}$ and $\delta T_{p}^{\prime}$, we treat the motions as locally one dimensional and solve the problem of single particle motion, $\boldsymbol{u}_{p}^{\prime}(t)$ and $T_{p}^{\prime}(t)$ being the velocity and temperature, respectively, of a particle located in a gas having oscillatory velocity $\boldsymbol{u}^{\prime}(t)$ and $T^{\prime}(t)$. Temperature gradients within a particle are ignored. See, for example, Rangal and Sirignano ${ }^{5}$ for a discussion of problems in which this assumption is not made. Moreover, we also ignore the effects of vaporization and combustion of the particles. In the absence of combustion, condensation or vaporization causes increased attenuation of acoustic waves. ${ }^{24,25} \mathrm{We}$ assume tentatively that the motions are such that the Reynolds number based on the relative speed, $\left|\boldsymbol{u}_{p}^{\prime}-\boldsymbol{u}^{\prime}\right|$, is less than unity. The approximation of Stokes' flow then applies, and the equa- 
tions of motion are

$$
\begin{aligned}
\frac{\mathrm{d} \boldsymbol{u}_{p}^{\prime}}{\mathrm{d} t} & =-\frac{18 \mu}{\rho_{s} \sigma^{2}}\left(\boldsymbol{u}_{p}^{\prime}-\boldsymbol{u}^{\prime}\right) \\
\frac{\mathrm{d} T_{p}^{\prime}}{\mathrm{d} t} & =-\frac{12 k}{\rho_{s} C \sigma^{2}}\left(T_{p}^{\prime}-T^{\prime}\right)
\end{aligned}
$$

where $\boldsymbol{u}_{p}^{\prime}$ is the velocity in the same direction as $\boldsymbol{u}^{\prime}$ and $\sigma$ is the particle diameter. These equations can be rewritten as

$$
\begin{aligned}
& \frac{\mathrm{d} \delta \boldsymbol{u}_{p}^{\prime}}{\mathrm{d} t}+\frac{1}{\tau_{d}} \delta \boldsymbol{u}_{p}^{\prime}=-\frac{\mathrm{d} \boldsymbol{u}^{\prime}}{\mathrm{d} t} \\
& \frac{\mathrm{d} \delta T_{p}^{\prime}}{\mathrm{d} t}+\frac{1}{\tau_{t}} \delta T_{p}^{\prime}=-\frac{\mathrm{d} T^{\prime}}{\mathrm{d} t}
\end{aligned}
$$

The relaxation times are

$$
\begin{aligned}
\tau_{d} & =\frac{\rho_{s} \sigma^{2}}{18 \mu} \\
\tau_{t} & =\left(\frac{3}{2} \frac{C \mu}{k}\right) \tau_{d}
\end{aligned}
$$

With $\boldsymbol{u}^{\prime}=\left(\dot{\eta}_{n} / \bar{\gamma} k_{n}^{2}\right) \nabla \psi_{n}$ and $T^{\prime} / \bar{T}=(\bar{\gamma}-1) \eta_{n} \psi_{n} / \bar{\gamma}$, the steady-state solutions $(t \rightarrow \infty)$ to Eqs. (121a) and (121b) are

$$
\begin{aligned}
& \delta u_{p}^{\prime}=X_{1}\left(\eta_{n}-\tau_{d} \dot{\eta}_{n}\right) \frac{1}{\bar{\gamma} k_{n}^{2}} \nabla \psi_{n} \\
& \delta T_{p}^{\prime}=-X_{2}\left(\tau_{t} \eta_{n}+\frac{\dot{\eta}_{n}}{\omega_{n}^{2}}\right) \frac{\bar{\gamma}-1}{\bar{\gamma}} \bar{T} \psi_{n}
\end{aligned}
$$

Dependence on frequency and particle properties is contained chiefly in the two functions $X_{1}$ and $X_{2}$ :

$$
\begin{aligned}
& X_{1}=\frac{\omega_{n} \Omega_{d}}{1+\Omega_{d}^{2}} \\
& X_{2}=\frac{\omega_{n} \Omega_{t}}{1+\Omega_{t}^{2}}
\end{aligned}
$$

where $\Omega_{d}=\omega_{n} \tau_{d}$ and $\Omega_{t}=\omega_{n} \tau_{t}$. For use in Eqs. (119) and (120), the time derivatives of Eqs. (123a) and (123b) are required. This produces terms containing $\dot{\eta}_{n}$ and $\ddot{\eta}_{n}$. To be consistent, we replace $\ddot{\eta}_{n}$ by $-\omega_{n}^{2} \eta_{n}$ and, after setting $\eta_{n}=\hat{\eta}_{n} e^{i \omega_{n} t}$, we eventually find the results

$$
\alpha_{P}=-\frac{1}{2} \frac{C_{m}}{1+C_{m}}\left[X_{1} \frac{1}{E_{n}^{2}} \oint\left(\frac{\nabla \psi_{n}}{k_{n}}\right)^{2} \mathrm{~d} V+(\bar{\gamma}-1) \frac{C}{\bar{C}_{p}} X_{2}\right]
$$




$$
\delta \omega_{P}=\frac{1}{2} \frac{C_{m}}{1+C_{m}}\left[\Omega_{d} X_{1} \frac{1}{E_{n}^{2}} \oiint\left(\frac{\nabla \psi_{n}}{k_{n}}\right)^{2} \mathrm{~d} V+(\bar{\gamma}-1) \frac{C}{\bar{C}_{p}} \Omega_{t} X_{2}\right]
$$

Equations (125a) and (125b), normalized to the angular frequency $\omega_{n}$, are plotted in Figs. 3 and 4 for longitudinal oscillations. The independent variable is $\omega_{n} \tau_{d}, 2 \pi$ times the ratio of the relaxation time for relative motion [see Eq. (122a)] to the period of the motion. According to Eq. (122a), the dominant influence on the relaxation time is the particle diameter $\tau_{d} \sim \sigma^{2}$. For typical solid propellants and operating conditions, the diameters of particles may range from fractions of a micron to tens of microns. The results shown in the figures have been computed for longitudinal oscillations in a chamber of constant cross section, and so $\psi_{n}=\cos k_{n} z=\cos (\pi n z)$ $L)$. In this case, Eqs. (125a) and (125b) reduce to

$$
\begin{gathered}
\alpha_{F}=-\frac{1}{2} \frac{C_{m}}{1+C_{m}}\left[X_{1}+(\bar{\gamma}-1) \frac{C}{\bar{C}_{p}} X_{2}\right] \\
\delta \omega_{P}=\frac{1}{2} \frac{C_{m}}{1+C_{m}}\left[\Omega_{d} X_{1}+(\bar{\gamma}-1) \frac{C}{\bar{C}_{p}} \Omega_{t} X_{2}\right]
\end{gathered}
$$

The most striking feature of the curves in Fig. 3 is that, for a fixed value of mass loading $C_{m}$, the dimensionless attenuation constant has a maximum value. That is, according to the interpretation expressed by Eq. (116), the number of cycles of oscillation required to reduce the amplitude by $1 / e$ is minimum. Thus, for a fixed frequency, there is a best value of relaxation time, that is, particle size, for obtaining maximum attenuation. This result has served as a successful practical guide to treating combustion instabilities

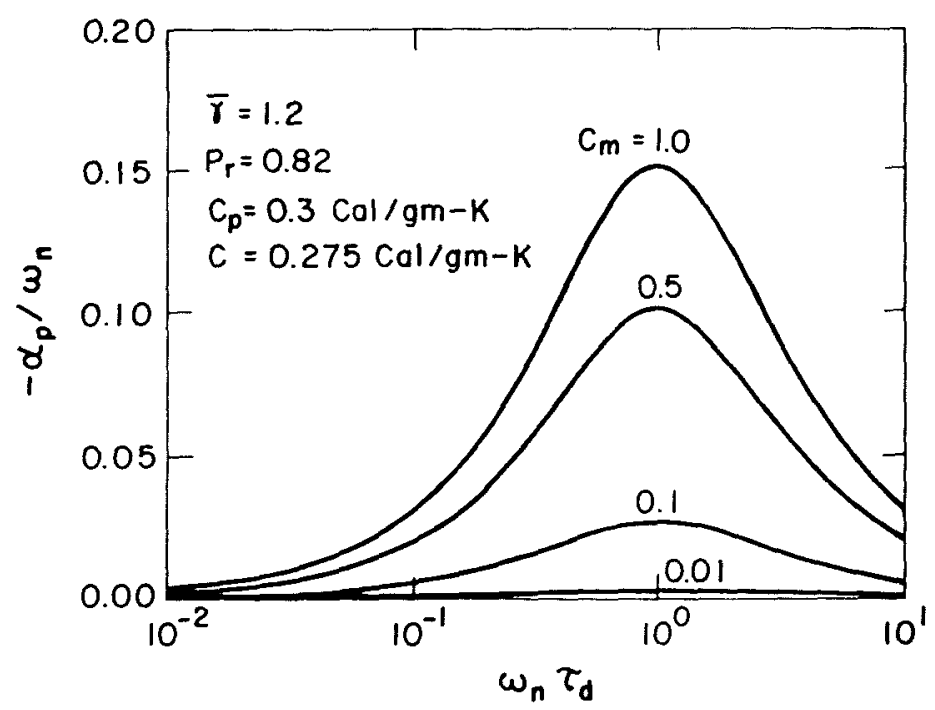

Fig. 3 Attenuation of acoustic waves by small particles suspended in a gas. 


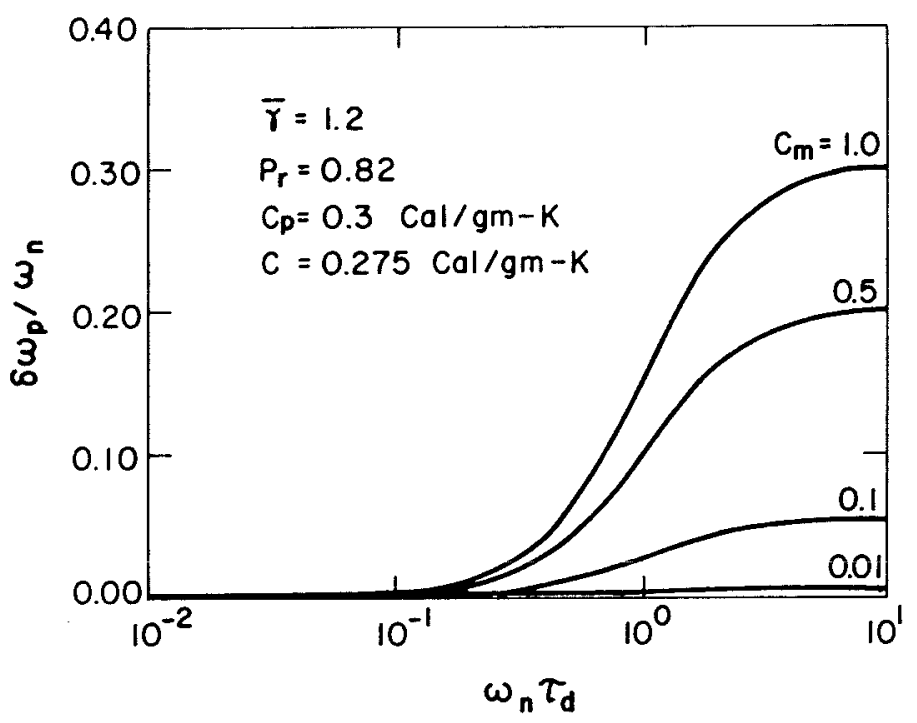

Fig. 4 Frequency shift of acoustic oscillation by small particles suspended in a gas.

in motors. Addition of inert particles having appropriate sizes, or altering the propellants in other ways, has reduced the amplitudes of oscillations.

Figure 4 shows a strong dependence of the frequency shift on both particle mass loading and on $\omega_{n} \tau_{d}$ or, as reasoned earlier, on particle size. The behavior is better understood by recognizing that, in a fixed geometry (here a tube, length $L$ ), the wavelength is fixed so that, from the fundamental relation $a=f \lambda=\omega \lambda / 2 \pi$, a frequency shift is equivalent to a change in the speed of sound:

$$
\frac{\delta \omega}{\omega}=\frac{\delta a}{a}
$$

Note especially in Fig. 3 that, as $\omega_{n} \tau_{d}$ approaches unity, when the attenuation constant is maximum, the change in the speed of sound is not a small perturbation if the particle mass loading is greater than 0.5 . The mass loading as a function of aluminum content $\mu$ in the solid propellant is given by the formula

$$
C_{m}=\frac{1.89 \mu}{1-1.89 \mu}
$$

If the propellant contains $15 \%$ aluminum $(\mu=0.15), C_{m}=0.4$, substantial shifts in the speed of sound occur. That is why this effect of particles was included in the formulation of the conservation equations [Eqs. (2426)]. The speed of sound given by Eq. (23), has the value shown in Fig. 4 for $\omega_{n} \tau_{d} \rightarrow \infty$.

The dependence of the frequency shift on $\omega_{n} \tau_{d}$ may be interpreted as follows. According to Eq. (122a), the relaxation time is proportional to the square of the particle diameter, and so $\omega_{n} \tau_{d} \sim \omega_{n} \sigma^{2}$. For low frequencies 
or small particles, $\omega_{n} \tau_{d} \rightarrow 0$; according to Fig. 4 , the frequency shift and change in the speed of sound, Eq. (127), vanish. In either case--slow unsteady motions with finite particle sizes or vanishingly small particles exposed to unsteady motions - the viscous losses in the flow about the particles become negligible. Hence, there can be no frequency shift, a result to be expected by analogy with the behavior of the resonant frequency of a classical mass/spring/dashpot system.

On the other hand, if the frequency is relatively high, even with small particles, or the particles are large, the viscous stresses cause substantial motions of the particles. When $\omega_{n} \tau_{d}$ is sufficiently small, the particles follow the gas motion very closely. The gas/particle mixture then behaves as a single fluid having density equal to the sum of the mass of gas and condensed material per unit volume, Eq. (19), but the compressibility is provided by the gas. Hence, the speed of sound assumes the equilibrium value given as Eq. (23), with mass-averaged thermodynamic properties.

The preceding results rest crucially on the assumptions of Stokes' flow and rapid decay of transient motions so that Eqs. (123a) and (123b) apply. It is an easy calculation to show that the Reynolds number based on the relative velocity exceeds unity for realistic particle sizes $(1-10 \mu)$ and quite modest amplitudes of oscillation. Hence, it appears that a nonlinear analysis of gas/particle interactions is required to cover conditions arising in practice. However, in all current applications, including the SSP program, ${ }^{17}$ the linear results are used. Kraeutle et al. ${ }^{23}$ based their experimental confirmations on the assumption of linear behavior, and it is likely that nonlinear effects cannot be detected within the experimental uncertainties.

The only calculations of the attenuation constant including nonlinear effects ${ }^{26}$ show that the linear results tend to be conservative. For a fixed frequency, increasing the amplitude of oscillation broadens the curves in Fig. 3 and moves the peak to slightly larger particle size; the maximum value of the attenuation is practically constant with amplitude. More recently, Levine et al. ${ }^{27}$ have produced some interesting results for the damping of nonlinear waves but with linear gas/particle interactions. The problem of nonlinear attenuation probably merits careful analysis, but the prospects are not especially promising for experimental verification.

\section{E. Mean Flow/Acoustics Interactions}

In classical acoustics, the presence of a nonuniform flowfield has usually been examined in connection with refraction of sound waves. For a stationary observer, the flow speed appears as a change in the speed of sound, which may be interpreted as a shift in the index of refraction. That effect must be present in a combustion chamber, but when the acoustical motions are approximately stationary waves, it has not been helpful to try to isolate that influence. In addition to refraction, there are several phenomena of mean flow/acoustics interactions that, after spatial averaging, combine to provide the term $\psi_{n}^{2} \bar{u} \cdot \hat{n}$ in the last integral of Eqs. (86) and (88).

The term in question appears to be simply the convection of acoustic energy by the mean flow through the boundary surface. It leads eventually to the contributions $\bar{M}_{b}$ in Eq. (99) for the burning surface and $\bar{M}_{N}$ in Eq. 
(100) for the exhaust nozzle. However, it is important to realize that the term $\psi_{n}^{2} \overline{\boldsymbol{u}} \cdot \hat{n}$ arises from several parts of Eq. (84) and includes contributions from interactions of both the acoustic velocity and pressure with the average flow. They may be traced back to terms like $\bar{u} \cdot \nabla u^{\prime}+\boldsymbol{u}^{\prime} \cdot \nabla \bar{u}, \bar{u} \cdot \nabla p^{\prime}$, etc., in the linearized conservation equations. That is, there are losses and gains of acoustic energy associated with processes within the volume as well as at the boundary. The final result is simple but has a complicated ancestry.

On the other hand, as Cantrell and Hart ${ }^{28}$ have shown, the same result is obtained if one formulates the global balance of acoustic energy for the chamber: the rate of change of acoustic energy within the volume equals the net flow of enthalpy through the boundary. The advantages of this approach is that the formula for the growth constant is obtained more directly; and it is quite easy to obtain the result valid to higher Mach numbers of the mean flow. The disadvantages are that the result for the frequency shift is not found; and, more important, other volumetric processes involving exchange of energy between the acoustic and mean flowfields are not easily included. Moreover, that formulation does not provide a basis for analyzing the dynamical nonlinear behavior of instabilities.

The mean flow/acoustics discussed so far are those associated with the existence of standing (or slowly varying) waves in a nonuniform but continuous mean flowfield. If the chamber contains obstructions or rapid changes of the boundary shape, the mean flow may separate, causing shear layers that are usually unstable and produce vortex shedding. Interactions between the vortices and the acoustic field are another source (or sink) of acoustic energy. Direct coupling between vortices and the acoustic field was first suggested by Flandro and Jacobs ${ }^{29}$ as a possible cause of instabilities. However, the coupling is weak - the vortices appear as quadrupole sources-and this is probably not an important mechanism in practice. Culick and Magiawala ${ }^{30}$ used simple apparatus to show that, if the vortices strike a barrier downstream, resonances are easily excited in a duct. This mechanism was investigated further by Nomoto and Culick, ${ }^{31}$ Flandro and Finlaysen ${ }^{32}$ and Aaron and Culick, ${ }^{33}$ and was confirmed by Dunlap and Brown $^{34}$ for flow in a scale model of an operational rocket. Extensive results have been reported by Schadow et al. ${ }^{35}$ for flow in geometries appropriate to ramjet combustors. The most recent and thorough analysis of this phenomenon has been given by Flandro. ${ }^{36}$

It appears that interactions between vortices and the acoustic field will not produce instabilities having large amplitudes. The likely reason is that the source of energy (the mean flow) is relatively small compared with that associated with combustion processes, and the coupling between the vortices and the acoustic field is weak. However, the point has not been proved-not even upper bounds on the possible amplitudes have been established. Vortex/acoustics interactions can cause instabilities, which may be troublesome even at low amplitudes if they fall in an undesirable frequency range. We shall not discuss them further because the subject still contains unresolved issues not suitable for discussion in this brief survey.

There is another contribution from mean flow/acoustics that does not arise in the three-dimensional analysis given earlier and that is often a significant contribution to stability. It is associated with interactions near 
the lateral boundary when the acoustic motions are parallel to the surface and the mean flow is perpendicular. First discussed by Culick, ${ }^{8}$ it has since acquired the name "flow-turning." This contribution to the loss of acoustic energy follows as a natural consequence of the one-dimensional analysis. It may be regarded, approximately, as the unsteady counterpart of the pressure loss accompanying flow in a duct with mass addition at the boundary (see, for example, Ref. 37, p. 234).

The formula for the complex wave number with the one-dimensional approximation is found by assuming the time dependence $e^{i \bar{a} k t}$ and using Eqs. (56) and (57). Corresponding to Eq. (82) for three-dimensional motions, we find

$$
k^{2}=k_{\ell}^{2}+\frac{1}{\bar{p} E_{\ell}^{2}}\left\{\int_{0}^{L} \frac{\hat{h}_{1}}{\hat{\eta}_{\ell}} \psi_{\ell} S_{c} \mathrm{~d} z+\left[\frac{\hat{f}_{1}}{\hat{\eta}_{\ell}} \psi_{\ell} S_{c}\right]_{0}^{L}\right\}
$$

Although there are additional contributions associated with the flow of particles inward at the lateral boundary, we shall treat only a pure gas flow to simplify the results. Then $\mathscr{F}_{1}=\mathscr{F}_{1 s}$ in Eq. (34) and $\mathscr{P}_{1}=\mathscr{P}_{1 s}$ in Eq. (35). The linear fluctuations of $\mathscr{F}_{1 s}$ and $P_{1 s}$ are

$$
\begin{aligned}
& \mathscr{F}_{1}^{\prime}=\mathscr{F}_{1 s}^{\prime}=\frac{1}{S_{c}} \int\left(u_{s}^{\prime}-u^{\prime}\right) \bar{m}_{b} \mathrm{~d} q+{ }^{\prime} \\
& \mathscr{P}_{1}^{\prime}=\mathscr{P}_{1 s}^{\prime}=\frac{R}{C_{v}} \frac{1}{S_{c}} \int\left[\gamma R T^{\prime} \bar{m}_{b}+\gamma R \bar{T}_{b}^{\prime}+\gamma R \Delta T_{s}^{\prime} \bar{m}_{b}\right] \mathrm{d} q
\end{aligned}
$$

where $\Delta T_{s}=T_{s}-T$ is the difference between the temperature of the flow injected at the boundary and that of the bulk flow. The value of $\Delta T_{s}^{\prime}$ depends theoretically on the model chosen for unsteady combustion of the solid (see, for example, Culick ${ }^{19}$ and Krier et al. ${ }^{38}$ ). Its value for actual systems is not known. The temperature fluctuation in the chamber is approximated by the value for isentropic motions, $T^{\prime}=(\gamma-1)(\bar{T} / \gamma \bar{p}) p^{\prime}$, so that the definition (90) is recovered.

Without the nonlinear terms in the definitions (52a) and (52b), the formula (128) becomes

$$
\left.\begin{array}{r}
k^{2}=k_{\ell}^{2}+\frac{1}{\bar{p} E_{\ell}^{2}}\left\{i \bar{\rho} \bar{a} k_{\ell}\left[\left(\frac{\hat{u}}{\hat{\eta}_{\ell}}+\frac{1}{\gamma} \bar{u} \psi_{\ell}\right) \psi_{\ell} S_{c}\right]_{0}^{L}\right. \\
-i \bar{\rho} \bar{a} k_{\ell} \int_{0}^{L} \frac{1}{S_{c}}\left[\frac{1}{\hat{\eta}_{\ell}}\left(\frac{\hat{m}_{b}}{\bar{\rho}}+\frac{\bar{m}_{b}}{\bar{\rho}} \frac{\Delta \hat{T}_{s}}{\bar{T}}\right) \mathrm{d} q \psi_{\ell} S_{c} \mathrm{~d} z\right. \\
+i \frac{k_{\ell} \bar{p}}{\bar{a}} \int_{0}^{L} \frac{1}{k_{\ell}^{2}}\left(\frac{\mathrm{d} \psi_{\ell}}{\mathrm{d} z}\right)^{2} \frac{1}{S_{c}} \int \frac{\bar{m}_{b}}{\bar{\rho}} \mathrm{d} q S_{c} \mathrm{~d} z
\end{array}\right]
$$

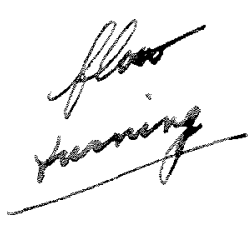


The last term in brackets represents the effect of injecting flow having axial speed at the boundary; for a burning solid propellent, this should be close to zero and will not be included here: we set $\hat{u}_{s}=\bar{u}_{s}=0$.

The first term in brackets, in Eq. (131), corresponds exactly to the first term of Eq. (86). It represents the influences of fluctuating flow normal to the boundary, and the net effect of several mean flow/acoustics interactions, including convection through the boundary. The second term can be put in the same form with the following calculations. From the definition of mass flux, we have $\hat{m}_{b}=\bar{\rho}_{s} \hat{u}_{b}+\hat{\rho}_{s} \bar{u}_{b}$, and with the perfect-gas law, $\hat{\rho}_{s} / \bar{\rho}_{s}=\hat{p} / \bar{p}-\hat{T}_{s} / \bar{T}_{s}$. Then, replace $\hat{T}_{s}$ by the definition (90) of $\Delta \hat{T}_{s}$, and rearrange the result to find the identity

$$
\frac{1}{\hat{\eta}_{\ell}}\left(\frac{\hat{m}_{b}}{\bar{\rho}_{s}}+\bar{u}_{b} \frac{\Delta \hat{T}_{s}}{\bar{T}_{s}}\right) \equiv \frac{\hat{u}_{b}}{\eta_{\ell}}+\frac{1}{\gamma} \bar{u}_{b} \psi_{\ell}
$$

We assume $\bar{\rho}_{s}=\bar{\rho}$ and, after the preceding observations have been accommodated, Eq. (131) becomes

$$
\begin{aligned}
k^{2} & =k_{\ell}^{2}+\frac{1}{\bar{p} E_{\ell}^{2}}\left\{i \bar{\rho} \bar{a} k_{\ell}\left[\left(\frac{\hat{u}}{\hat{\eta}_{\ell}} \psi_{\ell}+\frac{1}{\gamma} \bar{u} \psi_{\ell}^{2}\right) S_{c}\right]_{0}^{L}\right. \\
& -i \bar{\rho} \bar{a} \mathrm{k}_{\ell} \int_{0}^{L} \frac{1}{S_{c}} \int\left(\frac{\hat{u}_{b}}{\hat{\eta}_{\ell}}+\frac{1}{\gamma} \bar{u}_{b} \psi_{\ell}\right) \mathrm{d} q \psi_{\ell} S_{c} \mathrm{~d} z \\
& \left.+i \frac{k_{\ell} \bar{p}}{\bar{a}} \int_{0}^{L} \frac{1}{k_{\ell}^{2}}\left(\frac{\mathrm{d} \psi_{\ell}}{\mathrm{d} z}\right)^{2} \frac{1}{S_{c}} \int \bar{u}_{b} \mathrm{~d} q S_{c} \mathrm{~d} z\right\}
\end{aligned}
$$

This result should be compared with Eq. (86) for the three-dimensional case. The first two terms here may be deduced directly from the surface integral in Eq. (86). Careful analysis shows that there is one-to-one correspondence between all terms in the two cases, including those representing the influences of particulate material, except that the last term in Eq. (133) is new. That is what has come to be called the "flow-turning" effect.

The term "flow-turning" has been used because the term in question arises from the inelastic acceleration of injected fluid from its initial direction perpendicular to the surface, to its final state parallel to the surface. This process necessarily involves viscous stresses in the actual flow and therefore produces losses of energy (see Culick ${ }^{39}$ for further comments). The formulation within the one-dimensional approximation is indeed merely an approximation to the correct representation. No more precise analysis of the phenomenon exists although some relevant numerical analysis is in progress. ${ }^{40}$ Attempts to measure the associated losses have produced only qualitative confirmation. ${ }^{41}$

It is particularly important to emphasize two points: the acoustical energy loss associated with the flow-turning effect is often a substantial contribution to the stability of longitudinal motions in a motor, and the flow- 
turning losses are distinct from those associated with the acoustic boundary layer discussed in Sec. IV.C. The second item has often been misunderstood. Flandro ${ }^{61}$ carried out an analysis that essentially accounts for the influences of injected flow on the acoustic boundary layer. Because the flow far from the surface is in the perpendicular direction, in that calculation, there is no possibility of capturing the flow-turning effect.

The real and imaginary parts of Eq. (133) give the shift of frequency and attenuation constant corresponding to Eqs. (87) and (88):

$$
\begin{aligned}
& \omega=\omega_{n}+\frac{\bar{a}^{2}}{2 \omega_{\ell} \bar{p} E_{\ell}^{2}}\left\{-\bar{\rho} \bar{a} k_{\ell}\left[\left(\frac{\hat{u}^{(i)}}{\hat{\eta}_{\ell}} \psi\right) S_{c}\right]_{0}^{L}\right. \\
& \left.+\bar{\rho} \bar{a} k_{\ell} \int_{0}^{L} \frac{1}{S_{c}} \int\left(\frac{\hat{u}_{b}^{(i)}}{\hat{\eta}_{\ell}}\right) \mathrm{d} q \psi_{\ell} S_{c} \mathrm{~d} z\right\} \\
& \alpha=-\frac{\bar{a}^{2}}{2 \omega_{\ell} \bar{p} E_{\ell}^{2}}\left\{\frac{r}{\rho} \bar{a} k_{\ell}\left[\left(\frac{\hat{u}^{(r)}}{\hat{\eta}_{\ell}} \psi_{\ell}+\frac{1}{\gamma} \bar{u} \psi_{\ell}^{2}\right) S_{c}\right]_{0}^{L}\right. \\
& -\bar{\rho} \bar{a} k_{\ell} \int_{0}^{L} \frac{1}{S_{c}} \int\left(\frac{\hat{u}_{b}^{(r)}}{\hat{\eta}_{\ell}}+\frac{1}{\gamma} \bar{u}_{b} \psi_{\ell}\right) \mathrm{d} q \psi_{\ell} S_{c} \mathrm{~d} z \\
& \left.+\frac{k_{\ell} \bar{p}}{\bar{a}} \int_{0}^{L} \frac{1}{k_{\ell}^{2}}\left(\frac{\mathrm{d} \psi_{\ell}}{\mathrm{d} z}\right)^{2} \frac{1}{S_{c}} \int \bar{u}_{b} \mathrm{~d} q S_{c} \mathrm{~d} z\right\}
\end{aligned}
$$

These formulas show that the flow-turning effect, represented by the last term in Eq. (135), does not contribute to the frequency shift. Like the surface terms proportional to $\bar{u} \psi_{\hat{\ell}}^{2}$, it affects only the attenuation constant. Culick $^{3}$ has argued that the energy losses due to flow-turning must arise whenever the acoustical velocity is parallel to the burning surface. Consequently, the term should be interpreted as an energy loss per unit area of surface and should be included in the analysis of all combustion instabilities. The reason it does not appear in the usual three-dimensional theory, developed earlier, is that viscous effects have been ignored. As we noted earlier, the flow-turning loss arises within the one-dimensional analysis as an approximation to essentially viscous effects. The point is not universally accepted: see, for example, Van Moorhem, ${ }^{42}$ who seems to have misinterpreted some of the essential aspects of the analytical framework described here.

\section{F. Influence of Geometry on the Classical Mode Shapes, One-Dimensional Motions}

Although unsteady physical processes contribute to frequency shift and distortion of the mode structures, the first-order influences are generally due to the geometry of a combustion chamber. For practical applications, 
computation of the classical mode shapes is a necessary and often expensive part of a stability analysis. It is possible to obtain accurate results using finite difference or finite element methods. The expense is associated chiefly with constructing a suitable grid. To illustrate the matter here, we restrict ourselves to examples of one-dimensional motions. Laboratory tests have shown that the approximation works well for practical configurations. ${ }^{43,44}$

We consider the case illustrated in Fig. 5, a chamber in which there is a single abrupt change of cross-sectional area. The geometry is characterized by two parameters: the area ratio $S_{1} / S_{2}$, and the location $L_{1} / L$, at which the area changes. Often, the exhaust nozzle may cause a significant frequency shift, an effect that can be interpreted in first order as a shift $\Delta L$ of the velocity node at the exit of the chamber. We account for this contribution without extra labor by considering the length of the chamber to be slightly increased. The total length $L$ is measured from the rigid head end to a position displaced by the amount $\Delta L$ in the convergent section of the nozzle, as sketched in Fig. 5.

Purely longitudinal modes may be constructed by superposing leftwardand rightward-traveling waves. The amplitudes are constant in a uniform section but suffer abrupt changes at a discontinuity of area. At a discontinuity, the acoustic pressure and mass flux must be continuous. The boundary conditions are that the axial velocity must vanish at the forward and aft ends, $z=0, L$. Within the $j$ th uniform section, we represent the acoustic pressure and velocity as

$$
\begin{aligned}
\frac{p_{j}^{\prime}}{\gamma \bar{p}} & =\left[P_{i}^{+} e^{-i k z}+P_{j}^{-} e^{i k z}\right] e^{i \omega t} \\
\frac{u_{j}^{\prime}}{\bar{a}} & =\left[P_{i}^{+} e^{-i k z}-P_{j}^{-} e^{i k z}\right] e^{i \omega t}
\end{aligned}
$$

where the superscripts denote rightward ()$^{+}$and leftward ()$^{-}$traveling waves. The coefficients $P_{j}^{+},-P_{j}^{--}$in the formula for the velocity are set by satisfying the unperturbed acoustic momentum equation.

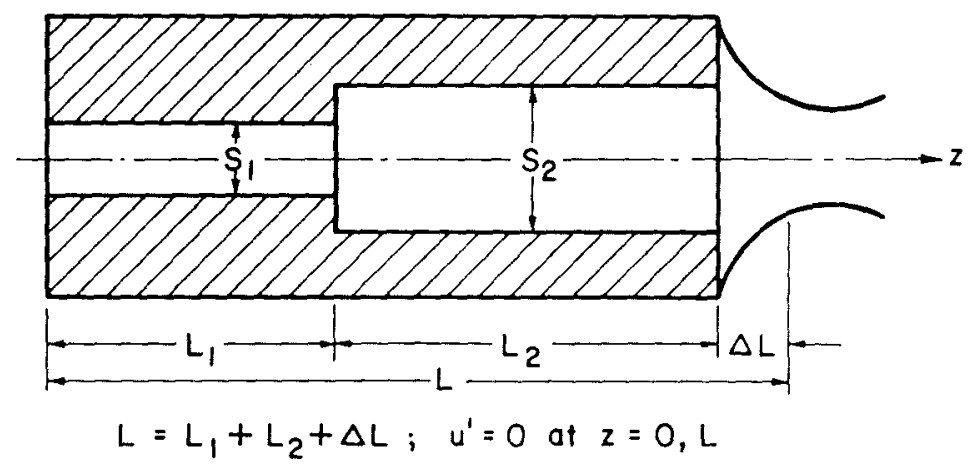

Fig. 5 Schematic of a rocket motor with two segments of propellant grains. 
To satisfy $u_{1}^{\prime}=0$ at $z=0, P_{1}^{-}=P_{1}^{+}$in Sec. I, and to meet the condition $u_{2}^{\prime}=0$ at $z=L, P_{2}^{-}=P_{2}^{+} e^{-i 2 k L}$ in Sec. II. Continuity of pressure at $z=L_{1}$ requires

$$
P_{1}^{+}\left[e^{-i k L_{1}}+e^{i k L_{1}}\right]=P_{2}^{+}\left[e^{-i k L_{1}}+e^{i k\left(L_{1}-2 L\right)}\right]
$$

For continuity of acoustic momentum at $z=L_{1}, u_{1}^{\prime} S_{1}=u_{2}^{\prime} S_{2}$ because the average density is uniform. This condition is met if

$$
P_{1}^{+}\left[e^{-i k L_{1}}-e^{i k L_{1}}\right] S_{1}=P_{2}^{+}\left[e^{-i k L_{1}}-e^{i k\left(L_{1}-2 L\right)}\right] S_{2}
$$

Equations (137) and (138) have nontrivial solutions for $P_{1}^{+}$and $P_{2}^{-}$if

$$
\frac{1+e^{i 2 k L_{1}}}{1-e^{i 2 k L_{1}}}=\frac{S_{1}}{S_{2}} \frac{1+e^{i 2 k\left(L_{1}-L\right)}}{1-e^{i 2 k\left(L_{1}-L\right)}}
$$

This is the equation for determining the dimensionless wave number $k L$ as a function of the two parameters $L_{1} / L$ and $S_{2} / S_{1}$.

Some numerical results for $k L / \pi$ are shown in Fig. 6 . Note that these have been computed for the fundamental mode: $k L=\pi$ for all $L_{1} / L$ when $S_{2}=S_{1}$. On physical grounds, the value of $k L$ for particular values $L_{1} / L$ $=\lambda, S_{1} / S_{2}=\sigma$ must also be obtained for $L_{1} / L=1-\lambda$ and $S_{1} / S_{2}=$ $1 / \sigma$. That constraint helps explain some of the behavior shown by the curves. Similar plots can of course be prepared for higher modes. This example has been chosen to be a simple illustration. The idea has been elaborated in more detail for, among other things, application to Tburners. ${ }^{43,45}$

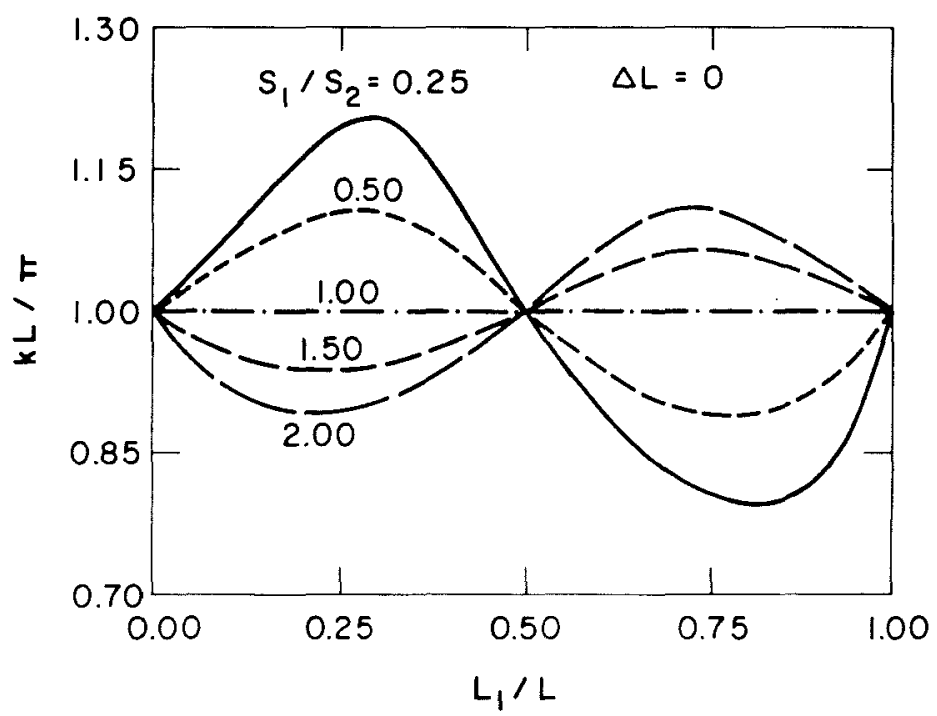

Fig. 6 Acoustic wave number of the fundamental mode. 
It is equally easy to show the influence of the exhaust nozzle. Now we consider a uniform chamber. Suppose that the head end admits flow through a choked porous plate that imposes the boundary condition $u^{\prime}=0$ at $z=0$. The entrance of the nozzle is assumed to fair smoothly to the chamber, as shown in Fig. 7, and sets a boundary condition expressed in terms of its admittance function, $A_{N}=\left(u^{\prime} / \bar{a}\right) /\left(p^{\prime} / \gamma \bar{p}\right)$ at $z=L$. We assume that the flow is uniform in the chamber, with Mach number $\bar{M}$. Then a stationary longitudinal mode can again be represented as the sum of rightward- and leftward-moving waves:

$$
\begin{aligned}
& \frac{p^{\prime}}{\gamma \bar{p}}=\left[P^{+} e^{-i K z}+P^{-} e^{i K z}\right] e^{i(\Omega t+\bar{M} K z)} \\
& \frac{u^{\prime}}{\bar{a}}=\left[P^{+} e^{-i K z}-P^{-} e^{i K z}\right] e^{i(\Omega t+\bar{M} K z)}
\end{aligned}
$$

Satisfaction of the boundary conditions at $z=0, L$ again gives two equations for $P^{+}$and $P^{-}$. Nontrivial solutions exist if the wave number satisfies the equation

$$
\frac{1-e^{i 2 K L}}{1+e^{i 2 k L}}=A_{N}
$$

where

$$
K=\frac{k}{1-\bar{M}^{2}}=\frac{1}{\bar{a}} \frac{\omega-i \alpha}{1-\bar{M}^{2}}
$$

Because $A_{N}$ is complex, so too is $K$.

The result Eq. (141) must be solved numerically, giving exact results affording useful comparison with the approximate results found earlier. With only the contribution of the nozzle retained, Eq. (97) gives the frequency shift

$$
\omega-\omega_{n}=-\frac{\bar{a}^{2}}{2 \omega_{n} \bar{p} E_{n}^{2}} \bar{p} k_{n} \iint A_{N}^{(i)} \psi_{n}^{2} \mathrm{~d} S
$$

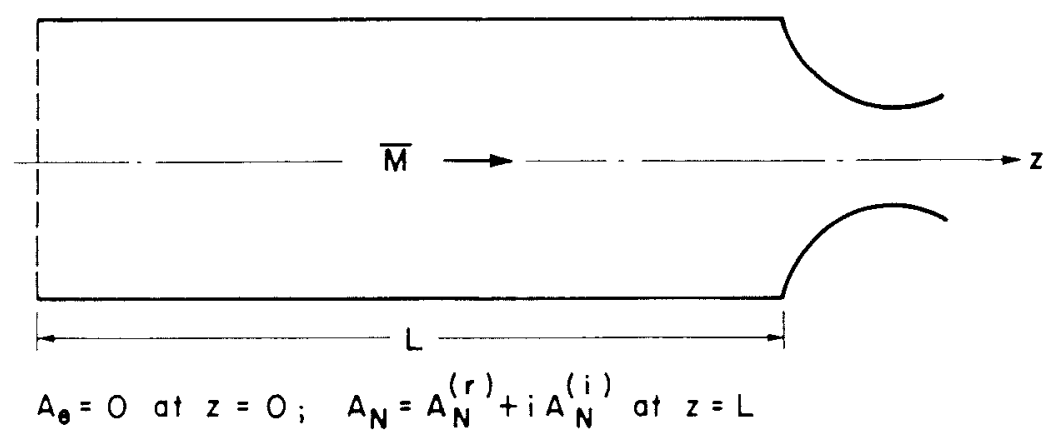

Fig. 7 Schematic of a combustion chamber with uniform mean flow. 
For a straight tube, $\psi_{n}=\cos (n \pi z / L)$, giving $E_{n}^{2}=S_{N} L / 2$, and for $A_{N}$ uniform over the nozzle entrance,

$$
\omega_{a}-\omega_{n}=-\frac{\bar{a}}{L} A_{N}^{(i)}
$$

where subscript $a$ shows this to be an approximate value.

Equation (100) contains only those terms associated with the nozzle and entrance plane,

$$
\alpha_{N}=-\frac{\bar{a}}{2 E_{n}^{2}} \iint_{\text {Nozzle }}\left[A_{N}^{(r)}+\bar{M}_{N}\right] \psi_{n}^{2} \mathrm{~d} S-\frac{\bar{a}}{2 E_{n}^{2}} \iint_{\text {entrance }} \bar{M}_{e} \psi_{n}^{2} \mathrm{~d} S
$$

We assume that the admittance is zero at the head end. Again, $E_{n}^{2}=S_{N} L /$ 2 ; the flow is inward at the entrance, and so the terms containing the Mach number cancel and we find, for the decay constant,

$$
\alpha_{a}=-\frac{\bar{a}}{L} A_{N}^{(r)}
$$

Figures 8 and 9 show comparisons of Eqs. (143) and (144), with the corresponding results computed with Eq. (141). The error accompanying the approximations is less than about $5 \%$ for Mach numbers less than 0.20 . This is confirmation, with an elementary example, of course, that indeed the approximate analysis is very effective.

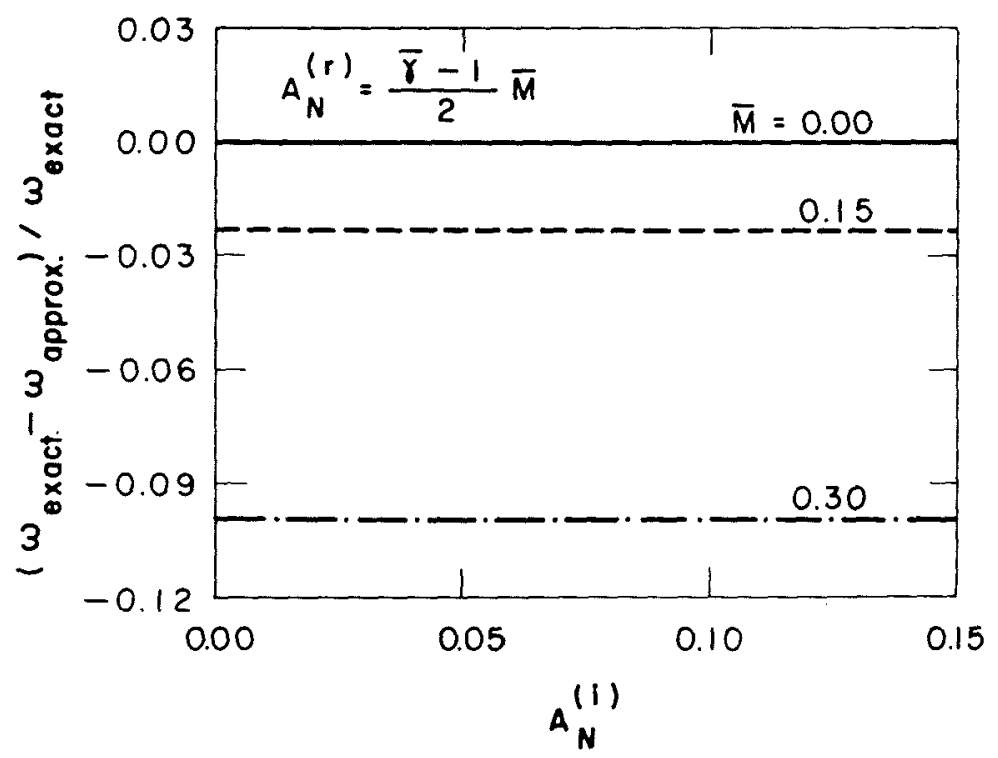

Fig. 8 Dependence of frequency on the imaginary part of acoustic nozzle admittance function. 


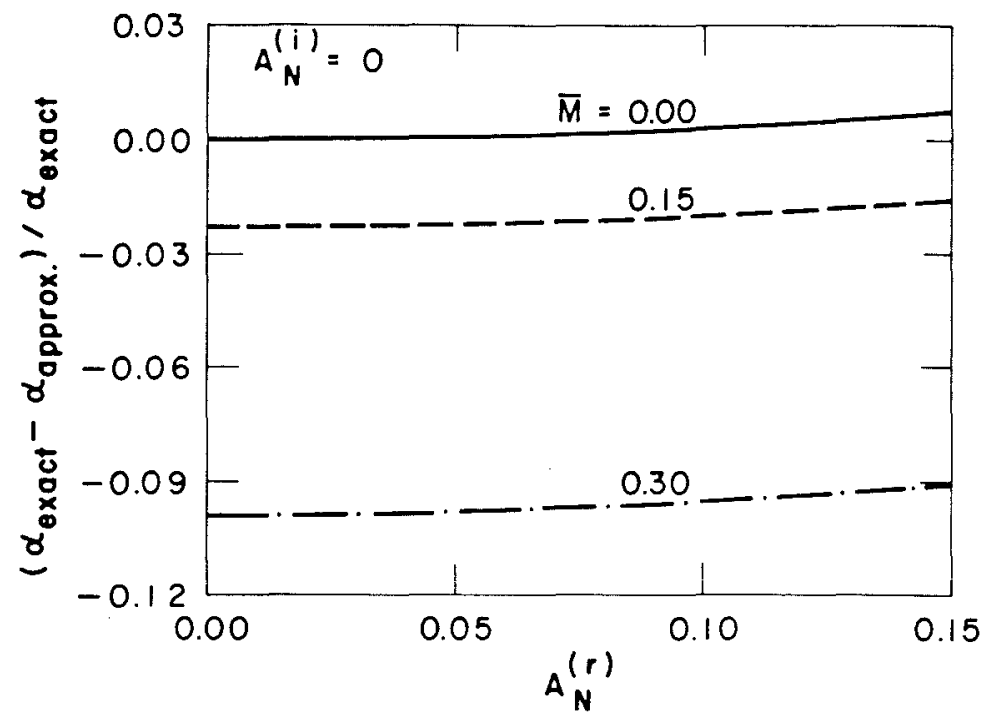

Fig. 9 Dependence of growth constant on the real part of acoustic nozzle admittance function.

\section{Some Elementary Aspects of Nonlinear Behavior}

We emphasized in the Introduction that combustion instabilities are a likely occurrence in solid-propellant rockets. Linear analysis provides a basis for assessing whether a particular system is unstable. The primary result is that small-amplitude motions are stable if and only if the losses of acoustic energy dominate the gains. If that is not true, then the exponential growth rate is a measure of the excess of gains over losses.

However, linear theory provides no information about the ultimate amplitudes of unstable motions. As far as these disturbances are concerned, the combustion chamber appears as a self-excited system. An unstable motion will settle down to a stable limiting amplitude only through the action of nonlinear processes. Given the circumstance that instabilities are likely, it is therefore necessary to pay attention to nonlinear behavior. For practical purposes, the objective is to understand what controllable parameters determine the limiting amplitudes of unstable motions.

There are actually two classes of nonlinear problems to be studied: 1) the conditions for existence and stability of finite oscillatory motions or limit cycles in a linearly unstable system; and 2) the form (e.g., amplitude and spectral content) of the initial disturbance that will cause a linearly stable system to execute limit cycles, a phenomenon sometimes called "triggering." Here we shall consider only the first sort of problem.

General nonlinear behavior is an active area of research. Here, to simplify matters, we treat only the case for purely longitudinal modes in a chamber having a rigid head end and a choked exhaust nozzle. Then the 
classical unperturbed modes are those for a closed-closed chamber, having frequencies that are integral multiples of the fundamental, $\omega_{n}=n \omega_{1}$. Also, the nonlinearities are carried only to second order in the fluctuations. The gasdynamic nonlinearities to this order are shown explicitly in the functions $h$ and $f$, Eqs. (40) and (42). Because there are really two parameters characterizing the gasdynamic motions - the Mach numbers of the average and fluctuating flows - there is no unique set of nonlinear equations, a fact that becomes apparent when the expansion is carried to third order. We must then decide, for example, whether terms of order $\bar{M}\left(M^{\prime}\right)^{2}$ are of the same order as those of order $\left(M^{\prime}\right)^{3}$. Such questions, as well as possible difficulties in analysis of motions not purely longitudinal, lie outside the scope of this discussion. We shall ignore all other nonlinear processes, although the framework developed in Sec. III will accommodate any nonlinear contribution arising in practice.

The nonlinear terms in the functions $h$ and $f$, Eqs. (40) and (42), must now be retained. After the expansions (45) and (46) have been substituted, the forcing function $F_{n}$, defined by Eq. (49) becomes a nonlinear function of the amplitudes $\eta_{n}$. Hence, Eq. (48) represents a set of coupled nonlinear oscillators. Because only terms of second order have been retained, here, in $h$ and $f$, the equation for $\eta_{n}$ is only of second order, with $F_{n}$ given explicitly by Eq. (75).

In general, the coupled equations are further complicated because $F_{n}$ contains a double series. A great simplification accompanies the assumption of purely longitudinal modes; for then the double series becomes a single series. After time averaging, the equations for $A_{n}(t)$ and $B_{n}(t)$ are ${ }^{11}$

$$
\begin{aligned}
& \frac{\mathrm{d} A_{n}}{\mathrm{~d} t}=\alpha_{n} A_{n}+\theta_{n} B_{n}+\frac{\beta n}{2} \sum_{i=1}^{\infty}\left[A_{i}\left(A_{n-i}-A_{i-n}-A_{i+n}\right)\right. \\
& \left.\quad-B_{i}\left(B_{n-i}+B_{i-n}+B_{i+n}\right)\right] \\
& \frac{\mathrm{d} B_{n}}{\mathrm{~d} t}=\alpha_{n} B_{n}-\theta_{n} A_{n}+\frac{\beta n}{2} \sum_{i=1}^{\infty}\left[A _ { i } \left(B_{n-i}\right.\right. \\
& \left.\left.\quad+B_{i-n}-B_{i+n}\right)+B_{i}\left(A_{n-i}-A_{i-n}+A_{i+n}\right)\right]
\end{aligned}
$$

For $N$ modes, there are $2 N$ equations to be solved; a simple transformation of the dependent variables reduces the number to $2 N-1$, a consequence of the freedom to set one phase arbitrarily. ${ }^{46}$ Thus, for two modes, there are only three equations, and exact solution can be obtained for the limiting amplitudes forming the limit cycle. The same results were found previously with a different calculation by Awad and Culick. ${ }^{47}$ It is apparent from Eqs. (145a) and (145b) that the only nonlinear parameter, $\beta$, arises as a scaling parameter; the values of the limiting amplitudes depend only on the linear 
parameters $\left(\alpha_{n}, \theta_{n}\right)$ and are given by the formulas

$$
\begin{aligned}
& r_{1}=\frac{1}{\beta}\left\{-\alpha_{1} \alpha_{2}\left[1+\left(\frac{2 \theta_{1}-\theta_{2}}{2 \alpha_{1}+\alpha_{2}}\right)^{2}\right]\right\}^{1 / 2} \\
& r_{2}=\frac{\alpha_{1}}{\beta}\left\{1+\left(\frac{2 \theta_{1}-\theta_{2}}{2 \alpha_{1}+\alpha_{2}}\right)^{2}\right\}^{1 / 2}
\end{aligned}
$$

where $r_{n}=\left(A_{n}^{2}+B_{n}^{2}\right)^{1 / 2}$.

For a limit cycle to exist, $\alpha_{1} \alpha_{2}$ must be negative; that is, when the oscillations are steady, the energy in the motion is constant, so that one mode gains energy from the environment and the other loses energy. Nonlinear processes associated with the gasdynamics have a natural tendency to cause energy flow from lower to higher modes. Thus, in general, smoother transient growth of the oscillation occurs when the first mode is unstable $\left(\alpha_{1}>0\right)$ and the second is stable $\left(\alpha_{2}<0\right)$.

This formulation of nonlinear acoustics seems to contain much of the behavior observed in practice and, under the conditions for which it is valid, accuracy is high. Examples are discussed in the following section. It is beyond the intent of this chapter to treat nonlinear problems in greater detail. Nonlinear behavior of combustion instabilities is an important practical and theoretical matter that remains a subject of current research.

\section{Numerical Analysis of Combustion Instabilities}

So far, we have examined various aspects of linear and nonlinear combustion instabilities in solid-propellant rocket motors as well as a method of approximate solution. Although these analytical methods provide direct and economical means of assessing the influence of each contributing mechanism in a formal and systematic manner, they sometimes suffer from lengthy formulation and cannot represent with full precision the detailed physicochemical processes involved. This is particularly true for cases involving complicated geometry, steep-fronted waves, and nonlinear combustion response. On the other hand, numerical analysis is intended to obtain the "exact" solution to the set of conservation equations [Eqs. (710)], subject to the appropriate initial and boundary conditions. The results can therefore be used to check the accuracy of the approximate analysis, which is more conveniently used for routine calculations and for formal theoretical work. Thus, a complete treatment of motor stability characteristics should include both analytical and numerical approaches. In this section, a brief review of the numerical calculations of longitudinal oscillations is given.

Several studies (Refs. 48-51) have been devoted to the numerical analysis of longitudinal combustion instabilities in a solid-propellant rocket motor. All of the formulations in these studies are based on the unsteady one-dimensional equations of motion for a two-phase flow. Levine and Culick $^{48}$ appear to have been the first to treat a problem of this sort, using the method of characteristics. The purposes of the original works were to assess the feasibility of obtaining complete nonlinear numerical solutions 
to longitudinal instability problems and to compare the results with those of the approximate analysis. Therefore, the model was deliberately kept simple, with linear analysis of the transient propellant burning rate response. After successfully demonstrating several salient characteristics of a nonlinear system, in particular, the existence of limit cycles, some of the assumptions and restrictions of the model were relaxed in order to establish an analytical capability to analyze or predict the stability behavior of a wide range of solid rocket motor configurations. The analysis then progressed in several areas. ${ }^{49}$ First, for reasons of computational efficiency and accuracy, a finite difference technique was adopted in place of the method of characteristics. Second, more complete treatments of particle size distribution, nozzle flow, and nonlinear combustion response were included. Third, in order to accommodate more general and realistic grain geometry (such as variable cross-sectional area and arbitrary cross-sectional shapes) within the structure of the instability model, the equations of motion and boundary conditions were generalized. Thus, with an appropriate treatment of the unsteady burning mechanisms of propellants, the analysis offers the potential to predict motor instability behavior. As part of the verification, a series of motor instability calculations was carried out and compared with experimental data obtained with small laboratory pulse motors. The influences of pulse strength and propellant combustion response on the limiting amplitude of pressure oscillations have been examined at considerable length.

The analysis developed by Levine and Culick $^{49}$ provides a firm basis for studying longitudinal combustion instabilities and has been successfully applied to other related problems, including nonlinear particle damping and nozzle flows. However, as a result of the numerical scheme used, the program was incapable of treating pulse-triggered combustion instabilities, or the multiple-shock, steep-fronted type of instabilities that often occur in reduced and minimum-smoke tactical motors with variable cross-sectional area. In order to study these problems faithfully, a suitable numerical scheme must be developed to preserve the complete spectral information of the waveforms, minimize the numerical dissipative and dispersive errors, accurately describe shock and rarefraction waves without generating nonphysical oscillations in the vicinities of discontinuities, and properly treat the wave reflection and transmission at area discontinuities. For quasi-onedimensional longitudinal instabilities, the self-adjusting hybrid scheme with an artificial compression correction ${ }^{52,53}$ appears to be the best option to fulfill these requirements, and has been incorporated in improvements of the original nonlinear analysis. ${ }^{51}$ The scheme consists of a second-order Lax-Wendroff scheme and a hybrid operator that switches automatically and smoothly to a first-order approximation in narrow shock regions, in accordance with the local density gradient. It therefore possesses the merits of both first- and second-order schemes, yielding nonoscillatory shock transitions and accurate results in smooth regions. The spurious pre- and postshock oscillations produced by second-order finite difference approximations can be completely eliminated.

This approach has proved robust and can treat the propagation of a traveling shock wave over many wave cycles without introducing significant 
diffusive or dispersive errors. Because of its remarkable shock-capturing feature, the hybrid scheme has been widely used by Levine and Baum in their study of pulse-triggered combustion instabilities. ${ }^{54-56}$ Later, Levine et al. ${ }^{27}$ also employed this program to calculate the nonlinear particle damping produced by traveling longitudinal shock waves in a simulated motor environment.

Although the one-dimensional analysis developed by Baum and Levine ${ }^{51}$ appears to accommodate most of the essential features of nonlinear longitudinal combustion instabilities (e.g., waveform evolution, growth and decay rate, dc shift, limiting cycles), it requires empirical adjustment of the velocity-coupled combustion response to obtain the best possible agreement with the experimental observations. To enhance the basic understanding of the problem and therefore improve the predictive capability of the analysis, further research is required on the multidimensional interactions of propellant combustion response and the oscillatory flowfields in a motor environment, since velocity coupling is a multidimensional local phenomenon that depends on mean flow properties, characteristics of flow oscillations, and thermochemical properties of propellants. The conventional one-dimensional approach does not provide meaningful results for this subject.

To date, multidimensional numerical analysis of motor instability has not been worked out although most of the groundwork has been set. The major difficulty lies in the lack of computational capability and the modeling of various physical mechanisms. With the exception of the works by Yang et al. ${ }^{15}$ and Vuillot and Avalon ${ }^{62}$ for studying the velocity-coupled flow oscillations in a simulated rocket motor environment, most of the numerical efforts have been limited to investigation of internal flowfields under steady-state conditions. ${ }^{57,58}$ Yang and his co-workers considered a case involving a two-dimensional porous chamber with air injected through the walls to simulate the evolution of combustion products of solid propellants. The theoretical model is based on the full time-dependent compressible Navier-Stokes equations and is solved numerically by means of finite difference algorithms. The results indicate that the multidimensional effects play important roles in determining oscillatory flow characteristics. The waveform and phase distribution of acoustic oscillations depend greatly on the local flow structures. The classical one-dimensional theory failed to describe several important mechanisms associated with acoustic waveinduced flow instabilities. Extension of the work to cases including burning propellants is given in Ref. 63 .

It is inappropriate here to reproduce the details of the numerical analysis, which is thoroughly discussed in the references cited. However, to complete this brief survey of methods for predicting combustion instabilities, it is useful to give the results of an example illustrating comparison with the approximate analysis. The example chosen is a straight cylindrical motor with port fairing smoothly into a choked nozzle. Values of the geometrical and physical properties are listed in the Appendix. The conditions have been chosen so as to cause the fundamental mode to be unstable; all higher modes are stable. 
Numerical analysis of the transient development of the motion following an initial disturbance was computed with the computer program developed by Baum and Levine, as reported in Ref. 51 . Two cases have been treated: with and without flow-turning losses included. The initial disturbance has the form of the fundamental classical mode of the chamber, having an amplitude $5 \%$ of the mean pressure. Figure 10 shows that the unstable oscillations eventually reached a limiting amplitude of about $15 \%$ of the mean pressure, computed for the case in which flow-turning losses are absent. The waveform, of course, contains all normal modes of the chamber. However, the Fourier analysis of the limiting waveform (Fig. 11) confirms that the amplitudes of the lowest modes dominate.

For comparison, the same problem has been solved with the approximate analysis. Solution of Eqs. (145a) and (145b) requires calculation of the classical mode shapes and frequencies; then the values of the linear parameters $\left(\alpha_{n}, \theta_{n}\right)$ can be determined by summing the contributions from the various physical processes. Only two modes will be considered. For the straight cylindrical chamber considered here, $\psi_{n}=\cos (n \pi z / L)$, and the calculations are easily done. We assume that the response function for the burning solid is constant. Equations (102) and (97) then give

$$
\begin{aligned}
& \alpha_{C}=\frac{\bar{u}_{b}}{r_{c}} R_{b}^{(r)} \\
& \theta_{C}=-\frac{\bar{u}_{b}}{r_{c}} R_{b}^{(i)}=-\frac{R_{b}^{(i)}}{R_{b}^{(r)}} \alpha_{c}
\end{aligned}
$$

where $\theta_{C}=-\left(\omega-\omega_{n}\right)$, as defined in Eq. (77b). The combustion response is calculated using the approximate result obtained by Denison and Baum ${ }^{18}$

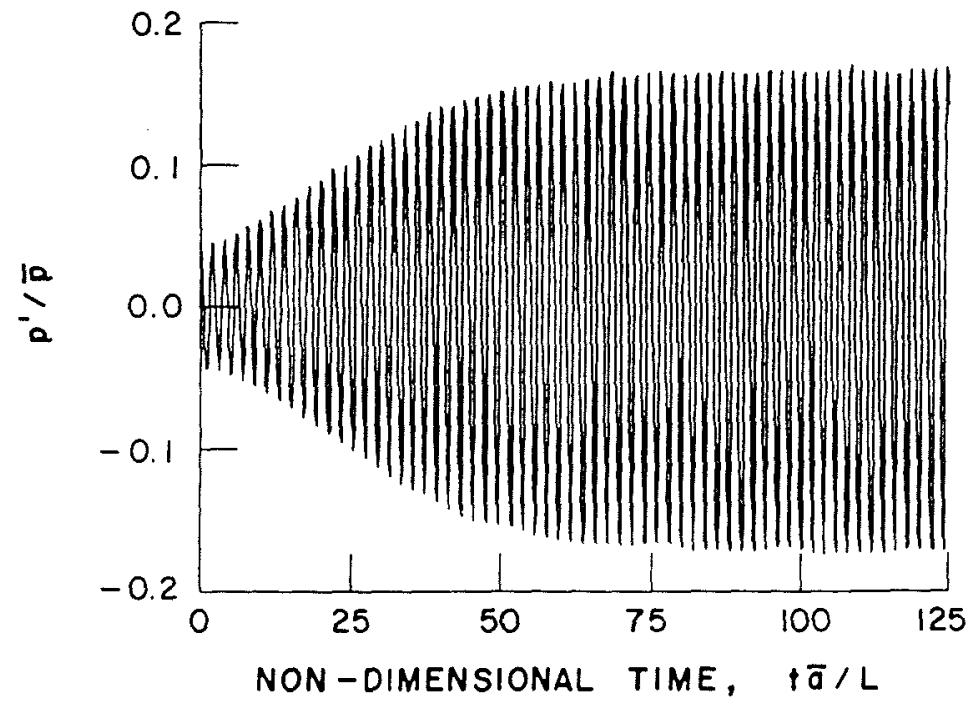

Fig. 10 Time history of acoustic pressure based on the numerical analysis. 


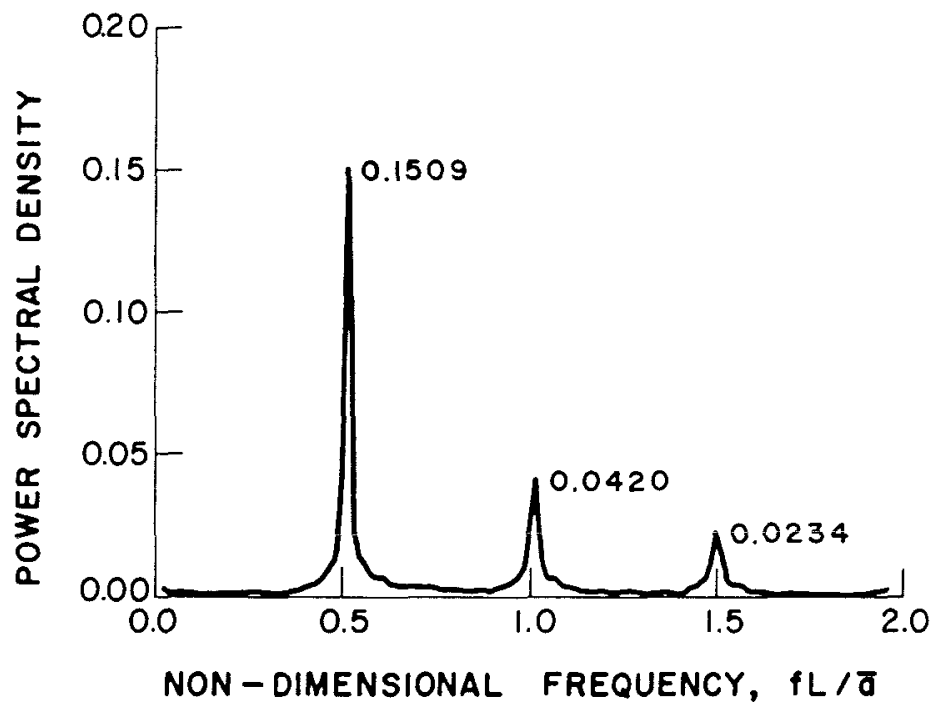

Fig. 11 Power spectral density of acoustic pressure.

and reviewed by Culick $^{19}$; the numerical values used here are shown in Fig. 1.

We assume that the area of the entrance to the nozzle equals the port area and that the nozzle responds quasistatically, so that its admittance function is real: $A_{N}^{(r)}=(\bar{\gamma}-1) \bar{M}_{N} / 2$ and $A_{N}^{(i)}=0 .{ }^{59,60}$ Hence, $\theta_{N}=0$, according to Eq. (97), and Eq. (100) gives

$$
\alpha_{N}=-\frac{\bar{a}}{L}\left(\frac{\bar{\gamma}+1}{2}\right) \bar{M}_{N}
$$

Condensed aluminum oxide is distributed uniformly in the combustion gases, with uniform average particle size. For longitudinal modes, the formulas (125a) and (125b) become

$$
\begin{aligned}
\alpha_{P} & =-\frac{\omega_{n}}{2} \frac{C_{m}}{1+C_{m}}\left[\frac{\Omega_{d}}{1+\Omega_{d}^{2}}+(\bar{\gamma}-1) \frac{C}{\bar{C}_{p}} \frac{\Omega_{t}}{1+\Omega_{t}^{2}}\right] \\
\theta_{P} & =\frac{\omega_{n}}{2} \frac{C_{m}}{1+C_{m}}\left[\frac{\Omega_{d}^{2}}{1+\Omega_{d}^{2}}+(\bar{\gamma}-1) \frac{C}{\bar{C}_{p}} \frac{\Omega_{t}^{2}}{1+\Omega_{t}^{2}}\right]
\end{aligned}
$$

With the data given in the Appendix, we find in Table 1 the values for $\alpha_{n}$ and $\theta_{n}$ characterizing the first five modes in the absence of flow-turning losses. All values of $\left(\alpha_{n}, \theta_{n}\right)$ have units $\mathrm{s}^{-1}$. The total values are listed in Table 2.

Solutions have been obtained for the transient behavior following an initial disturbance having the spatial distribution of the first mode: $B_{1}(0)$ 
Table 1 Linear growth constants and frequency shifts

\begin{tabular}{ccccccr}
\hline Mode & $\alpha_{c}$ & $\theta_{c}$ & $\alpha_{N}$ & $\theta_{N}$ & $\alpha_{P}$ & $\theta_{P}$ \\
\hline 1 & 288.1 & 32.2 & -160.1 & 0 & -46.6 & 2.6 \\
2 & 28.5 & 80.5 & -160.1 & 0 & -184.8 & 20.5 \\
3 & 16.7 & 48.5 & -160.1 & 0 & -417.7 & 69.1 \\
4 & 13.6 & 36.0 & -160.1 & 0 & -727.4 & 160.5 \\
5 & 12.0 & 29.3 & -160.1 & 0 & -1107.5 & 305.5 \\
\hline
\end{tabular}

$=0.05$, and all other values of $A_{n}(0), B_{n}(0)$ are zero, so that

$$
\begin{aligned}
& p^{\prime}(z, 0)=0.05 \bar{p} \cos (\pi z / L) \\
& u^{\prime}(z, 0)=0
\end{aligned}
$$

Figure 12 shows the evolution of the oscillation for comparison with Fig. 10, and Fig. 13 shows the growth of the amplitudes $r_{n}=\left(A_{n}^{2}+B_{n}^{2}\right)^{1 / 2}$ for the first five modes, found as the solutions to Eqs. (145a) and (145b) with $n=1$ through 5 .

For direct comparison of the numerical and approximate results, Table 3 contains the values of the frequencies and amplitudes computed for the first two modes. A few cycles of the limiting waveforms are plotted in Fig. 14.

Note that the approximate analysis produces quite accurate values of the frequencies (within 3\%) and that the approximate total waveform is reasonably close to the "exact" result except for some rippling due to the absence of higher frequencies. Even so, the amplitudes of the individual modes found with the approximate analysis agree well with those computed with the numerical analysis except for the highest $(n=5)$ mode. The reason for the high value (even larger than that for $n=4$ ) is that there is no transfer of energy to higher modes. Because the rate of energy dissipation from the highest mode considered, represented by its attenuation constant, must be such that the total energy loss equals the total rate of energy gain, the amplitude of the highest mode must be such as to satisfy this condition. Even with only two modes accounted for, the frequencies

Table 2 Total values of linear growth constants and frequency shifts

\begin{tabular}{rrr}
\hline Mode & \multicolumn{1}{c}{$\alpha_{n}$} & \multicolumn{1}{c}{$\theta_{n}$} \\
\hline 1 & 81.4 & 34.8 \\
2 & -316.4 & 100.9 \\
3 & -561.1 & 117.6 \\
4 & -873.9 & 196.6 \\
5 & -1255.6 & 334.8 \\
\hline
\end{tabular}




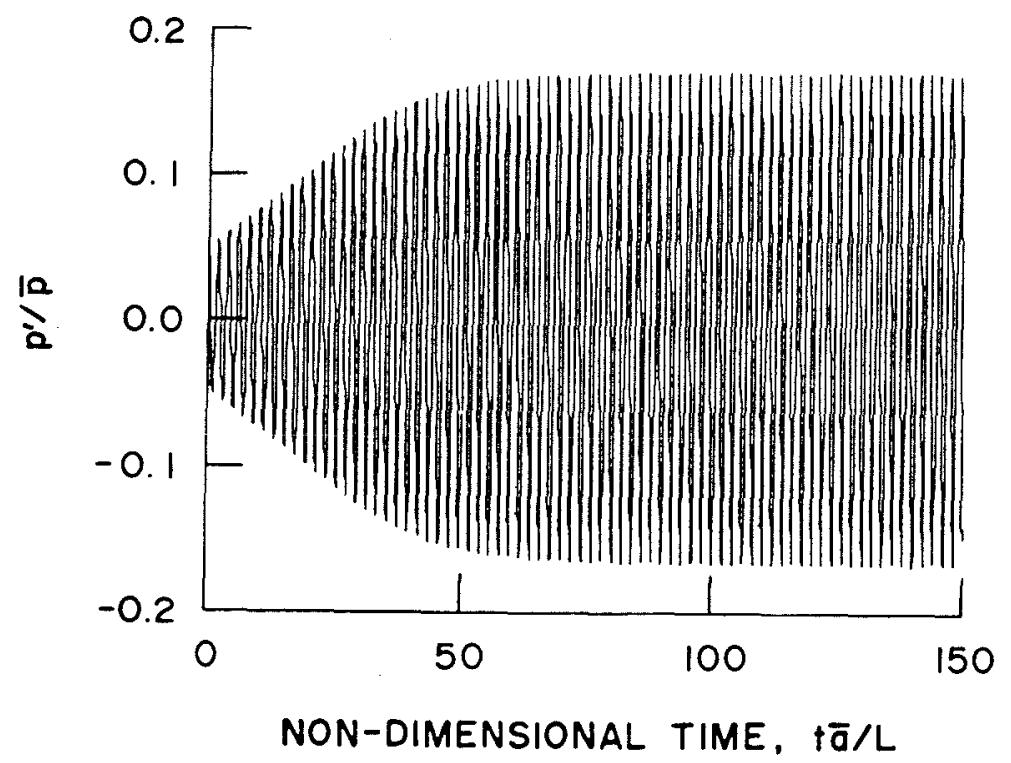

Fig. 12 Time history of acoustic pressure based on the approximate analysis.

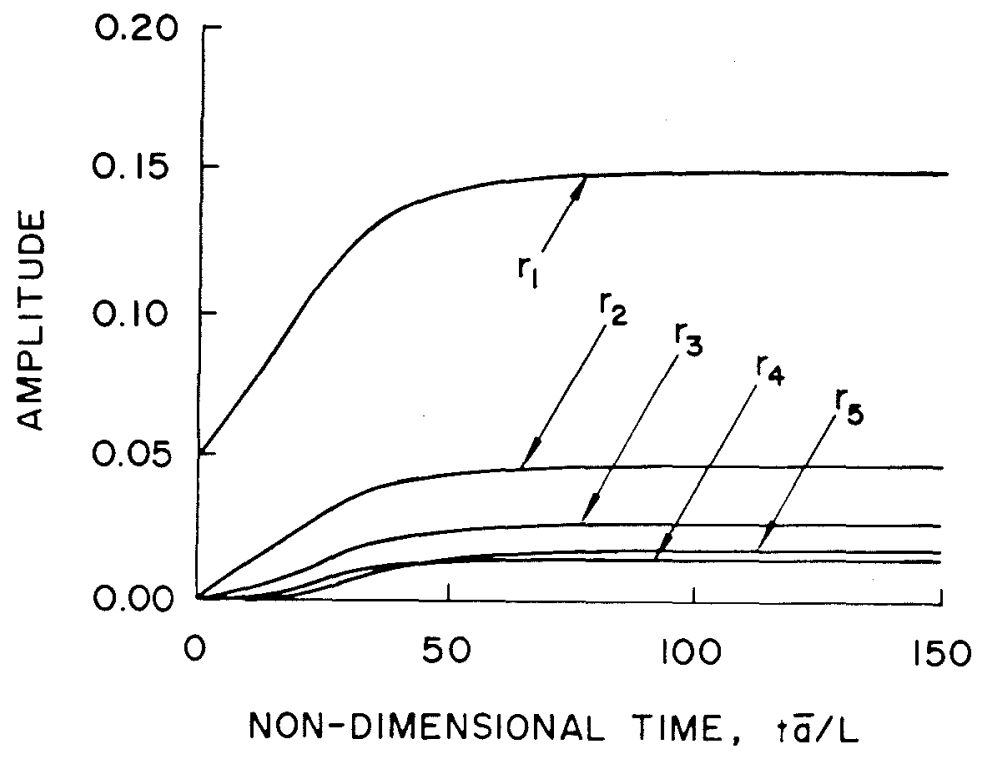

Fig. 13 Time histories of acoustic pressure amplitudes. 


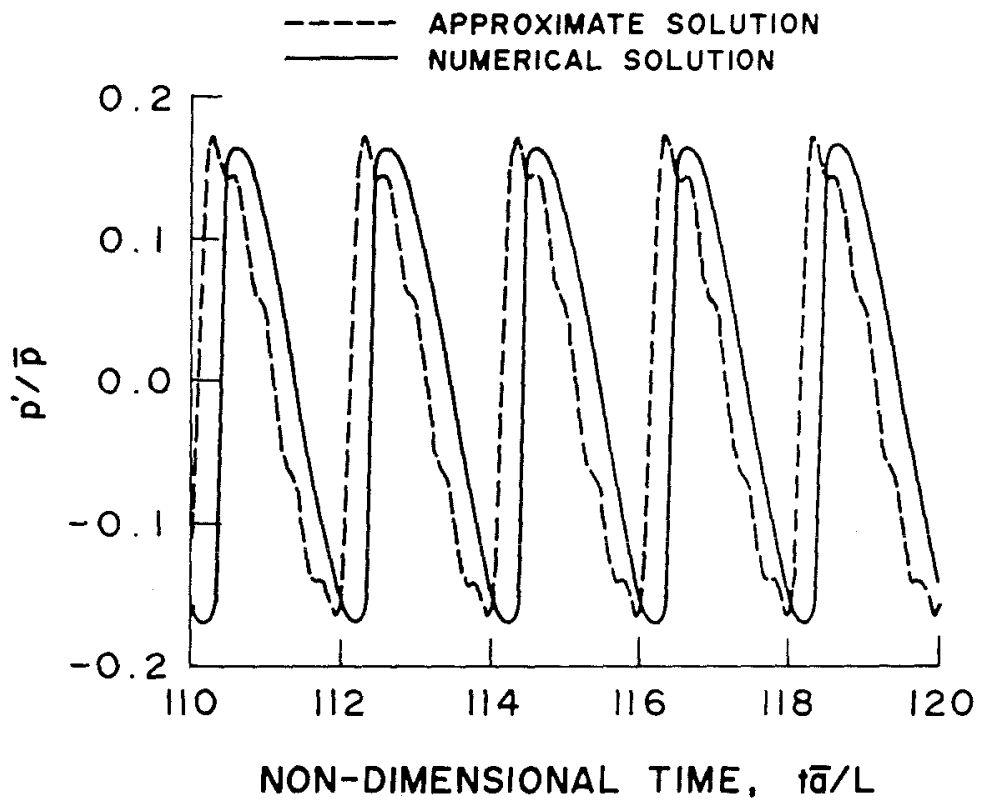

Fig. 14 Comparison of calculated acoustic pressures based on the numerical and approximate analyses.

and total waveform are quite well predicted. The frequencies are the same as for the case of five modes; Fig. 15 shows the waveform: However, the approximate amplitudes of the individual modes differ considerably from the exact values. Apparently, the phases of the two modes in the approximate solution assume values that compensate for the consequences of the inaccurate amplitudes.

Some preliminary results have been computed for essentially the same problem but with flow-turning losses accounted for. For reasons that are not known at this time, the limiting behavior predicted with the approximate analysis is markedly different from that found numerically. The frequencies are as close as those found earlier, but the approximate amplitudes are only about half those of the "exact" values for the case in which only two modes are included. The restriction to two modes may be an important reason for the discrepancy. The consequences of this assumption are dif-

$$
4
$$

Table $\mathcal{\beta}$ Frequencies and amplitudes of acoustic pressures

\begin{tabular}{|c|c|c|c|c|c|c|c|c|c|c|}
\hline \multirow[b]{2}{*}{ Mode } & \multicolumn{5}{|c|}{ Frequency, $\mathrm{Hz}$} & \multicolumn{5}{|c|}{ Amplitude, $\left|p^{\prime} / \bar{p}\right|$} \\
\hline & 1 & 2 & 3 & 4 & 5 & 1 & 2 & 3 & 4 & 5 \\
\hline $\begin{array}{l}\text { Numerical } \\
\text { Approximate }\end{array}$ & $\begin{array}{l}926 \\
895\end{array}$ & $\begin{array}{l}1824 \\
1785\end{array}$ & $\begin{array}{l}2698 \\
2683\end{array}$ & $\begin{array}{l}3595 \\
3571\end{array}$ & $\begin{array}{l}4491 \\
4449\end{array}$ & 0.151 & 0.042 & 0.0234 & 0.0203 & $\overline{0.0188}$ \\
\hline
\end{tabular}




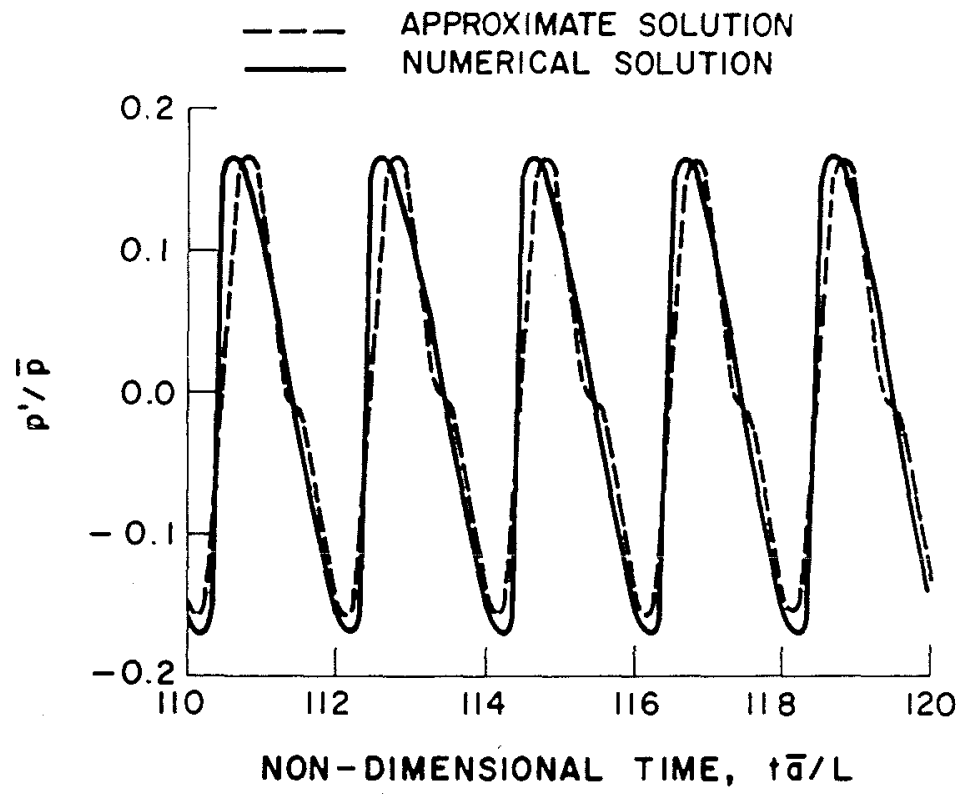

Fig. 15 Comparison of calculated acoustic pressures based on the numerical and approximate analyses.

ferent when flow turning is included because this contribution to the losses is independent of frequency $\left(-73.5 \mathrm{~s}^{-1}\right.$ for this problem). In contrast, the loss due to particle damping increases rapidly with frequency. Hence, for an accurate comparison, it may be necessary to account for more modes in the approximate analysis. The problem is a subject of continuing investigation.

The influence of the flow-turning losses is an important matter to clarify. Its contribution to the decay constant is often large, but its value is known only by analysis based on the one-dimensional approximation to the unsteady motions. Here the result for a cylindrical grain is obtained from the last term of Eq. (135):

$$
\alpha_{F T}=-\frac{\bar{u}_{b}}{r_{c}}=-\frac{\bar{a}}{r_{c}} \bar{M}_{b}
$$

Neither computational nor experimental methods have yet established the accuracy of this formula.

\section{Concluding Remarks}

The approximate analysis covered in this chapter seems to provide satisfactory results for linear stability. It is not possible to place any general bounds on expected inaccuracies for two reasons: uncertainties in the required input data depend on the case considered, and comparisons with experimental results are scarce and always contain errors difficult to assess. 
The greatest obstacle to improving this situation is obtaining good data for the propellant response function.

Comparison with "exact" numerical solutions to the complete conservation equations is the only way to determine the truth of the approximate predictions for nonlinear behavior. Although only limited results have been obtained, it appears that nonlinear gasdynamics is commonly the process most influential in limiting the amplitudes of combustion instabilities involving longitudinal modes. The approximate analysis has been applied elsewhere to transverse modes of oscillation, giving formal results corresponding to those discussed here. However, no nonlinear numerical analysis is currently available to treat those problems, and so no comparisons exist.

\section{Appendix}

The numerical values of the quantities required in the example discussed in Sec. VI are listed in the following.

Geometrical properties:

length

radius of cylindrical port

throat radius

Combustion properties:

mean pressure

linear burning rate

parameters in the

combustion response

chamber temperature

mass particles/mass gas

particle diameter

Physical properties:

Prandtl number

thermal diffusivity of

propellant

specific heat of gas

specific heat of condensed

material

viscosity

particle density

propellant density

gas density

$\gamma$ (gas only)

$\bar{\gamma}$ (mixture)

gas constant
$L=0.5969 \mathrm{~m}$

$r_{c}=0.0253 \mathrm{~m}$

$r_{t}=0.00936 \mathrm{~m}$

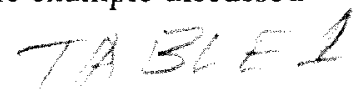


speed of sound in gas/

particle mixture

speed of combustion

products at the burning

surface

Mach number at the

burning surface

$$
\begin{aligned}
& \bar{a}=\sqrt{\left(\bar{\gamma} R T_{c}\right) /\left(1+C_{m}\right)}=1075 \mathrm{~m} / \mathrm{s} \\
& \bar{u}_{b}=\left(\rho_{p} / \rho\right) \bar{r}_{b}=1.86 \mathrm{~m} / \mathrm{s} \\
& \bar{M}_{b}=0.00173
\end{aligned}
$$

\section{Acknowledgments}

The authors are indebted to S. I. Kim and M. Fuchs for their help in completing some of the calculations and to J. N. Levine for providing the numerical code for the nonlinear combustion instability study. This work was partly supported by the California Institute of Technology and Pennsylvania State University.

\section{References}

'Marble, F. E., "Dynamics of a Gas Containing Small Solid Particles," Combustion and Propulsion, 5th AGARD Colloquium, Pergamon, New York, 1963, pp. $175-213$.

${ }^{2}$ Marble, F. E., "The Dynamics of Dusty Gases," Annual Review of Fluid Mechanics, Vol. 2, 1970, pp. 397-446.

${ }^{3}$ Culick, F. E. C., "Stability of Three-Dimensional Motions in a Combustion Chamber," Combustion Science and Technology, Vol. 10, 1975, pp. 109-124.

${ }^{4}$ Prakash, S., and Sirignano, W. A., "Theory of Convective Droplet Vaporization with Unsteady Heat Transfer in the Circulating Liquid Phase," International Journal of Heat and Mass Transfer, Vol. 23, 1980, 253-268.

${ }^{5}$ Rangel, R. H., and Sirignano, W. A., "An Evaluation of the Point-Source Approximation in Spray Calculations," Numerical Heat Transfer, Pt. A, Vol. 16, 1989, pp. 57-57.

${ }^{6}$ Sirignano, W. A., "The Formulation of Spray Combustion Models: Resolution Compared to Droplet Spacing," Journal of Heat Transfer, Vol. 108, 1986, pp. 633639.

${ }^{7}$ Culick, F. E. C., "Stability of Longitudinal Oscillations with Pressure and Velocity Coupling in a Solid Propellant Rocket Motor," Combustion Science and Technology, Vol. 2, 1970, pp. 179-201.

${ }^{8}$ Culick, F. E. C., "The Stability of One-Dimensional Motions in a Rocket Motor," Combustion Science and Technology, Vol. 7, 1973, pp. 165-175.

'Zinn, B. T., and Powell, E. A., "Application of the Galerkin Method in the Solution of Combustion Instability Problems," Proceedings of the 19th International Astronautical Congress, Ottawa, Canada, Vol. 3, 1970, pp. 59-73.

${ }^{10} \mathrm{Zinn}$, B. T., and Powell, E. A., "Nonlinear Instability in Liquid-Propellant Rocket Engines," Thirteenth Symposium (International) on Combustion, The Combustion Inst., Pittsburgh, PA, 1971, pp. 491-503.

${ }^{11}$ Culick, F. E. C., "Nonlinear Behavior of Acoustic Waves in Combustion Chambers-Parts I and II," Acta Astronautica, Vol. 3, 1976, pp. 715-756.

${ }^{12}$ Krylov, N., and Bogoliubov, N., Introduction to Nonlinear Mechanics, Princeton Univ. Press, Princeton, NJ, 1947. 
${ }^{13}$ Yang, V., and Culick, F. E. C., "On the Existence and Stability of Limit Cycles for Transverse Acoustic Oscillations in a Cylindrical Combustion Chamber. 1: Standing Modes," Combustion Science and Technology, Vol. 72, No. 1, 1990, pp. 37-65.

${ }^{14}$ Yang, V., and Culick, F. E. C., "Nonlinear Analysis of Transverse Pressure Oscillations in Ramjet Engines," AIAA Paper 86-0001, 1986.

${ }^{15}$ Yang, V., Hsieh, K. C., and Tseng, I. S., "Velocity-Coupled Flow Oscillations in a Simulated Solid-Propellant Rocket Environment," AIAA Paper 88-0543, 1988.

${ }^{16}$ Lovine, R. L., Dudley, D. P., and Waugh, R. C., "Standardized Stability Prediction Method for Solid Rocket Motors," Vols. I, II, and III, Aerojet Solid Propulsion Co., AFRPL-TR-76-32, 1976.

${ }^{17}$ Nickerson, G. R., Culick, F. E. C., and Dang, L. G., "Standard Stability Prediction Method for Solid Rocket Motors, Axial Mode Computer Program, User's Manual," Software and Engineering Associates, Inc., AFRPL TR-83-017, 1983.

${ }^{18}$ Denison, M. R., and Baum, E., "A Simplified Model of Unstable Burning in Solid Propellants," American Rocket Society Journal, Vol. 31, No. 8, 1961, pp. $1112-1122$.

${ }^{19}$ Culick, F. E. C., "A Review of Calculations for Unsteady Burning of a Solid Propellant," AlAA Journal, Vol. 6, No. 6, 1968, pp. 2241-2255.

${ }^{20}$ Epstein, P. S., and Carhart, R. B., "The Absorption of Sound in Suspensions and Emulsions. I. Water Fog in Air," Journal of the Acoustical Society of America, Vol. 25, 1953, pp. 553-565.

${ }^{21}$ Temkin, S., and Dobbins, R. A., "Attenuation and Dispersion of Sound by Particulate-Relaxation Processes," Journal of the Acoustical Society of America, Vol. 40, No. 2, 1966, pp. 317-324.

${ }^{22}$ Temkin, S., and Dobbins, R. A., "Measurements of Attenuation and Dispersion of Sound by Aerosols," Journal of the Acoustical Society of America, Vol. 40, No. 5, 1966, pp. 1016-1024.

${ }^{23}$ Kraeutle, K. J., Mathes, H. B., and Doherty, G. L., "Combustion Instability Studies Using Metallized Solid Propellants: Additional Evidence for the Validity of Particle Damping Theory," 13th JANNAF Combustion Conference Proceedings, C.P.I.A. Publication 281, Vol. II, 1976, pp. 155-166.

${ }^{24}$ Marble, F. E., "Some Gasdynamic Problems in the Flow of Condensing Vapors," Astronautica Acta, Vol. 14, 1969, pp. 585-614.

${ }^{25}$ Marble, F. E., and Wooten, D. C., "Sound Attenuation in a Condensing Vapor," Physics of Fluids, Vol. 13, 1970, pp. 2657-2664.

${ }^{26}$ Korman, H. F., and Micheli, P. L., "Nonlinear Particulate Damping of Acoustic Oscillations," 8th JANNAF Combustion Conference Proceedings, 1971.

${ }^{27}$ Levine, J. N., Fuchs, M. D., and Park, T. W., "Particle Damping of Nonlinear Longitudinal-Mode Oscillations," AIAA Paper 88-2943, 1988.

${ }^{28}$ Cantrell, R. H., and Hart, R. W., "Interaction Between Sound and Flow in Acoustic Cavities: Mass, Momentum and Energy Considerations," Journal of the Acoustical Society of America, Vol. 36, No. 4, 1964, pp. 697-706.

${ }^{29}$ Flandro. G. A., and Jacobs, H. R., "Vortex-Generated Sound in Cavities," Aeroacoustics: Jet and Combustion Noise, edited by H. T. Nagamatsu, Vol. 37, Progress in Astronautics and Aeronautics, AIAA, New York, 1975.

${ }^{30}$ Culick, F. E. C., and Magiawala, K. R., "Excitation of Acoustic Modes in a Chamber by Vorex Shedding," Journal of Vibration and Sound, Vol. 64, No. 3, 1979, pp. $455-457$. 
${ }^{31}$ Nomoto, H., and Culick, F. E. C., "An Experimental Investigation of Pure Tone Generation by Vortex Shedding in a Duct," Journal of Sound and Vibration, Vol. 84, 1982, pp. 247-252.

${ }^{32}$ Flandro, G. A., and Finlayson, P. A., "Nonlinear Interactions Between Vortices and Acoustic Waves in a Combustion Chamber," 21st JANNAF Combustion Conference Proceedings, 1984, pp. 71-81.

${ }^{33}$ Aaron, K., and Culick, F. E. C., "Coupled Vortex Shedding and Acoustic Resonances in a Duct," 22nd JANNAF Combustion Conference Proceedings, 1985.

${ }^{34}$ Dunlap, R., and Brown, R. S., "Exploratory Experiments on Acoustic Oscillations Driven by Periodic Vortex Shedding," AIAA Journal, Vol. 19, No. 3, 1981, pp. $408-409$.

${ }^{35}$ Schadow, K. C., Gutmark, E., Parr, T. P., Parr, D. M., Wilson, K. J., and Crump, J. H., "Large Scale Coherent Structures as Drivers of Combustion Instability," AIAA Paper 87-1326, 1987.

${ }^{36}$ Flandro, G. A., "Vortex Driving Mechanism in Oscillatory Rocket Flows," Journal of Propulsion and Power, Vol. 2, No. 3, 1986, pp. 206-214.

${ }^{37}$ Shapiro, A. H., The Dynamics and Thermodynamics of Compressible Fluid Flow, Vol. 1, Ronald, New York, 1953.

${ }^{38}$ Krier, H., Tien, J., Sirignano, W. A., and Summerfield, M., "Nonsteady Burning Phenomenon of Solid Propellants: Theory and Experiment," AIAA Journal, Vol. 6, No. 2, 1968, pp. 278-285.

${ }^{39}$ Culick, F. E. C., "Remarks on Entropy Production in the One-Dimensional Approximation to Unsteady Flow in Combustion Chambers," Combustion Science and Technology, Vol. 15, 1977, pp. 93-97.

${ }^{40}$ Baum, J. D. " "Investigation of Flow Turning Phenomenon: Effect of Upstream and Downstream Propagation," AIAA Paper 88-0544, 1988.

${ }^{41}$ Magiawala, K., and Culick, F. E. C., "Measurements of Energy Exchange Between Acoustic Fields and Non-Uniform Steady Fields," Journal of Vibration and Sound, Vol. 75, 1979, pp. 503-512.

${ }^{42}$ Van Moorhem, W. K., "Flow-Turning in Solid-Propellant Rocket Combustion Stability Analysis," AlAA Journal, Vol. 20, No. 10, 1982, pp. 1420-1425.

${ }^{43}$ Derr, R. L., and Mathes, H. B., "Cold Gas Acoustic Tests for Resolving T-Burner Uncertainties," Proceedings of the 11th JANNAF Combustion Meeting, 1974.

${ }^{44}$ Mathes, H. B., and Price, E. W., "Methods for Determining Characteristics of Acoustic Waves in Rocket Motors," Journal of Spacecraft and Rockets, Vol. 12, No. 1, 1975, pp. 39-43.

${ }^{45}$ Culick, F. E. C. " "T-Burner Testing of Metallized Solid Propellants," Air Force Rocket Propulsion Laboratory, Rept. AFRPL-TR-74-28, 1974.

${ }^{46}$ Paparizos, L., and Culick, F. E. C., "The Two-Mode Approximation to Nonlinear Acoustics in Combustion Chambers. I. Exact Solution for Second Order Acoustics," Combustion Science and Technology, Vol. 65, No. 1-3, 1989, p. 39.

${ }^{47}$ Awad, E., and Culick, F. E. C., "On the Existence and Stability of Limit Cycles for Longitudinal Acoustic Modes in a Combustion Chamber," Combustion Science and Technology, Vol. 46, 1986, pp. 195-222.

${ }^{48}$ Levine, J. N., and Culick, F. E. C., "Numerical Analysis of Nonlinear Longitudinal Combustion Instability in Metalized Solid-Propellant Rocket Motors, Vol. 1 Analysis and Results," Ultrasystems, Inc., AFRPL TR-72-88, 1972. 
${ }^{49}$ Levine, J. N., and Culick, F. E. C., "Nonlinear Analysis of Solid Rocket Combustion Instability," Ultrasystems, Inc., AFRPL TR-74-45, 1974.

${ }^{50}$ Kooker, D. E., "Numerical Solution of Axial-Mode Instability Problems in Solid-Propellant Rocket Motors," Ballistic Research Laboratory Contract Rept. 141,1974

${ }^{51}$ Baum, J. D., and Levine, J. N., "Numerical Techniques for Solving Nonlinear Instability Problems in Solid Rocket Motors," AIAA Journal, Vol. 20, No. 7, 1982, pp. $955-961$.

${ }^{52}$ Harten, A., and Zwas, G., "Self-Adjusting Hybrid Schemes for Shock Computations," Journal of Computational Physics, Vol. 9, 1972, pp. 508-583.

${ }^{53}$ Harten, A., "The Artificial Compression Method for Computation of Shocks and Contact Discontinuities: III. Self-Adjusting Hybrid Schemes," AFOSR TR77-0659, 1977.

${ }^{54}$ Baum, J. D., Lovine, R. L., and Levine, J. N., "Pulsing Techniques for SolidPropellant Rocket Motors: Modeling and Cold-Flow Testing," Journal of Spacecraft and Rockets, Vol. 20, No. 2, 1983, pp. 150-157.

${ }^{55}$ Lovine, R. L., Baum, J. D., and Levine, J. N., "Ejecta Pulsing of Subseale Solid Propellant Rocket Motors," AIAA Journal, Vol. 23, No. 3, 1985, pp. 416423.

${ }^{56}$ Baum, J. D., Levine, J. N., and Lovine, R. L., "Pulsed Instability in Rocket Motors: A Comparison Between Predictions and Experiments," Journal of Propulsion and Power, Vol. 4, No. 4, 1988, pp. 308-316.

${ }^{57}$ Sabnis, J. S., Giebeling, H. J., and McDonald, H., "Navier-Stokes Analysis of Solid Propellant Rocket Motor Internal Flows," Journal of Propulsion and Power, Vol. 5, No. 6, 1989, pp. 657-664.

${ }^{58}$ Hsieh, K. C., Yang, V., and Tseng, I. S., "Navier-Stokes Calculations of SolidPropellant Rocket Motor Internal Flowfields," AIAA Paper 88-3181, 1988.

${ }^{59}$ Tsien, H. S., "The Transfer Functions of Rocket Nozzles," Journal of the American Rocket Society, Vol. 22, 1952, pp. 139-143.

${ }^{\infty}$ Crocco, L., and Cheng, S.-I., Theory of Combustion Instability in Liquid Propellant Rockets, AGARDograph No. 8, Butterworths, London, 1956.

${ }^{\circ}$ Flandro, G. A., "Solid Propellant Acoustic Admittance Correction," Journal of Sound and Vibration, Vol. 36, No. 3, 1974, pp. 297-312.

${ }^{62}$ Vuillot, F., and Avalon, G., "Acoustic Boundary Layers in Solid Propellant Rocket Motors Using Navier-Stokes Equations," Journal of Propulsion and Power, Vol. 7, No. 2, 1991, pp. 231-239.

${ }^{63}$ Tseng, I., and Yang, V., "Interactions of Acoustic Waves and Combustion of Homogeneous Propellant in Rocket Motors," AIAA Paper 92-0101, 1992. 